\title{
The Strict $\omega$-Groupoid Interpretation of Type Theory
}

\author{
Michael A. Warren
}

\begin{abstract}
Hofmann and Streicher showed that there is a model of the intensional form of Martin-Löf's type theory obtained by interpreting closed types as groupoids. We show that there is also a model when closed types are interpreted as strict $\omega$-groupoids. The nonderivability of various truncation and uniqueness principles in intensional type theory is then an immediate consequence. In the process of constructing the interpretation we develop some $\omega$-categorical machinery including a version of the Grothendieck construction for strict $\omega$-categories.
\end{abstract}

\section{Introduction}

In [9], Hofmann and Streicher established the existence of a model of the intensional form of Martin-Löf's type theory [16] using the category Gpd of small groupoids. This seminal result - and the related observation that the identity types in this theory form a kind of weak groupoid - is the starting point for much of the subsequent research relating category theory, homotopy theory and type theory. Type theoretically, the importance of the groupoid model is that it establishes the nonderivability of certain type theoretic principles relating to the identity types.

Recall that in Martin-Löf's type theory if $A$ is a type and $a, b$ are terms of type $A$, then there exists a type $\underline{A}(a, b)$ called the identity types of $A$ (from a to $b)$. Under the Curry-Howard correspondence $\underline{A}(a, b)$ may be thought of as the proposition which asserts that $a$ and $b$ are equal. The distinguishing feature of the intensional form of the theory from the extensional form is the absence in the former of the reflection rule which states that if there exists a term of type $\underline{A}(a, b)$, then $a=b$, where this latter equality is the primitive equality relation of the theory. Thus, identity types are trivial in the extensional theory. Prior to [9] it was unknown whether certain principles of a similar character to the reflection rule were in fact derivable. For example, it was unknown whether there could be more than one distinct "identity proof." I.e., whether $f=g$ when $f, g: \underline{A}(a, b)$. This principle is referred to as uniqueness of identity proofs in the literature and it is refuted in the groupoid model. (Note that in ibid. the principle of uniqueness of identity proofs really only refers to uniqueness up to propositional equality, but the groupoid model refutes both of these principles.) For in the groupoid model, a

2010 Mathematics Subject Classification. 03B15, 18D05.

This is the final form of the paper. 
(closed) type $A$ is interpreted as a groupoid $\llbracket A \rrbracket$, a term $a: A$ is interpreted as an object of $\llbracket A \rrbracket$ and the identity type $\underline{A}(a, b)$ is interpreted as the (discrete) groupoid consisting of all arrows $\llbracket a \rrbracket \rightarrow \llbracket b \rrbracket$ in $\llbracket A \rrbracket$. Thus, any groupoid $G$ containing a pair of distinct parallel arrows refutes the uniqueness of identity proofs.

Although the groupoid model is useful for refuting certain type theoretic principles such as uniqueness of identity proofs, it fails to adequately exhibit higherdimensional structure of the identity types. E.g., although the reflection rule is refuted the corresponding rule which asserts that all identity types of identity types are trivial is valid in the model since identity types are interpreted as discrete groupoids. I.e., the rule

$$
\frac{p: \underline{\underline{A}}(a, b)(f, g)}{f=g: \underline{A}(a, b)}
$$

is valid. At the time Hofmann and Streicher conjectured that it should be possible to obtain models which would refute such "truncation principles" by interpreting types as some kind of higher-dimensional groupoids. It is the aim of this paper to show that this is indeed the case. In particular, we construct a model of intensional type theory which extends the original groupoid model by using strict $\omega$-groupoids. As such, this is the first known model of intensional type theory (aside from the syntactic model) which is truly higher-dimensional. In the terminology of [1] the model refutes all of the truncation rules $\mathrm{TR}_{n}$.

The interpretation of type theory using $\omega$-groupoids directly extends the original groupoid interpretation. Contexts $\Gamma$ are interpreted as small $\omega$-groupoids $\llbracket \Gamma \rrbracket$ and types $\Gamma \vdash A$ in context are interpreted as morphisms (of $\omega$-categories) $\llbracket \Gamma \rrbracket \rightarrow \omega$-Gpd. A term $\Gamma \vdash a: A$ is interpreted as a section of the projection $\int \llbracket A \rrbracket \rightarrow \llbracket \Gamma \rrbracket$ where $\int \llbracket A \rrbracket$ denotes the ( $\omega$-categorical version of the) Grothendieck construction of $\llbracket A \rrbracket$. For a closed type $A$, the identity type $\underline{A}(a, b)$ is then interpreted as the $\omega$-groupoid which has as objects arrows $\llbracket a \rrbracket \rightarrow \llbracket b \rrbracket$ in $\llbracket A \rrbracket$. As in the groupoid model, the dependent sums and products are given by left- and right-adjoints, respectively, to suitable reindexing functors.

Relation to other work. There has been much recent work on relating Martin-Löf's type theory to homotopy theory and higher-dimensional category theory and we will now briefly summarize the relation of the current paper to other such work. First, following a suggestion by Moerdijk, it was shown in [2] that intensional type theory (at least the fragment containing identity types and dependent sums) can be interpreted in weak factorization systems and Quillen's model categories which satisfy certain coherence conditions. It was then shown by Gambino and Garner [5] that there is a weak factorization system in the category of contexts. It was also shown, by van den Berg and Garner [25] and independently by Lumsdaine [15], that the identity type construction gives rise to weak $\omega$-groupoids in the sense of Batanin and Leinster [3,14]. Garner [6] has also introduced a "two-dimensional" type theory which is a form of intensional type theory and he has proved that it is sound and complete with respect to a semantics valued in the 2-categories (as such, these theories necessarily satisfy the corresponding truncation rule). In [1] the homotopy theory of the category of groupoids and a fortiori homotopy 1-types is related to the homotopy theory of the 1-truncated form of intensional type theory.

We would also like to mention the recent work of Lafont, Métayer and Worytkiewicz [12] in which they describe a model structure on the category of strict 
$\omega$-categories which corresponds to the "natural" or "folk" model structure on the category of small categories. Ultimately we would like to be able to relate their work to the model constructed here. In particular, one should be able to use exponentiation by the $\omega$-groupoid interval $\mathbf{I}$ - which is the co- $\omega$-groupoid object in $\omega$-Cat with exactly two 0-cells, two invertible 1-cells between them, two invertable 2-cells between those, and so forth - to interpret identity types. (In the setting of ordinary 1-dimensional groupoids this kind of construction has been studied in [27] and related results on cocategory objects were obtained in [28].) Exponentiation by $\mathbf{I}$ has been studied by Métayer [17] and is used in [12] for the construction of path objects in the model structure. In order to develop such an approach using $\mathbf{I}$ it appears to be necessary to generalize also the notion of split fibration to higherdimensions. The notion of Grothendieck 2-fibration has been studied by Hermida [7] and, for 2-groupoids, by Moerdijk and Svennson [18]. However, we are unaware of similar work in higher dimensions.

Summary of the paper. In Section 2 we recall some basic facts and definitions concerning $\omega$-categories. In Section 3 we extend the familiar Grothendieck construction to the setting of $\omega$-categories and functors $A: \mathcal{C} \rightarrow \omega$-Cat. This construction is crucial since it allows us to interpret context extension and it provides the data necessary for the interpretation of open terms. In Section 4 we introduce the notion of $\omega$-groupoid with which we will be concerned. We also describe a "dual" version of the Grothendieck construction. For $\omega$-groupoid valued functors there is an induced functor $\neg$ relating the Grothendieck construction and its dual which we will require in order to interpret identity types and dependent products. In Section 5 we finally describe the interpretation of identity types. For closed types $A$ and terms $a$ and $b$ of type $A$ the identity type $\underline{A}(a, b)$ is easily described as the $\omega$-groupoid which has as objects arrows $a \rightarrow b$ and with $(n+1)$-cells given by $(n+2)$-cells of $A$ bounded in dimension 0 by $a$ and $b$. However, in order to most efficiently prove the soundness of the elimination rule for identity types it is necessary to give an interpretation of the corresponding open identity type. This is essentially a fibred version of the foregoing type, but is considerably more involved in the case of $\omega$-groupoids than it was for groupoids. Section 6 is concerned with the proof of the soundness of the introduction and elimination rules for identity types. Finally, the main results of the paper (the soundness theorem and its consequences) are collected in Section 7. Appendix A contains a summary of the rules governing identity types, dependent products, and dependent sums in the theory considered in this paper. Appendix B contains a brief description of the comma $\omega$ categories featuring in the description of the universal property of the Grothendieck construction and the proof that the Grothendieck construction does indeed possess the correct universal property.

It is worth mentioning that we assume familiarity on the part of the reader with Martin-Löf's type theory and we refer the reader to $[4,8,10,16,19,23,24]$ for further details. There is also a brief survey of intensional type theory contained in [1] which includes a summary of the kinds of truncation principles which are refuted by the model.

Acknowledgements. The results of the present paper originally occur in the author's Ph.D. thesis [27] and I thank my thesis supervisor Steve Awodey for numerous insightful comments and discussions of this work. I also thank the other 
members of my thesis committee - Nicola Gambino, Alex Simpson and Thomas Streicher - for discussion of and comments on this work. The anonymous referee also provided a number of useful comments and suggestions. Finally, I thank Pieter Hofstra, Peter LeFanu Lumsdaine and Phil Scott for useful conversations regarding this material.

\section{Generalities on $\omega$-categories}

In this section we recall the definition of strict $\omega$-categories and $\omega$-groupoids, fix notation and recall several useful facts about these structures. We refer the reader to $[14,21,22,26]$ for further details regarding the theory of strict $\omega$-categories.

2.1. $\omega$-categories, functors and transformations. Just as categories are (directed) reflexive graphs equipped with the additional structure given by identities and composition, so too $n$-categories are reflexive $n$-globular sets with additional structure and $\omega$-categories are reflexive globular sets with additional structure. Here we recall that reflexive globular sets $A$ are the (unbounded) higher-dimensional analogues of reflexive graphs and, as such, consists of sets $A_{n}$ graded by nonnegative integers $n$ together with source, target and identity functions

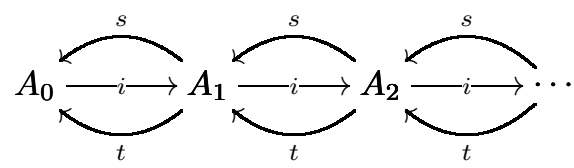

such that the globular identities

$$
s \circ s=s \circ t, \quad t \circ t=t \circ s,
$$

and the equations

$$
s \circ i=1_{A_{n}}=t \circ i
$$

are satisfied. A (strict) $\omega$-category consists of a reflexive globular set $A$ and, for all $p \geq 0$ and $n>0$, composition functions

$$
A_{n} \times_{A_{p}} A_{n} \stackrel{*_{p}}{\longrightarrow} A_{n},
$$

where $A_{n} \times_{A_{p}} A_{n}$ is the pullback

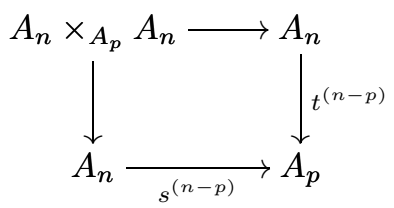

with

$$
l^{k}:=\underbrace{l \circ l \circ \cdots \circ l}_{k \text {-times }},
$$

for any $k \geq 0$ and $l=s, t$. This data is required to satisfy the following conditions:

\section{Domain and Codomain Laws.}

$$
l\left(g *_{p} f\right)= \begin{cases}l(g) *_{p} l(f) & \text { if } p<(n-1) \\ = \begin{cases}s(f) & \text { if } l=s \\ t(g) & \text { if } l=t\end{cases} & \text { if } p=(n-1) .\end{cases}
$$

for $l=s, t$. 
Associativity Laws. Each operation $*_{p}$ is associative.

Unit Laws. Given $f$ in $A_{n}$,

$$
i^{(n-p)}\left(t^{(n-p)}(f)\right) *_{p} f=f=f *_{p} i^{(n-p)}\left(s^{(n-p)}(f)\right) .
$$

Interchange Laws. Given $q<p<n$ and $f, g, h, k$ in $A_{n}$ such that the composites $\left(y *_{q} x\right),\left(k *_{q} h\right),\left(h *_{p} f\right)$ and $\left(k *_{p} g\right)$ are defined,

$$
\left(k *_{p} g\right) *_{q}\left(h *_{p} f\right)=\left(k *_{q} h\right) *_{p}\left(g *_{q} f\right),
$$

and

$$
i(g) *_{q} i(f)=i\left(g *_{q} f\right) .
$$

Sometimes we refer to composition $*_{0}$ along 0 -cells as horizontal composition. Given two $n$-cells $f$ and $g$, if $s(f)=s(g)$ and $t(f)=t(g)$, then we say that $f$ and $g$ are parallel (to one another). For example, if $f$ and $g$ are $n$-cells such that $\left(g *_{p} f\right)$ is defined, then $s^{(n-p)}(f), s^{(n-p)}(g), t^{(n-p)}(f)$ and $t^{(n-p)}(g)$ are all parallel. When no confusion will result we often omit mention of identity maps. E.g., if $f: x \rightarrow y$ is a 1-cell and $\alpha: g \Rightarrow h$ is a 2-cell with $s(g)=y$, we denote $\alpha *_{0} i(f)$ by $\alpha *_{0} f$.

A (strict $\omega$-)functor $F: \mathcal{C} \rightarrow \mathcal{B}$ between $\omega$-categories is a map of globular sets which preserves all composition and identities. We often refer to $\omega$-functors simply as functors when it is understood that we are dealing with $\omega$-categories. The category of small $\omega$-categories and functors between them is denoted by $\omega$-Cat. Just as the category Cat of small categories and functors between them is monadic over the category of directed graphs, so too $\omega$-Cat is monadic over the category of globular sets (cf. [14] for an explicit description of the monad). Indeed, $\omega$-Cat is a Cartesian closed category with all (small) limits and colimits. Henceforth we often denote $\omega$-categories by $\mathcal{C}, \mathcal{B}, \ldots$ Clearly every $\omega$-category is also an $n$-category, for $1 \leq n$ and similarly for $\omega$-functors.

Given functors $F, G: \mathcal{C} \rightrightarrows \mathcal{B}$ between $\omega$-categories $\mathcal{C}$ and $\mathcal{B}$, a natural transformation $\alpha: F \Rightarrow G$ consists of an assignment of 1-cells $\alpha_{x}: F x \rightarrow G x$ for objects $x$ of $\mathcal{C}$ such that the following (somewhat schematic) diagram commutes:

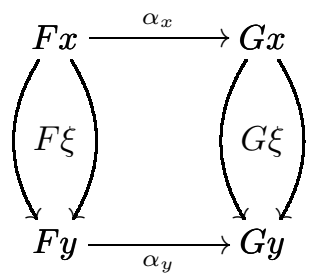

for every $k$-cell $\xi$ bounded by 0 -cells $x$ and $y$ (see Section 2.2 for an explanation of the notation employed here). I.e., if $\xi$ is any $k$-cell, for $k \geq 1$, such that $s^{k} \xi=x$ and $t^{k} \xi=y$, then

$$
\alpha_{y} *_{0} F \xi=G \xi *_{0} \alpha_{x} .
$$

Passing up one dimension, suppose we are given functors $F$ and $G$ as above together with natural transformations $\alpha$ and $\beta$ from $F$ to $G$. Then, a modification or 2transformation $\varphi: \alpha \Rightarrow \beta$ consists of an assignment of 2-cells $\varphi_{x}: \alpha_{x} \rightarrow \beta_{x}$ of $\mathcal{B}$ parameterized by objects $x$ of $\mathcal{C}$ subject to the condition that, for any arrow 
$f: x \rightarrow y$ of $\mathcal{C}$, the following diagram commutes:

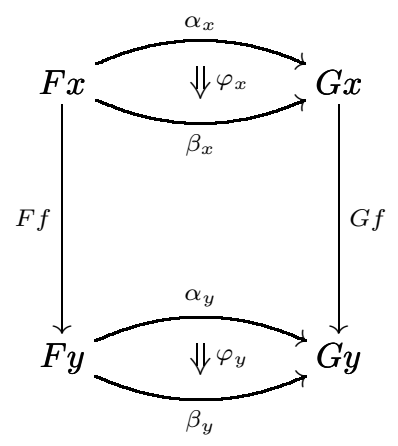

I.e.,

$$
\varphi_{y} *_{0} F f=G f *_{0} \varphi_{x}
$$

for $f: x \rightarrow y$ an arrow of $\mathcal{C}$.

It is possible to generalize inductively to higher-dimensional transformations. In particular, assuming we have defined $n$-transformations, for $n \geq 2$, in such a way that the obvious boundary conditions are satisfied a $(n+1)$-transformation $\psi$ from an $n$-transformation $\gamma$ to a $n$-transformation $\delta$ consists of a family of $n$-cells $\psi_{x}: \gamma_{x} \Rightarrow \delta_{x}$ in $\mathcal{B}$ parameterized by objects $x$ of $\mathcal{C}$ such that, whenever $f: x \rightarrow y$ is an arrow in $\mathcal{C}$, the naturality condition

$$
\psi_{y} *_{0} F f=G f *_{0} \psi_{x}
$$

is satisfied. With these definitions it is straightforward to verify that the following more general naturality conditions are also satisfied:

Lemma 2.1. If $\xi$ is a $k$-cell of $\mathcal{C}$ bounded by 1-cells $f, g: x \rightrightarrows y$ and $\varphi$ is a $(n+1)$-transformation bounded by functors $F, G: \mathcal{C} \rightarrow \mathcal{B}$, then

$$
\varphi_{y} *_{0} F \xi=G \xi *_{0} \varphi_{x}
$$

With $\omega$-functors and these higher-dimensional transformations $\omega$-Cat itself exhibits the combinatorial structure of an $\omega$-category.

Proposition 2.2. The category $\omega$-Cat is a large $\omega$-category with $(n+1)$-cells given by $n$-transformations.

2.2. Notation and conventions. It will be convenient to introduce some conventions governing diagrams in higher dimension. In particular, we will often want to describe the various boundaries of $m$-cells $\varphi$ in $\omega$-categories. In particular, we may wish to indicate diagrammatically the $n$-cells bounding such a $\varphi$ even when $m>n+2$ so that drawing the details of the diagram would be cumbersome. As such, we will instead often include diagrams such as the following:

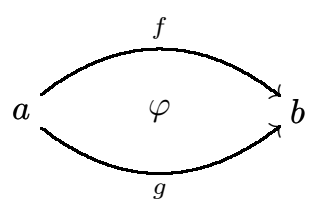


where $a$ and $b$ are $n$-cells and $\varphi$ is an $m$-cell for $m>n+2$. Such diagrams are oriented from "top-to-bottom" unless otherwise stated. I.e., the diagram indicates that

$$
s^{m-n-1}(\varphi)=f \quad \text { and } \quad t^{m-n-1}(\varphi)=g .
$$

In the few cases where there is no "top-to-bottom" option available, the cells should be oriented "left-to-right." In this section, and throughout this chapter, we will often be dealing with "hom- $\omega$-categories" of the form $\mathcal{C}(a, b)$ where $\mathcal{C}$ is an $\omega$-category and $a, b$ are parallel cells of $\mathcal{C}$. In this setting, or similar ones, the index of a composition $\left(\gamma *_{n} \delta\right)$ always refers to the dimension in $\mathcal{C}$ and not in $\mathcal{C}(a, b)$.

There exists an endofunctor $(-)^{+}$on $\omega$-Cat called the dimension shift functor which shifts the dimension of an $\omega$-category. Specifically, given an $\omega$-category $\mathcal{C}$, $\mathcal{C}^{+}$has as objects 1-cells of $\mathcal{C}$ and, in general, $n$-cells of $\mathcal{C}^{+}$are $(n+1)$-cells of $\mathcal{C}$. Similarly, given $\omega$-category $\mathcal{C}$ and objects $x$ and $y$ of $\mathcal{C}$, the hom set $\mathcal{C}(x, y)$ can be made into an $\omega$-category - which we sometimes denote by $\mathcal{C}_{1}(x, y)$ to emphasize the dimension - by defining 0-cells to be arrows $f: x \rightarrow y$ and $(n+1)$-cells to be $n$-cells in the obvious way. Similarly, given parallel $(n+1)$-cells $f, g$ of $\mathcal{C}$, there is an $\omega$-category $\mathcal{C}_{n+2}(f, g)$ which has 0 -cells $(n+2)$-cells $\alpha: f \Rightarrow g$ and so forth. For parallel $n$-cells $f$ and $g$ with $n \geq 1$, there exists an inclusion functor

$$
\mathcal{C}_{(n+1)}(f, g) \rightarrow\left(\mathcal{C}_{n}(s f, t g)\right)^{+}
$$

which sends a $(n+1)$-cell $\alpha: f \rightarrow g$ to itself, and similarly for higher-dimensional cells. We will occasionally make use of such functors without giving them an explicit name.

It will also be convenient to fix notation for dealing with functors $A: \mathcal{C} \rightarrow$ $\omega$-Cat. Given a small $\omega$-category $\mathcal{C}$ and a functor $A: \mathcal{C} \rightarrow \omega$-Cat we denote by $A_{x}$ the category obtained by applying $A$ to an object $x$ of $\mathcal{C}$ and, when $f: x \rightarrow y$ is an arrow in $\mathcal{C}, A_{f}: A_{x} \rightarrow A_{y}$ denotes the induced functor. We employ similar notation for higher-dimensional cells. This convention will later allow us to avoid excessive use of parentheses. When $z$ is any 0 -cell of $A_{x}$ we denote $A_{f}(z)$ by $(z . f)$. Similarly, by definition $A_{\gamma}$, for $\gamma$ a $(n+1)$-cell with $n \geq 1$, is given by a family of $n$-cells parameterized by 0 -cells of its domain category (say) $A_{x}$ and we denote by $(z \cdot \gamma)$ the $n$-cell $\left(A_{\gamma}\right)_{z}$. In many of the constructions given below we will deal with $\omega$-categories having as their cells tuples of cells of other $\omega$-categories. Such tuples will often be abbreviated using "vector" notation $\vec{f}$.

\section{The $\omega$-categorical Grothendieck construction}

When dealing with categories, there is, for each $\mathcal{C}$, a monadic adjunction

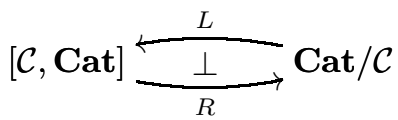

of 2-categories where the left-adjoint is given by the comma category

$$
L(F)=(F \downarrow-),
$$

for $F: \mathcal{D} \rightarrow \mathcal{C}$, and the right-adjoint is given by taking the projection from the Grothendieck construction

$$
R(G)=\int G \stackrel{\pi}{\longrightarrow} \mathcal{C}
$$


for $G: \mathcal{C} \rightarrow$ Cat. An analogous situation occurs in the case of $\omega$-categories and, in particular, the 2 -category $[\mathcal{C}, \omega$-Cat $]$ is also monadic over $\omega$-Cat $/ \mathcal{C}$. The rightadjoint is again given by an $\omega$-categorical version of the Grothendieck construction. This construction will feature prominently in the interpretation of type theory since it allows us to interpret the extension of contexts. As in the case of Cat, the Grothendieck construction $\int A$ of a functor $A: \mathcal{C} \rightarrow \omega$-Cat (or category of elements of $A$ ) can be described (in terms of its universal property) as the coend

$$
\int^{x}(x \downarrow \mathcal{C}) \times A_{x}
$$

which exists since $\omega$-Cat is bicomplete. Here $(x \downarrow \mathcal{C})$, like the comma construction which gives the left-adjoint $L$, is a lax version of the usual comma category. I.e., instead of having as 1-cells commutative triangles, an arrow from an object $g: x \rightarrow y$ to $h: x \rightarrow z$ is a pair $\left(f, f^{\prime}\right)$ such that $f: y \rightarrow z$ and

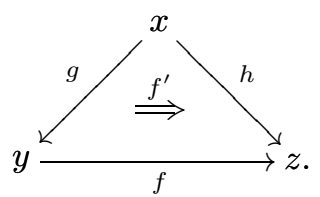

The higher-dimensional cells of this $\omega$-category will not be necessary for our purposes and are described in Appendix B. Although (3.1) is a convenient description of the Grothendieck construction of $A$, it will be useful for our purposes to have a direct (combinatorial) description of this $\omega$-category. It is to this matter which we now turn. (The verification that the combinatorial description given below of the Grothendieck construction satisfies the universal property of the coend (3.1) is contained in Appendix B.)

3.1. Combinatorial description of the Grothendieck construction. We now give a direct description of the $\omega$-category $\int A$. We leave the proof that the data given below constitutes an $\omega$-category to the reader (the proof can also be found in [27]). In the first two dimensions $\int A$ is the familiar Grothendieck construction of $A$.

0-Cells. The 0-cells of $\int A$ are pairs $\left(x, x_{-}\right)$such that $x$ is an object of $\mathcal{C}$ and $x_{-}$is an object of $A_{x}$.

1-Cells. The 1-cells $\left(x, x_{-}\right) \rightarrow\left(y, y_{-}\right)$are pairs $\left(f, f_{-}\right)$consisting of a 1-cell $f: x \rightarrow y$ in $\mathcal{C}$ and a 1-cell $f_{-}:\left(x_{-} . f\right) \rightarrow y_{-}$in $A_{y}$.

Already at this low dimension there are several features of the definition which should be emphasized. First, in order to define the component $f_{-}$of arrows in $\int A$ we have made a choice of "weight" or "orientation." Namely, we have determined that the codomain of $f_{-}$should be $y_{-}$where we could have as easily determined that its domain should be this object of $A_{y}$. Secondly, fixing objects $\vec{x}$ and $\vec{y}$ of $\int A$, there exists a functor

$$
\mathcal{C}_{1}(x, y) \stackrel{\mathfrak{d}_{\vec{x}, \vec{y}}^{1}}{\longrightarrow} A_{y}
$$

defined by

$$
\mathfrak{d}_{\vec{x}, \vec{y}}^{1}(\gamma):=\left(x_{-} \cdot \gamma\right),
$$

for any $m$-cell $\gamma$ of $\mathcal{C}(x, y)$. Although this functor depends on $\vec{x}$ and $\vec{y}$ we often write $\mathfrak{d}^{1}$ when no confusion will result. With this definition we observe that an 
arrow $\vec{f}: \vec{x} \rightarrow \vec{y}$ has

$$
s\left(f_{-}\right)=\mathfrak{d}^{1}(f) .
$$

In this situation, the object $\mathfrak{d}^{1}(f)$ is said to be the weighted face of $f_{-}$. We will see that the higher-dimensional cells resulting from the construction of $\int A$ also possess suitably "weighted" faces.

2-Cells. Given 1-cells $\vec{f}$ and $\vec{g}$ with common source and target $\vec{x}$ and $\vec{y}$, respectively, a 2-cell $\vec{f} \rightarrow \vec{g}$ consists of $\alpha: f \Rightarrow g$ in $\mathcal{C}$ together with a 2-cell $\alpha_{-}$of $A_{y}$ as indicated in the following diagram:

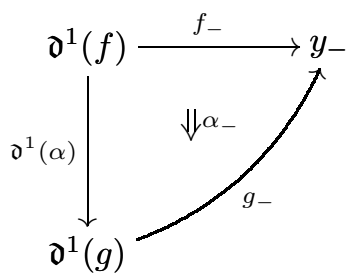

Now, holding $\vec{f}$ and $\vec{g}$ fixed, there exists a functor

$$
\mathcal{C}_{2}(f, g) \stackrel{\mathfrak{d}_{\vec{f}, \vec{g}}^{2}}{\longrightarrow}\left(A_{y}\right)_{1}\left(\mathfrak{d}_{\vec{x}, \vec{y}}^{1}(f), y_{-}\right)
$$

defined by

$$
\mathfrak{d}_{\vec{f}, \vec{g}}^{2}(\gamma):=g_{-} *_{0} \mathfrak{d}_{\vec{x}, \vec{y}}^{1}(\gamma)
$$

where $\gamma$ is a $n$-cell of $\mathcal{C}_{2}(f, g)$. Note that in this case $\mathfrak{d}^{1}(\gamma)$ is a $(n+1)$-cell of $A_{y}$ so that this definition makes sense. It is straightforward to verify that this is functorial. Note that an arrow $\vec{\alpha}: \vec{f} \rightarrow \vec{g}$ has

$$
t\left(\alpha_{-}\right)=\mathfrak{d}_{\vec{f}, \vec{g}}^{2}(\alpha) .
$$

As above, $\mathfrak{d}^{2}(\alpha)$ is the weighted face of $\alpha_{-}$. In general, we will see that $(n+1)$-cells of $\int A$ are given by pairs $\left(\varphi, \varphi_{-}\right)$and that each component $\varphi_{-}$comes equipped with a weighted face. Namely, the weighted face of $\varphi_{-}$is $s\left(\varphi_{-}\right)$if $(n+1)$ is even and it is $t\left(\varphi_{-}\right)$if $(n+1)$ is odd. At each stage $n$ we will construct, along with the definition of the $n$-cells, a functor $\mathfrak{d}^{n}(-)$ which gives an explicit description of the weighted faces of $n$-cells.

In general, $\left(\int A\right)_{n}$ is defined by induction on $n$ alternating between even and odd steps in such a way that the following conditions are satisfied:

(1) At each stage $n$ an element of $\left(\int A\right)_{n}$ is a pair $\left(f, f_{-}\right)$such that $f$ is an $n$-cell of $\mathcal{C}$ and $f_{-}$is an $n$-cell of $A_{y}$.

(2) At each stage $(n+1)$, for $n \geq 1$, there is also constructed, for each pair $\vec{\alpha}, \vec{\beta}$ of parallel $n$-cells with source $\vec{f}$ and target $\vec{g}$, a functor $\mathfrak{d}_{\vec{\alpha}, \vec{\beta}}^{n+1}$ such that

$$
\mathcal{C}_{n+1}(\alpha, \beta) \stackrel{\mathfrak{d}_{\vec{\alpha}, \vec{\beta}}^{n+1}}{\longrightarrow}\left(A_{y}\right)_{n}\left(\mathfrak{d}_{\vec{f}, \vec{g}}^{n}(\alpha), g_{-}\right)
$$

if $(n+1)$ is even and

$$
\mathcal{C}_{n+1}(\alpha, \beta) \stackrel{\mathfrak{d}_{\vec{\alpha}, \vec{\beta}}^{n+1}}{\longrightarrow}\left(A_{y}\right)_{n}\left(f_{-}, \mathfrak{d}_{\vec{f}, \vec{g}}^{n}(\beta)\right)
$$

if $(n+1)$ is odd. The functor $\mathfrak{d}^{n+1}$ is called the weighted face functor (in dimension $(n+1)$ determined by $\vec{\alpha}$ and $\vec{\beta})$. 
(3) At each stage $(n+1)$, for $n \geq 0$, it is required that the following weighted face conditions are satisfied:

$$
s\left(\varphi_{-}\right)= \begin{cases}\alpha_{-} & \text {if }(n+1) \text { is even } \\ \mathfrak{d}_{\vec{\alpha}, \vec{\beta}}^{n+1}(\varphi) & \text { if }(n+1) \text { is odd }\end{cases}
$$

and

$$
t\left(\varphi_{-}\right)= \begin{cases}\mathfrak{d}_{\vec{\alpha}, \vec{\beta}}^{n+1}(\varphi) & \text { if }(n+1) \text { is even, } \\ \beta_{-} & \text {if }(n+1) \text { is odd },\end{cases}
$$

when $\vec{\varphi}$ is an $(n+1)$-cell $\vec{\varphi}: \vec{\alpha} \rightarrow \vec{\beta}$.

By the discussion above, the base cases $n=0,1,2$ satisfy these conditions. We now consider the induction steps.

$(\boldsymbol{n}+\mathbf{1})$ is odd. Fix parallel $n$-cells $\vec{f}$ and $\vec{g}$ of $\int A$. A $(n+1)$-cell $\vec{f} \Rightarrow \vec{g}$ consists of a pair $\left(\alpha, \alpha_{-}\right)$with $\alpha: f \Rightarrow g$ an $(n+1)$-cell in $\mathcal{C}$ and $\alpha_{-}$is a $(n+1)$-cell of $A_{y}$ subject to conditions which we will now describe. Let $\vec{v}=s(\vec{f})$ and $\vec{w}=t(\vec{f})$. Then $\vec{v}$ and $\vec{w}$ are $(n-1)$-cells of $\int A$ and therefore, by the induction hypothesis, there exists a weighted face functor $\mathfrak{d}_{\vec{v}, \vec{w}}^{n}: \mathcal{C}_{n}(v, w) \rightarrow\left(A_{y}\right)_{n-1}\left(\mathfrak{d}^{n-1}(v), w_{-}\right)$. As such, $\alpha_{-}$is required to be a $(n+1)$-cell of $A_{y}$ as indicated in the following diagram:

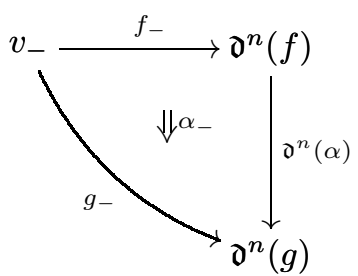

For the weighted functor, we hold $\vec{f}$ and $\vec{g}$ fixed and define

$$
\mathfrak{d}_{\vec{f}, \vec{g}}^{(n+1)}(\gamma):=\mathfrak{d}^{n}(\gamma) *_{(n-1)} f_{-},
$$

for $\gamma$ a $m$-cell of $\mathcal{C}_{(n+1)}(f, g)$. The weighted face conditions are then trivially satisfied.

$(\boldsymbol{n}+\mathbf{1})$ is even. Given parallel $n$-cells $\vec{f}$ and $\vec{g}$ of $\int A$, a $(n+1)$-cell $\vec{f} \Rightarrow \vec{g}$ consists, as above, of a pair $\left(\alpha, \alpha_{-}\right)$with $\alpha: f \Rightarrow g$ in $\mathcal{C}$ and $\alpha_{-}$a $(n+1)$-cell of $A_{y}$ as indicated in the following diagram:

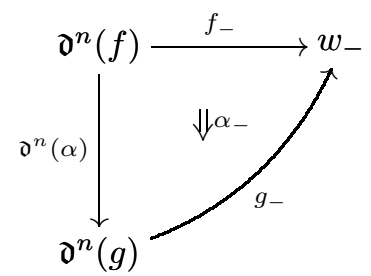

Finally, we define:

$$
\mathfrak{d}_{\vec{f}, \vec{g}}^{(n+1)}(\gamma):=g_{-} *(n-1) \mathfrak{d}^{n}(\gamma),
$$

for $\gamma$ a $m$-cell of $\mathcal{C}_{(n+1)}(f, g)$. The weighted face conditions are then trivial. 
For horizontal composition, suppose we are given a pair of composable arrows $\vec{f}: \vec{x} \rightarrow \vec{y}$ and $\vec{h}: \vec{y} \rightarrow \vec{z}$ in $\int A$. Then we obtain

$$
A_{h}\left(\mathfrak{d}_{\vec{x}, \vec{y}}^{1}(f)\right) \stackrel{A_{h}\left(f_{-}\right)}{\longrightarrow} A_{h}\left(y_{-}\right)=\mathfrak{d}_{\vec{y}, \vec{z}}^{1}(h) \stackrel{h_{-}}{\longrightarrow} z_{-}
$$

in $A_{z}$. Moreover,

$$
A_{h}\left(\mathfrak{d}_{\vec{x}, \vec{y}}^{1} f\right)=A_{h} A_{f}\left(x_{-}\right)=\mathfrak{d}_{\vec{x}, \vec{z}}^{1}(h \circ f),
$$

and therefore we define

$$
\left(\vec{h} *_{0} \vec{f}\right)_{-}:=h_{-} *_{0} A_{h}\left(f_{-}\right) .
$$

This is the familiar definition of composition in the (1-dimensional) category of elements. Now, suppose that we are given $m$-cells $\vec{\varphi}$ and $\vec{\psi}$ of $\int A$, for $m>1$, which are bounded by 0 - and 1-cells as indicated in the following diagram:

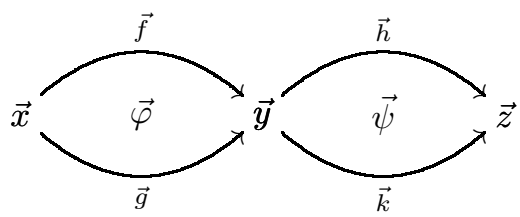

Then,

$$
t^{m} A_{h}\left(\varphi_{-}\right)=A_{h}\left(t g_{-}\right)=A_{h}\left(y_{-}\right)=\mathfrak{d}_{\vec{y}, \vec{z}}^{1}(h),
$$

and we set

$$
\left(\vec{\psi} *_{0} \vec{\varphi}\right)_{-}:=\psi_{-} *_{0} A_{h}\left(\varphi_{-}\right)
$$

For general composition, assume $n>0$ and suppose we are given $m$-cells which are composable along an $n$-cell as indicated in the following diagram:

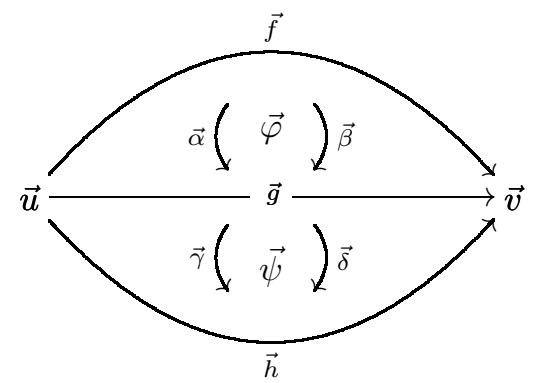

where $\vec{f}, \vec{g}$ and $\vec{h}$ are $n$-cells in $\int A$. Here, $\vec{\alpha}$ and $\vec{\beta}$ are the $(n+1)$-cells bounding $\vec{\varphi}$. I.e., $\vec{\beta}=t^{(m-n-1)} \vec{\varphi}$, etc. As such, when $m=n+1$ we have $\vec{\beta}=\vec{\alpha}=\vec{\varphi}$ and similarly for $\vec{\psi}$. We would like to define the composite $\left(\vec{\psi} *_{n} \vec{\varphi}\right)$. Since the first component will be the composite $\left(\psi *_{n} \varphi\right)$ taken in $\mathcal{C}$, it remains only to define the second component $\left(\vec{\psi} *_{n} \vec{\varphi}\right)_{-}$. The definition will alternate between those cases where $(n+1)$ is even and those where it is odd. First, assume $(n+1)$ is even. Then 
we obtain the following diagram in $A_{y}$ :

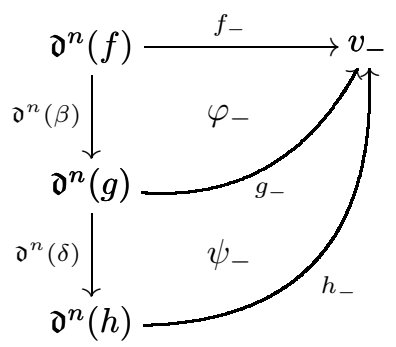

where $\vec{x}$ and $\vec{y}$ are the 0 -cells bounding all of the cells in question. To see that we have correctly identified the $n$-cells of $A_{y}$ bounding $\varphi_{-}$and $\psi_{-}$note that when $m=n+1$ this is trivially the case. When $m>n+1$,

$$
s^{(m-n)}\left(\varphi_{-}\right)=s\left(\alpha_{-}\right)=f_{-} .
$$

Similarly, $t^{(m-n)} \varphi_{-}=t \beta_{-}$which, since $(n+1)$ is even, is equal to $\mathfrak{d}_{\vec{f}, \vec{g}}^{n+1}(\beta)$, as required. Similar calculations show that $\psi_{-}$is as indicated in the diagram. Note also that

$$
\mathfrak{d}_{\vec{u}, \vec{v}}^{n}(\delta) *_{(n-1)} \mathfrak{d}_{\vec{u}, \vec{v}}^{n}(\beta)=\mathfrak{d}^{n}\left(\delta *_{n} \beta\right),
$$

by functoriality of $\mathfrak{d}_{\vec{u}, \vec{v}}^{n}(-)$. These observations suggest that, when $(n+1)$ is even, we define $\left(\vec{\psi} *_{n} \vec{\varphi}\right)_{-}$to be the composite

$$
\left(\psi_{-} *_{(n-1)} \mathfrak{d}_{\vec{u}, \vec{v}}^{n}(\beta)\right) *_{n} \varphi_{-} .
$$

Similarly, when $(n+1)$ is odd, we obtain

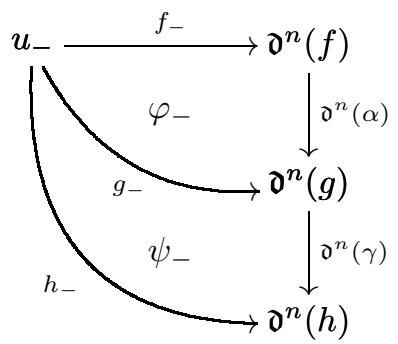

in $A_{y}$ and we may define $\left(\vec{\psi} *_{n} \vec{\varphi}\right)$ - to be the analogous composite. Explicitly, given $\vec{\varphi}$ and $\vec{\psi}$ as above, we define

$$
\left(\vec{\psi} *_{n} \vec{\varphi}\right)_{-}:= \begin{cases}\left(\psi_{-} *_{(n-1)} \mathfrak{d}_{\vec{u}, \vec{v}}^{n}(\beta)\right) *_{n} \varphi_{-} & \text {if }(n+1) \text { is even, } \\ \psi_{-} *_{n}\left(\mathfrak{d}_{\vec{u}, \vec{v}}^{n}(\gamma) *_{(n-1)} \varphi_{-}\right) & \text {if }(n+1) \text { is odd }\end{cases}
$$

where $\vec{\beta}$ and $\vec{\gamma}$ are the bounding $(n+1)$-cells as indicated in (3.2)).

With these definitions we obtain the following proposition, the detailed proof of which can be found in [27]:

Proposition 3.1. Given a (small) $\omega$-category $\mathcal{C}$ and a functor $A: \mathcal{C} \rightarrow \omega$-Cat, the Grothendieck construction $\int A$ of $A$ is a (small) $\omega$-category. 


\section{4. $\omega$-groupoids}

The purpose of this section is to introduce the particular notion of $\omega$-groupoid, which we will be using in order to interpret type theory, and to present several results and constructions on such $\omega$-groupoids. The definition adopted here is the following:

Definition 4.1. A strict $\omega$-category $\mathcal{C}$ is a (strict) $\omega$-groupoid if every $(n+1)$ cell $f: a \rightarrow b$ possesses a strict $*_{n}$-inverse $f^{-1}: b \rightarrow a$. I.e.,

$$
\left(f^{-1} *_{n} f\right)=a,
$$

and

$$
\left(f *_{n} f^{-1}\right)=b .
$$

This definition generalizes both the usual definition of (1-)groupoid as well as the definition of (strict) 2-groupoid occurring in the work of Moerdijk and Svensson [18]. It should be contrasted with the weaker notions of $\omega$-groupoid, also defined in the general setting of strict $\omega$-categories, due to Street [21], and Kapranov and Voevodsky [11]. The essential difference with the definition from [21] is that there the notion of inverse is weakened so that, instead of equations, it is required that there exist (systems of) higher-dimensional cells $\left(f^{-1} *_{n} f\right) \Rightarrow a$, etc. In [11] it is further required that the higher-dimensional cells witnessing invertibility of $f$ satisfy additional coherence conditions.

With Definition 4.1 at hand we obtain the following corollary to Proposition 3.1:

Corollary 4.2. If $\mathcal{C}$ is a (small) $\omega$-groupoid and $A: \mathcal{C} \rightarrow \omega$-Gpd, then $\int A$ is a (small) $\omega$-groupoid.

In particular, the inverse $(\vec{f})^{-1}$ of $\vec{f}: \vec{x} \rightarrow \vec{y}$ is the pair $\left(f^{-1}, A_{f^{-1}}\left(f_{-}\right)^{-1}\right)$ and, for $n>0$, the inverse of a $(n+1)$-cell $\vec{\varphi}: \vec{\alpha} \Rightarrow \vec{\beta}$ is given by

$$
(\vec{\varphi})^{-1}:=\left(\varphi^{-1}, \varphi_{-}^{-1} *_{(n-1)} \mathfrak{d}_{s \vec{\alpha}, t \vec{\alpha}}^{n}\left(\varphi^{-1}\right)\right)
$$

when $(n+1)$ is even, and

$$
(\vec{\varphi})^{-1}:=\left(\varphi^{-1}, \mathfrak{d}_{s \vec{\alpha}, t \vec{\alpha}}^{n}\left(\varphi^{-1}\right) *(n-1) \varphi_{-}^{-1}\right)
$$

when $(n+1)$ is odd.

As mentioned above, when describing the Grothendieck construction $\int A$ we could well have chosen to orient our cells (in terms of the weighted faces) differently. For example, we could define an arrow $\vec{x} \rightarrow \vec{y}$ in $\int A$ to consist of $f: x \rightarrow y$ and $f_{-}: y_{-} \rightarrow x_{-} . f$. By choosing this orientation instead we obtain an $\omega$-category which we call the dual Grothendieck construction of $A$ and denote by $\int^{*} A$. We will give a quick sketch of this construction. When $A: \mathcal{C} \rightarrow \omega-\mathbf{G p d}$ and $\mathcal{C}$ is an $\omega$-groupoid, there is a functor $\neg: \int A \rightarrow \int^{*} A$ which will be required for the interpretation of type theory. This functor is obtained from the isomorphism $\sigma: \mathcal{C} \rightarrow \mathcal{C}^{\text {op }}$ which extends the usual isomorphism which is the identity on objects and sends an arrow $f: a \rightarrow b$ in $\mathcal{C}$ to $f^{-1}: a \rightarrow b$ in $\mathcal{C}^{\text {op }}$ to the setting of $\omega$-groupoids. Informally, $\neg$ simply turns the various triangles from the Grothendieck construction "inside out." 
4.1. The dual Grothendieck construction. When a category $\mathcal{C}$ is an ordinary 1-dimensional groupoid, then there exists an isomorphism $\sigma: \mathcal{C} \rightarrow \mathcal{C}^{\text {op }}$ which is the identity on objects and sends an arrow $f: x \rightarrow y$ to its inverse $f^{-1}: y \rightarrow x$. Now, when $\mathcal{C}$ is an $\omega$-groupoid there is a corresponding functor $\sigma$ which we now consider. We write $\mathcal{C}^{\text {op }}$ for the $\omega$-category obtained by reversing only the 1 -cells of $\mathcal{C}$. For example, given a 2-cell $\alpha: f \rightarrow g$ in $\mathcal{C}, \sigma \alpha: f^{-1} \rightarrow g^{-1}$ is defined to be $\left(g^{-1} *_{0} \alpha^{-1} *_{0} f^{-1}\right)$. As a diagram:

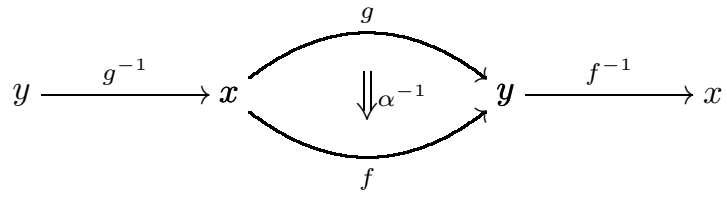

Then, where $\varphi: \alpha \rightarrow \beta$ is a 3 -cell,

$$
\sigma(\varphi):=g^{-1} *_{0}\left(\beta^{-1} *_{1} \varphi^{-1} *_{1} \alpha^{-1}\right) *_{0} f^{-1} .
$$

At higher-dimensions the construction is the same, adding a new "inner" block obtained by composing $\varphi^{-1}$ with the inverses of its boundary maps. The dual Grothendieck construction $\int^{*} A$ of a functor $A: \mathcal{C} \rightarrow \omega$-Cat can be described as the coend

$$
\int^{*} A=\int^{x}(\mathcal{C} \downarrow \sigma(x)) \times A_{x},
$$

for $\sigma: \mathcal{C}^{\text {op }} \rightarrow \mathcal{C}$ as above. In concrete terms, $\int^{*} A$ has the same 0-cells as $\int A$, but 1-cells $\vec{f}: \vec{x} \rightarrow \vec{y}$ are given by $f: x \rightarrow y$ in $\mathcal{C}$ and $f_{-}: y_{-} \rightarrow A_{f}\left(x_{-}\right)$in $A_{x}$. As with $\int A$, the dual weighted face of such an arrow $f_{-}$is $A_{f}\left(x_{-}\right)$and the dual weighted face functor

$$
\mathcal{C}_{1}(x, y) \stackrel{\check{\mathfrak{d}}_{\vec{x}, \vec{y}}^{1}}{\longrightarrow} A_{y}
$$

is obtained by defining $\check{\mathfrak{d}}_{\vec{x}, \vec{y}}^{1}(\gamma)$ to be $x_{-} \cdot \gamma$. Inductively, we then have the following:

$(\boldsymbol{n}+\mathbf{1})$ is even. A $(n+1)$-cell $\vec{\alpha}: \vec{f} \Rightarrow \vec{g}$, with $\vec{f}, \vec{g}: \vec{v} \rightrightarrows \vec{w}$, is a pair consisting of a $(n+1)$-cell $\alpha: f \rightrightarrows g$ in $\mathcal{C}$ and a $(n+1)$-cell $\alpha_{-}$in $A_{y}$ as indicated in the following diagram:

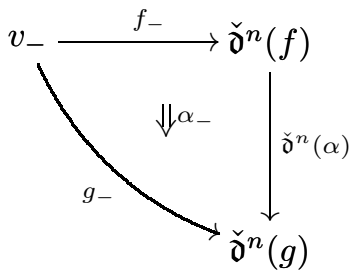

The dual weighted face functor

$$
\mathcal{C}_{n+1}(f, g) \stackrel{\check{\mathfrak{d}}_{\vec{f}, \vec{g}}^{n+1}}{\longrightarrow}\left(A_{y}\right)_{n}\left(v_{-}, \check{\mathfrak{d}}^{n}(g)\right)
$$

is given by defining $\check{\mathfrak{d}}_{\vec{f}, \vec{g}}^{n+1}(\gamma)$ to be $\check{\mathfrak{d}}^{n}(\gamma) *_{(n-1)} f_{-}$.

$(\boldsymbol{n}+\mathbf{1})$ is odd. On the other hand, when $(n+1)$ is odd a $(n+1)$-cell $\vec{\alpha}: \vec{f} \Rightarrow \vec{g}$ is given by $\alpha: f \Rightarrow g$ as above together with a $(n+1)$-cell of $A_{y}$ as indicated in the 
following diagram:

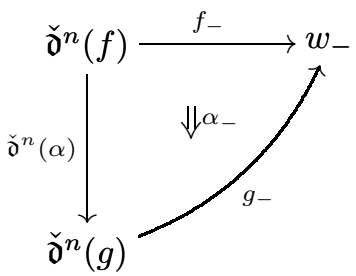

Here the dual weighted face functor

$$
\mathcal{C}_{n+1}(f, g) \stackrel{\mathfrak{d}_{\breve{f}, \vec{g}}^{n+1}}{\longrightarrow}\left(A_{y}\right)_{n}\left(\check{\mathfrak{d}}^{n}(f), w_{-}\right)
$$

is obtained by defining $\check{\mathfrak{d}}_{\vec{f}, \vec{g}}^{n+1}(\gamma)$ to be $g_{-} *_{(n-1)} \check{\mathfrak{d}}^{n}(\gamma)$.

In the case where $\mathcal{C}$ is an $\omega$-groupoid there are functors $\bar{\sigma}_{x}:(x \downarrow \mathcal{C}) \rightarrow\left(\mathcal{C}^{\text {op }} \downarrow x\right)$, for each object $x$ of $\mathcal{C}$, corresponding to $\sigma$ which send an object $x \rightarrow y$ of $(x \downarrow \mathcal{C})$ to its inverse and which act on 1-cells by sending

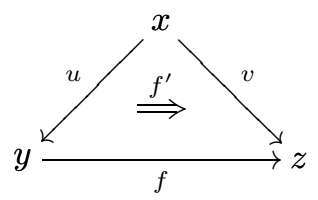

to

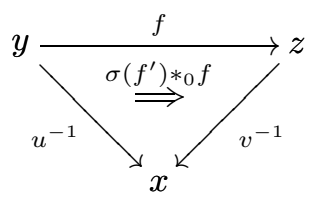

For higher-dimensional cells matters are even more straightforward since a $n$-cell $\left(\alpha, \alpha^{\prime}\right)$, with $n>1$, is sent to $\left(\alpha, \sigma\left(\alpha^{\prime}\right) *_{0} \alpha\right)$. The maps $\bar{\sigma}_{x}$ induce, by the universal property of $\int A$, a canonical functor $\neg: \int A \rightarrow \int^{*} A$ such that the squares

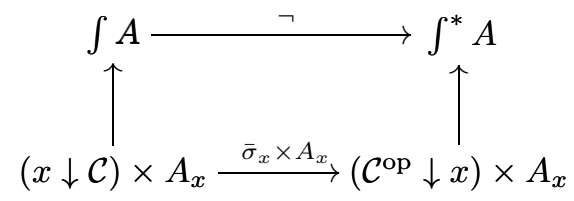

commute, where the vertical maps are the coend inclusions. Consequently, $\neg$ commutes with projections in the sense that the triangle

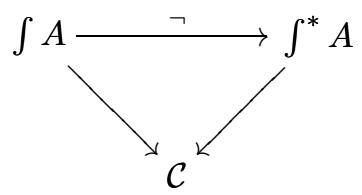

commutes. (This can also be seen from the direct, combinatorial construction of $\neg$ given in [27].) We refer to $\neg$ as the duality functor and we will often denote the second component of $\neg(\vec{\varphi})$ by $\neg \varphi_{-}$. Because the first component of $\neg(\vec{\varphi})$ is $\varphi$ in all dimensions this should lead to no confusion. 


\section{The interpretation of identity types}

We will now describe the interpretation of type theory using $\omega$-groupoids. Roughly, the interpretation, which generalizes directly the Hofmann - Streicher [9] interpretation using regular 1-dimensional groupoids, is obtained by interpreting dependent types as "indexed $\omega$-categories." However, before going into the details several remarks are in order.

First, whereas in ibid. the entire logical framework is interpreted, we here only interpret the basic form of intensional type theory theory (called $\mathbb{T}_{\omega}$ in $[1,27]$ ). We note, however, that we could just as well have interpreted the entire logical framework in this setting. Secondly, the interpretation we give below can be organized into a (large) comprehension category, or a category with attributes, or a category with families. In this case we believe that the model most naturally can be described as a category with attributes or a category with families [4]. We assume that the reader is familiar with these forms of semantics (all of which are essentially inspired by Lawvere's notion of hyperdoctrine [13]). Because the ideas behind the basic interpretation are not new, we do not go into the full details of the interpretation of the basic syntax. Regarding dependent products and sums we note that, because terms are interpreted (here and in the original groupoid model) as sections of projections $\int A \rightarrow \mathcal{C}$ and not as morphisms of split fibrations they correspond, via an equivalence of categories, to 1-cells in the category $[\mathcal{C}, \omega-\mathbf{G p d}]_{\mathrm{ps}}$ which has the same objects as $[\mathcal{C}, \omega$-Gpd] but which has as 1 -cells $(\omega$-categorical) pseudo natural transformations. Explicitly, the notion of pseudo natural transformation employed here can be extracted from the fact that $[\mathcal{C}, \omega-\mathbf{G p d}]_{\mathrm{ps}}$ is equivalent to be the category of algebras and pseudo morphisms of algebras for the 2 -monad on $\omega$-Gpd $/ \mathcal{C}$ which was mentioned in Section 3. We omit the direct combinatorial description of pseudo natural transformations as it will not be required here and merely mention these facts as they serve to provide some motivation for the constructions given below. As such, where $\pi: \int A \rightarrow \mathcal{C}$ is a "dependent projection," dependent sums give the left-adjoint to the reindexing functor

$$
[\mathcal{C}, \omega-\mathbf{G p d}]_{\mathrm{ps}} \stackrel{\pi^{*}}{\longrightarrow}\left[\int A, \omega-\mathbf{G} \mathbf{p d}\right]_{\mathrm{ps}}
$$

and dependent products give the right-adjoint. For the right-adjoint we emphasize that it is crucial that we are dealing with $\omega$-groupoids since the dual functors from Section 4 are necessary (essentially this is because dependent products are homs and therefore exhibit a certain contravariance). Both of these adjoints are described explicitly in Section 7.1 below. The verification that these give the required adjoints is by a lengthy, but routine, combinatorial argument which we omit. Finally, we mention that the type of natural numbers is to be interpreted as the discrete $\omega$ groupoid of natural numbers as in the original groupoid model.

5.1. Contexts, types and terms. The idea of the interpretation, which should be familiar in light of the discussions in the foregoing chapters, is to regard closed types as $\omega$-groupoids. Explicitly, contexts $\Gamma$ are interpreted as small $\omega$ groupoids. To begin with, the empty context () is interpreted as the terminal $\omega$-groupoid:

$$
\llbracket() \rrbracket:=1 .
$$


Now, given a context $\Gamma$ together with its interpretation $\llbracket \Gamma \rrbracket$ as an $\omega$-groupoid, judgements of the form $\Gamma \vdash A$ : type are interpreted as functors

$$
\llbracket \Gamma \rrbracket \stackrel{\llbracket \Gamma \vdash A: \text { type } \rrbracket}{\longrightarrow} \omega \text {-Gpd. }
$$

The extended context $(\Gamma, x: A)$ is then interpreted as the $\omega$-groupoid given by applying the Grothendieck construction from Section 3 to the functor in question:

$$
\llbracket \Gamma, x: A \rrbracket:=\int \llbracket \Gamma \vdash A: \text { type } \rrbracket .
$$

A judgement of the form $\Gamma \vdash a: A$ is then interpreted as a section

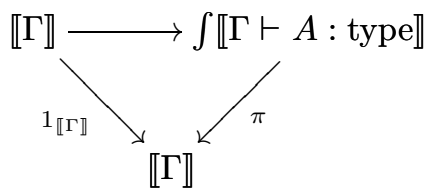

of the projection functor.

5.2. Sections of projection functors. Because terms are interpreted as sections of projection functors it will be useful to give a detailed analysis of such sections. A section $F$ of a projection $\pi: \int A \rightarrow \mathcal{C}$ as indicated in the following diagram:

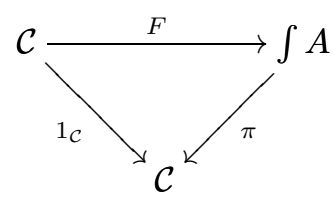

consists exactly of the following data:

Objects. To each object $x$ of $\mathcal{C}$ there is assigned an object $x_{F}$ of $A_{x}$. I.e., $\left(x, x_{F}\right)=F(x)$.

1-Cells. To an arrow $f: x \rightarrow y$ in $\mathcal{C}$ there is assigned an arrow $f_{F}: \mathfrak{d}_{F(x), F(y)}^{1}(f)$ $\rightarrow y_{F}$ of $A_{y}$.

$(\boldsymbol{n}+\mathbf{1})$-Cells. When $(n+1)$ is even, there is assigned to an $(n+1)$-cell $\alpha: f \Rightarrow g$ an $(n+1)$-cell $\alpha_{F}: f_{F} \Rightarrow \mathfrak{d}_{F(f), F(g)}^{n+1}(\alpha)$ of $A_{y}$. When $(n+1)$ is odd, $\alpha_{F}: \mathfrak{d}_{F(f), F(g)}^{n+1}(\alpha) \Rightarrow g_{F}$.

Note that such an assignment is made into a map of globular sets by defining $F(\varphi):=\left(\varphi, \varphi_{F}\right)$. These assignments are required to be functorial in the sense of preserving identities and composition. Preservation of composition amounts to the following. Given $m$-cells, for $m>0, \psi$ and $\varphi$ in $\mathcal{C}$ such that $\left(\psi *_{0} \varphi\right)$ is defined, it is required in order for the assignment $(-)_{F}$ to constitute a section that

$$
\left(\psi *_{0} \varphi\right)_{F}=\psi_{F} *_{0} A_{h}\left(\varphi_{F}\right) .
$$

Assume that the composite $\left(\psi *_{n} \varphi\right)$ is defined and that $t^{m-n-1} \varphi=\beta$ and $s^{m-n-1} \psi$ $=\gamma$. Furthermore, let $u$ and $v$ be the $(n-1)$-cells bounding both $\varphi$ and $\psi$. Then it is required that

$$
\left(\psi *_{n} \varphi\right)_{F}= \begin{cases}\left(\psi_{F} *_{(n-1)} \mathfrak{d}_{F u, F v}^{n}(\beta)\right) *_{n} \varphi_{F} & \text { if }(n+1) \text { is even } \\ \psi_{F} *_{n}\left(\mathfrak{d}_{F u, F v}^{n}(\gamma) *_{(n-1)} \varphi_{F}\right) & \text { if }(n+1) \text { is odd }\end{cases}
$$


We also note that given any functor $\sigma: \mathcal{D} \rightarrow \mathcal{C}$, there exists a functor $\{\sigma\}_{A}: \int(A \circ$ $\sigma) \rightarrow \int A$ such that the following diagram is a pullback in $\omega$-Cat:

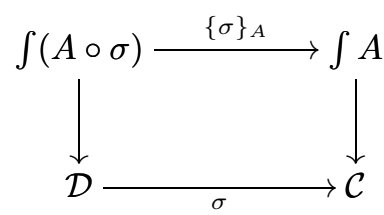

Namely, $\{\sigma\}_{A}$ sends $\vec{x}$ in $\int A \sigma$ to $(\sigma(x), a)$ and similarly for cells in all dimensions. Consequently, there corresponds to any section $F$ of the projection $\int A \rightarrow \mathcal{C}$ a canonical section $F[\sigma]$ of $\int A \sigma \rightarrow \mathcal{D}$ for which

$$
F \circ \sigma=\{\sigma\}_{A} \circ F[\sigma] \text {. }
$$

Finally, note that, by taking $\mathcal{D}$ to be $\int A$ itself and $\sigma$ to be $\pi$, we obtain $\int A \pi$ as the pullback of $\pi$ along itself and there exists a canonical map $\delta_{A}: \int A \rightarrow \int A \pi$ induced by the identity functor $1 \int A$.

5.3. Substitution and weakening. Suppose we are given an $\omega$-groupoid $\mathcal{C}$ interpreting a context $\Gamma$ together with $A: \mathcal{C} \rightarrow \omega$-Gpd, $B: \int A \rightarrow \omega$-Gpd and $C: \int B \rightarrow \omega$-Gpd interpreting judgements

$$
\Gamma \vdash A \text { : type, } \quad \Gamma, x: A \vdash B(x): \text { type, } \quad \text { and } \quad \Gamma, x: A, y: B(x) \vdash C(x, y): \text { type, }
$$

respectively. Moreover, let a section $a$ interpreting the judgement $\Gamma \vdash a: A$ be given. Then the judgement $\Gamma \vdash B[a / x]$ is interpreted as the composite functor

$$
\mathcal{C} \stackrel{a}{\longrightarrow} \int A \stackrel{B}{\longrightarrow} \omega-\mathbf{G p d} .
$$

Similarly,

$$
\llbracket \Gamma, y: B(a) \vdash C(a, y): \text { type } \rrbracket:=C \circ\{a\}_{B},
$$

in the notation of Section 5.2. Finally, if $c$ is a section of $\int C \rightarrow \int B$ interpreting the judgement $\Gamma, x: A, y: B(x) \vdash c(x, y): C(x, y)$ we define

$$
\llbracket \Gamma, y: B(a) \vdash c(a, y): C(a, y) \rrbracket:=c[a] .
$$

Finally, for weakening, we note that when functors $A, B: \mathcal{C} \rightarrow \boldsymbol{\omega}$-Gpd interpret the judgements $\Gamma \vdash A$ : type and $\Gamma \vdash B$ : type, the "weakened" judgement $\Gamma, x: A \vdash$ $B$ : type is interpreted by the composite

$$
\int A \stackrel{\pi}{\longrightarrow} \mathcal{C} \stackrel{B}{\longrightarrow} \omega \text {-Gpd. }
$$

5.4. Identity types. When $A: \mathcal{C} \rightarrow \omega$-Gpd has as its domain an $\omega$-groupoid $\mathcal{C}$, the identity type $\left(\right.$ for $A$ ) is a functor $I_{A}: \int A \pi \rightarrow \omega$-Gpd where $\pi: \int A \rightarrow \mathcal{C}$ is the projection. By definition, $\int A \circ \pi$ is has as objects tuples $\vec{x}=\left(x, x_{-}, x_{+}\right)$where $x_{-}$and $x_{+}$are themselves objects of $A_{x}$. Similarly, $n$-cells $\vec{f}$ in $\int A \pi$ are tuples $\left(f, f_{-}, f_{+}\right)$such that both $\left(f, f_{-}\right)$and $\left(f, f_{+}\right)$are $n$-cells in $\int A$. I.e., the following diagram is a pullback

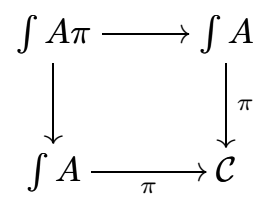


with the nameless functors the obvious projections. With this in mind, it is straightforward to describe the action of $I_{A}$ on objects. Namely, $I_{A}\left(x, x_{-}, x_{+}\right)$is defined to be the $\omega$-groupoid $A_{x}\left(x_{-}, x_{+}\right)$. Perhaps though, in light of the discussion of the combinatorics of the Grothendieck construction from the previous sections, matters are more complicated in higher dimensions. It is to this task which we now turn.

Remark 5.1. Because, when $\int A \pi$ is involved, we are dealing with two instances of the Grothendieck construction $\int A$ it will be convenient to introduce some notation to describe the various weighted face functors. In particular, because we adopt the convention of notating cells $\vec{f}$ of $\int A \pi$ by $\left(f, f_{-}, f_{+}\right)$we will also notate the corresponding weighted face functors accordingly. I.e., we write $\mathfrak{d}_{-}^{n}(f)$ and $\mathfrak{d}_{+}^{n}(f)$ for the instances of these functors corresponding to the appropriate "negative" and "positive" projections $\int A \pi \rightarrow \int A$. When subscripts are necessary we write, e.g., $\mathfrak{d}_{\vec{\alpha} \vec{\beta} ; \xi}^{n}$ with $\xi=+,-$. We adopt also a corresponding convention for the "co-weighted face" functors.

5.5. Identity types in dimensions 1 and 2. Given an arrow $\vec{f}: \vec{x} \rightarrow \vec{y}$ in $\int A \pi, I_{A}(\vec{f})$ is the functor

$$
A_{x}\left(x_{-}, x_{+}\right) \rightarrow A_{y}\left(x_{-}, x_{+}\right)
$$

which sends any cell $\gamma$ of $A_{x}\left(x_{-}, x_{+}\right)$to the following composite:

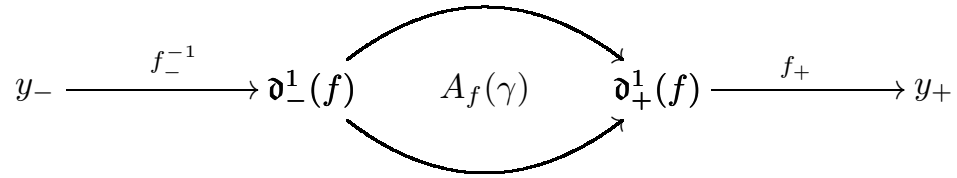

I.e., $I_{A}(\vec{f})(\gamma)$ is defined to be $\left(f_{+} *_{0} A_{f}(\gamma) *_{0} f_{-}^{-1}\right)$. Already at this stage we have tacitly made use of the dual functor $\neg$ since $\neg f_{-}$is $f_{-}^{-1}$.

Now, given a 2-cell $\vec{\alpha}: \vec{f} \Rightarrow \vec{g}$ we must provide a natural transformation $I_{A}(\vec{\alpha})$ as indicated in the following diagram:

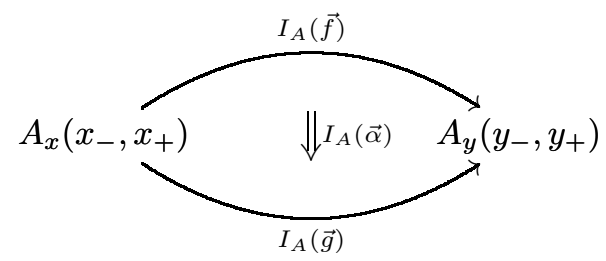

Fixing an object $h: x_{-} \rightarrow x_{+}$of $A_{x}\left(x_{-}, x_{+}\right)$, the component $I_{A}(\vec{\alpha})_{h}$ of this transformation at $h$ is described by the composite of the following diagram in $A_{y}$ :

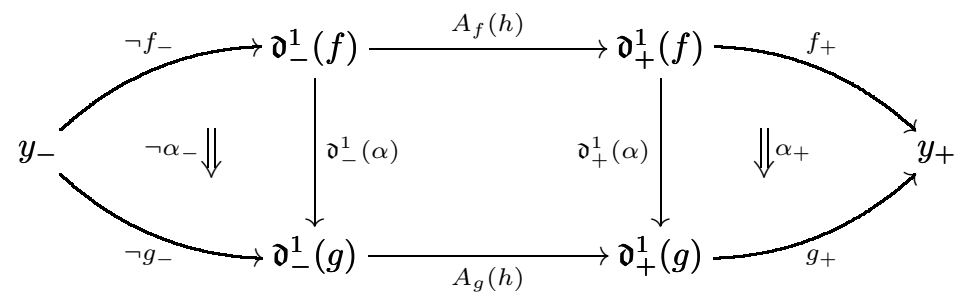

where the middle square commutes (on the nose) by naturality of $A_{\alpha}$. Explicitly,

$$
I_{A}(\vec{\alpha})_{h}:=\left(f_{+} *_{0} A_{g}(h) *_{0} \neg \alpha_{-}\right) *_{1}\left(\alpha_{+} *_{0} A_{f}(h) *_{0} \neg f_{-}\right) .
$$


With this definition in mind, we now turn to the introduction of some auxiliary functors which will allow us to describe the identity types in higher dimensions.

5.6. Auxiliary functors. Holding an arrow $\vec{f}: \vec{x} \rightarrow \vec{y}$ of $\int A \pi$ fixed together with an object $h$ of $A_{x}\left(x_{-}, x_{+}\right)$we define functors

$$
\begin{aligned}
& \left(A_{y}\right)_{1}\left(\mathfrak{d}_{+}^{1}(f), y_{+}\right) \stackrel{\Psi_{\vec{f}, h}^{1}}{\longrightarrow}\left(A_{y}\right)_{1}\left(y_{-}, y_{+}\right), \\
& \left(A_{y}\right)_{1}\left(y_{-}, \mathfrak{d}_{-}^{1}(f)\right) \stackrel{\check{\Psi}_{\vec{f}, h}^{1}}{\longrightarrow}\left(A_{y}\right)_{1}\left(y_{-}, y_{+}\right)
\end{aligned}
$$

by setting

$$
\begin{gathered}
\Psi_{\vec{f}, h}^{1}(-):=\left(-*_{0} A_{f} h *_{0} \neg f_{-}\right), \\
\check{\Psi}_{\vec{f}, h}^{1}(-):=\left(f_{+} *_{0} A_{f} h *_{0}-\right) .
\end{gathered}
$$

As usual, we omit either one or both of the subscripts when no confusion will result. The first thing we observe about these functors is that

$$
\Psi_{\vec{f}}^{1}\left(f_{+}\right)=\check{\Psi}_{\vec{f}}^{1}\left(\neg f_{-}\right) .
$$

The next feature which should be emphasized is that these functors interact in an important way with the usual weighted face functors. In particular, the following diagram (of $\omega$-categories) commutes:

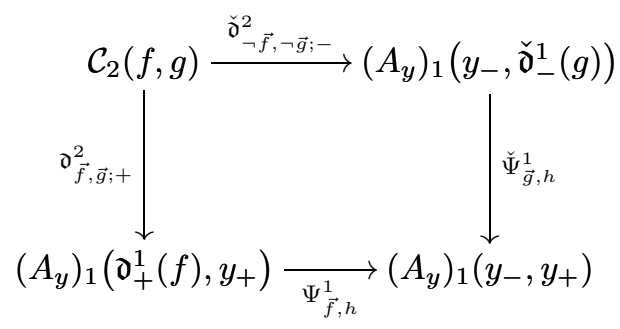

To see this, we note that

$$
\begin{aligned}
\check{\Psi}_{\vec{g}}^{1}\left(\check{\mathfrak{d}}_{-}^{2}(\gamma)\right) & =g_{+} *_{0} A_{g} h *_{0} \mathfrak{d}_{-}^{1}(\gamma) *_{0} \neg f_{-} \\
& =g_{+} *_{0} \mathfrak{d}_{+}^{1}(\gamma) *_{0} A_{f} h *_{0} \neg f_{-} \\
& =\Psi_{\vec{f}}^{1}\left(\mathfrak{d}_{+}^{2}(\gamma)\right),
\end{aligned}
$$

where the second equation is by naturality of $A_{\gamma}$. We now observe that, when $\vec{\alpha}$ is as above, the component $I_{A}(\vec{\alpha})$ at $h$ can be described using these functors as follows:

$$
I_{A}(\vec{\alpha})_{h}=\check{\Psi}_{\vec{g}, h}^{1}\left(\neg \alpha_{-}\right) *_{1} \Psi_{\vec{f}, h}^{1}\left(\alpha_{+}\right)
$$

In particular, $I_{A}(\vec{\alpha})_{h}$ is obtained by composing

$$
\Psi_{\vec{f}}^{1}\left(f_{+}\right) \stackrel{\Psi_{\vec{f}}^{1}\left(\alpha_{+}\right)}{\longrightarrow} \Psi_{\vec{f}}^{1}\left(\mathfrak{d}_{+}^{2}(\alpha)\right)=\check{\Psi}_{\vec{g}}^{1}\left(\check{\mathfrak{d}}_{-}^{2}(\alpha)\right) \stackrel{\check{\Psi}_{\vec{g}}^{1}\left(\neg \alpha_{-}\right)}{\longrightarrow} \check{\Psi}_{\vec{g}}^{1}\left(\neg g_{-}\right)=\Psi_{\vec{g}}^{1}\left(g_{+}\right) .
$$

As such, we have employed both (5.2) and (5.3) in order to show that the composite defining $I_{A}(\vec{\alpha})_{h}$ makes sense. We emphasize this point because it provides the first look at what will be required in higher dimensions. 
At the next stage, holding a 2-cell $\vec{\alpha}: \vec{f} \rightarrow \vec{g}$ and an arrow $h: x_{-} \rightarrow x_{+}$as above fixed, we define functors

$$
\begin{array}{r}
\left(A_{y}\right)_{2}\left(f_{+}, \mathfrak{d}_{\vec{f}, \vec{g} ;+}^{2}(\alpha)\right) \stackrel{\Psi_{\vec{\alpha}, h}^{2}}{\longrightarrow}\left(A_{y}\right)_{2}\left(\Psi_{\vec{f}, h}^{1}\left(f_{+}\right), \Psi_{\vec{g}, h}^{1}\left(g_{+}\right)\right), \\
\left(A_{y}\right)_{2}\left(\check{\mathfrak{d}}_{\neg \vec{f}, \neg \vec{g} ;-}^{2}(\alpha), \neg g_{-}\right) \stackrel{\check{\Psi}_{\vec{\alpha}, h}^{2}}{\longrightarrow}\left(A_{y}\right)_{2}\left(\Psi_{\vec{f}, h}^{1}\left(f_{+}\right), \Psi_{\vec{g}, h}^{1}\left(g_{+}\right)\right)
\end{array}
$$

as follows

$$
\begin{gathered}
\Psi_{\vec{\alpha}, h}^{2}(-):=\check{\Psi}_{\vec{g}, h}^{1}\left(\neg \alpha_{-}\right) *_{1} \Psi_{\vec{f}, h}^{1}(-), \\
\check{\Psi}_{\vec{\alpha}, h}^{2}(-):=\check{\Psi}_{\vec{g}, h}^{1}(-) *_{1} \Psi_{\vec{f}, h}^{1}\left(\alpha_{+}\right) .
\end{gathered}
$$

The motivation for these definitions can perhaps best be seen in consultation with (5.1). It follows, using the same reasoning from (5.4), that these functors are welldefined and possess the appropriate boundaries. An immediate consequence of the definition is that

$$
\Psi_{\vec{\alpha}, h}^{2}\left(\alpha_{+}\right)=\check{\Psi}_{\vec{\alpha}, h}^{2}\left(\neg \alpha_{-}\right) .
$$

Moreover, (5.3) also generalizes to dimension 2 to give:

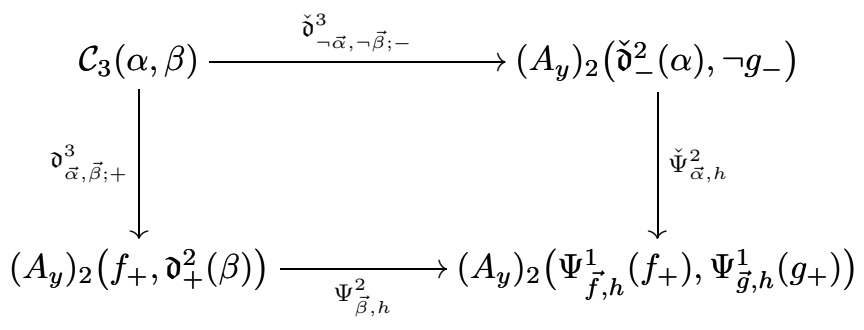

when $\vec{\alpha}, \vec{\beta}: \vec{f} \rightrightarrows \vec{g}$ are fixed 2-cells. To see that the equation holds we reason as follows:

$$
\begin{aligned}
\Psi_{\vec{\beta}, h}^{2}\left(\mathfrak{d}_{+}^{3}(\gamma)\right) & =\check{\Psi}_{\vec{g}, h}^{1}\left(\neg \beta_{-}\right) *_{1} \Psi_{\vec{f}, h}^{1}\left(\mathfrak{d}_{+}^{3}(\gamma)\right) \\
& =\check{\Psi}_{\vec{g}, h}^{1}\left(\neg \beta_{-}\right) *_{1} \Psi_{\vec{f}, h}^{1}\left(\mathfrak{d}_{+}^{2}(\gamma)\right) *_{1} \Psi_{\vec{f}, h}^{1}\left(\alpha_{+}\right) \\
& =\check{\Psi}_{\vec{g}, h}^{1}\left(\neg \beta_{-}\right) *_{1} \check{\Psi}_{\vec{g}, h}^{1}\left(\check{\mathfrak{d}}_{-}^{2}(\gamma)\right) *_{1} \Psi_{\vec{f}, h}^{1}\left(\alpha_{+}\right) \\
& =\check{\Psi}_{\vec{g}, h}^{1}\left(\neg \beta_{-} *_{1} \check{\mathfrak{d}}_{-}^{2}(\gamma)\right) *_{1} \Psi_{\vec{f}, h}^{1}\left(\alpha_{+}\right) \\
& =\check{\Psi}_{\vec{g}, h}^{1}\left(\check{\mathfrak{d}}_{-}^{3}(\gamma)\right) *_{1} \Psi_{\vec{f}, h}^{1}\left(\alpha_{+}\right) \\
& =\check{\Psi}_{\vec{\alpha}, h}^{2}\left(\check{\mathfrak{d}}_{-}^{3}(\gamma)\right),
\end{aligned}
$$

where the third equation is by (5.3). We will now show that this construction can be generalized to all dimensions $(n+1)$ with $n \geq 2$. In particular we will prove that at each stage $(n+1)$, for every $(n+1)$-cell $\vec{\varphi}: \vec{\alpha} \rightarrow \vec{\beta}$ and arrow $h: x_{-} \rightarrow x_{+}$, there exist functors $\Psi_{\vec{\varphi}, h}^{n+1}$ and $\check{\Psi}_{\vec{\varphi}, h}^{n+1}$ satisfying the following conditions:

(1) When $(n+1)$ is odd,

$$
\begin{array}{r}
\left(A_{y}\right)_{n+1}\left(\mathfrak{d}_{\vec{\alpha}, \vec{\beta} ;+}^{n+1}(\varphi), \beta_{+}\right) \stackrel{\Psi_{\vec{\varphi}, h}^{n+1}}{\longrightarrow}\left(A_{y}\right)_{n+1}\left(\Psi_{\vec{\alpha}, h}^{n}\left(\alpha_{+}\right), \Psi_{\vec{\beta}, h}^{n}\left(\beta_{+}\right)\right), \\
\left(A_{y}\right)_{n+1}\left(\neg \alpha_{-}, \check{\mathfrak{d}}_{\neg \vec{\alpha}, \neg \vec{\beta} ;-}^{n+1}(\varphi)\right) \stackrel{\check{\Psi}_{\vec{\varphi}, h}^{n+1}}{\longrightarrow}\left(A_{y}\right)_{n+1}\left(\Psi_{\vec{\alpha}, h}^{n}\left(\alpha_{+}\right), \Psi_{\vec{\beta}, h}^{n}\left(\beta_{+}\right)\right) .
\end{array}
$$


Similarly, when $(n+1)$ is even,

$$
\begin{array}{r}
\left(A_{y}\right)_{n+1}\left(\alpha_{+}, \mathfrak{d}_{\vec{\alpha}, \vec{\beta} ;+}^{n+1}(\varphi)\right) \stackrel{\Psi_{\vec{\varphi}, h}^{n+1}}{\longrightarrow}\left(A_{y}\right)_{n+1}\left(\Psi_{\vec{\alpha}, h}^{n}\left(\alpha_{+}\right), \Psi_{\vec{\beta}, h}^{n}\left(\beta_{+}\right)\right), \\
\left(A_{y}\right)_{n+1}\left(\check{\mathfrak{d}}_{\neg \vec{\alpha}, \neg \vec{\beta} ;-}^{n+1}(\varphi), \neg \beta_{-}\right) \stackrel{\check{\Psi}_{\vec{\varphi}, h}^{n+1}}{\longrightarrow}\left(A_{y}\right)_{n+1}\left(\Psi_{\vec{\alpha}, h}^{n}\left(\alpha_{+}\right), \Psi_{\vec{\beta}, h}^{n}\left(\beta_{+}\right)\right)
\end{array}
$$

(2) When $\vec{\varphi}$ is an $(n+1)$-cell as above,

$$
\Psi_{\vec{\varphi}, h}^{n+1}\left(\varphi_{+}\right)=\check{\Psi}_{\vec{\varphi}, h}^{n+1}\left(\neg \varphi_{-}\right) .
$$

(3) Let parallel $(n+1)$-cells $\vec{\varphi}, \vec{\psi}: \vec{\alpha} \rightrightarrows \vec{\beta}$ be given. When $(n+1)$ is odd, the following diagram commutes:

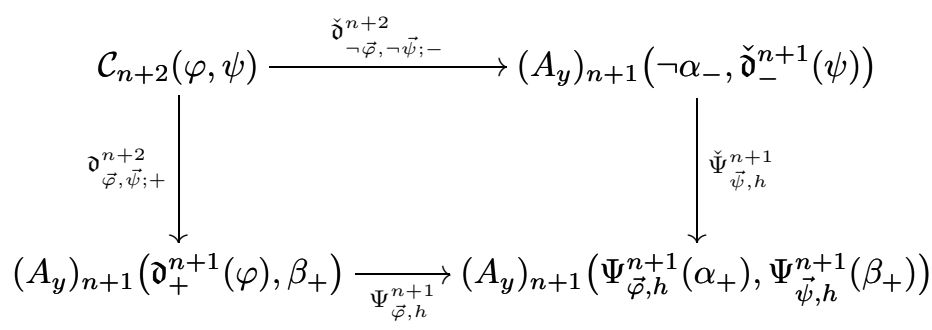

And, when $(n+1)$ is even,

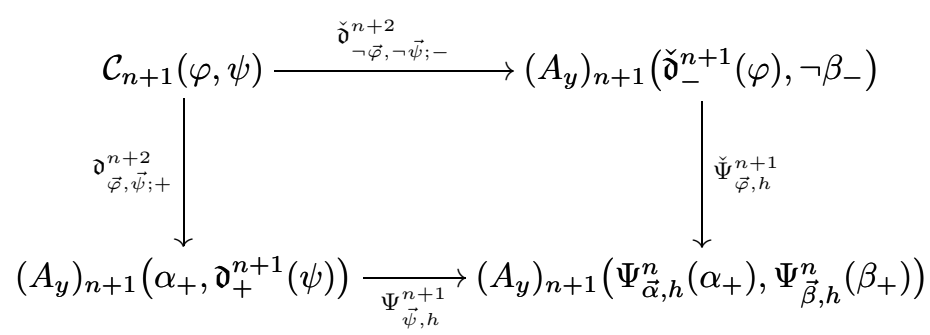

commutes.

Lemma 5.2. The conditions (1)-(3) described above are satisfied when, for $\vec{\varphi}: \vec{\alpha} \rightarrow \vec{\beta}$ an $(n+1)$-cell of $\int A \pi$ and $h: x_{-} \rightarrow x_{+}$as above, the functors $\Psi_{\vec{\varphi}, h}^{n+1}$ and $\check{\Psi}_{\vec{\varphi}, h}^{n+1}$ are defined as follows:

$$
\Psi_{\vec{\varphi}, h}^{n+1}(-):= \begin{cases}\check{\Psi}_{\vec{\beta}, h}^{n}\left(\neg \varphi_{-}\right) *_{n} \Psi_{\vec{\alpha}, h}^{n}(-) & \text { if }(n+1) \text { is even } \\ \Psi_{\vec{\beta}, h}^{n}(-) *_{n} \check{\Psi}_{\vec{\alpha}, h}^{n}\left(\neg \varphi_{-}\right) & \text {if }(n+1) \text { is odd }\end{cases}
$$

and

$$
\check{\Psi}_{\vec{\varphi}, h}^{n+1}(-):= \begin{cases}\check{\Psi}_{\vec{\beta}, h}^{n}(-) *_{n} \Psi_{\vec{\alpha}, h}^{n}\left(\varphi_{+}\right) & \text {if }(n+1) \text { is even } \\ \Psi_{\vec{\beta}, h}^{n}\left(\varphi_{+}\right) *_{n} \check{\Psi}_{\vec{\alpha}, h}^{n}(-) & \text { if }(n+1) \text { is odd } .\end{cases}
$$

Proof. We give the proof in the case where $(n+1)$ is odd as the case where it is even is essentially dual. First, to see that $\Psi_{\vec{\varphi}}^{n+1}$ is well defined and possesses the source and target as stated in condition (1) above, suppose we are given a $m$ cell $\zeta$ of $\left(A_{y}\right)_{n+1}\left(\mathfrak{d}_{+}^{n+1} \varphi, \beta_{+}\right)$. Then, $\zeta$ is a $(m+1)$-cell of $\left(A_{y}\right)_{n}\left(f_{+}, \mathfrak{d}_{+}^{n} \beta\right)$ where 
$\vec{\alpha}, \vec{\beta}: \vec{f} \rightrightarrows \vec{g}$. As such, we may apply $\Psi_{\vec{\beta}}^{n}$ to obtain

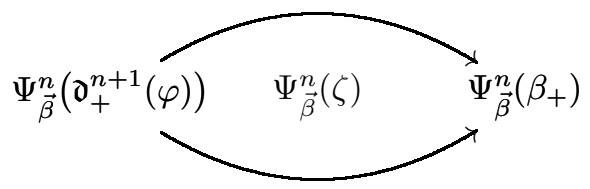

By definition of $\neg \vec{\varphi}$, we have also $\neg \varphi_{-}: \neg \alpha_{-} \rightarrow \check{\mathfrak{d}}_{-}^{n+1}(\varphi)$. By the induction hypothesis,

$$
\check{\Psi}_{\vec{\alpha}}^{n}\left(\check{\mathfrak{d}}_{-}^{n+1}(\varphi)\right)=\Psi_{\vec{\beta}}^{n}\left(\mathfrak{d}_{+}^{n+1}(\varphi)\right),
$$

and therefore applying $\check{\Psi}_{\vec{\alpha}}^{n}$ to $\neg \varphi$ - yields

$$
\Psi_{\vec{\alpha}}^{n}\left(\alpha_{+}\right)=\check{\Psi}_{\vec{\alpha}}^{n}\left(\neg \alpha_{-}\right) \stackrel{\check{\Psi}_{\vec{\alpha}}^{n}\left(\neg \varphi_{-}\right)}{\longrightarrow} \Psi_{\vec{\beta}}^{n}\left(\mathfrak{d}_{+}^{n+1}(\varphi)\right) .
$$

Here the equation is by the induction hypothesis. As such, the composite

$$
\Psi_{\vec{\varphi}}^{n+1}(\zeta):=\Psi_{\vec{\beta}}^{n}(\zeta) *_{n} \check{\Psi}_{\vec{\alpha}}^{n}\left(\neg \varphi_{-}\right)
$$

is defined and possesses the correct boundary. A similar argument shows that $\check{\Psi}_{\vec{\varphi}}^{n+1}$ is well-defined with the appropriate boundary. Note also that, with these definitions, condition (2) from above is trivially satisfied.

Finally, to see that (3) is satisfied we note that, when $\vec{\varphi}$ and $\vec{\psi}$ are parallel $(n+1)$-cells as above and $\gamma$ is a cell of $\mathcal{C}_{n+2}(\varphi, \psi)$,

$$
\begin{aligned}
\Psi_{\vec{\varphi}}^{n+1}\left(\mathfrak{d}_{+}^{n+2}(\gamma)\right) & =\Psi_{\vec{\beta}}^{n}\left(\mathfrak{d}_{+}^{n+2}(\gamma)\right) *_{n} \check{\Psi}_{\vec{\alpha}}^{n}\left(\neg \varphi_{-}\right) \\
& =\Psi_{\vec{\beta}}^{n}\left(\psi_{+} *_{n} \mathfrak{d}_{+}^{n+1}(\gamma)\right) *_{n} \check{\Psi}_{\vec{\alpha}}^{n}\left(\neg \varphi_{-}\right) \\
& =\Psi_{\vec{\beta}}^{n}\left(\psi_{+}\right) *_{n} \Psi_{\breve{\beta}^{n}}^{n}\left(\check{\mathfrak{d}}_{+}^{n+1}(\gamma)\right) *_{n} \check{\Psi}_{\vec{\alpha}}^{n}\left(\neg \varphi_{-}\right) \\
& =\Psi_{\vec{\beta}}^{n}\left(\psi_{+}\right) *_{n} \check{\Psi}_{\vec{\alpha}}^{n}\left(\check{\mathfrak{d}}_{-}^{n+1}(\gamma)\right) *_{n} \check{\Psi}_{\vec{\alpha}}^{n}\left(\neg \varphi_{-}\right) \\
& =\Psi_{\vec{\beta}}^{n}\left(\psi_{+}\right) *_{n} \check{\Psi}_{\vec{\alpha}}^{n}\left(\check{\mathfrak{d}}_{-}^{n+2}(\gamma)\right) \\
& =\check{\Psi}_{\vec{\psi}}^{n+1}\left(\check{\mathfrak{d}}_{-}^{n+2}(\gamma)\right),
\end{aligned}
$$

where the fourth equation is by the induction hypothesis.

5.7. Definition of the identity types. With the functors $\Psi^{n}$ and $\check{\Psi}^{n}$ at our disposal it is possible to give a very efficient description of the "identity type" functor $I_{A}: \int A \pi \rightarrow \omega$-Gpd. In particular, the official definition of $I_{A}$ in all dimensions is as follows:

Objects. $I_{A}\left(x, x_{-}, x_{+}\right)$is given by the $\omega$-groupoid $A_{x}\left(x_{-}, x_{+}\right)$.

1-Cells. Given $\vec{f}: \vec{x} \rightarrow \vec{y}$, the functor $I_{A}(\vec{f}): I(\vec{x}) \rightarrow I(\vec{y})$ is defined by setting

$$
I_{A}(\vec{f})(\gamma):=f_{+} *_{0} A_{f}(\gamma) *_{0} \neg f_{-},
$$

for any $m$-cell $\gamma$ of $A_{x}\left(x_{-}, x_{+}\right)$.

2-Cells. A 2-cell $\vec{\alpha}: \vec{f} \Rightarrow \vec{g}$ is sent to the natural transformation $I_{A}(\vec{\alpha})$ which is defined, for an object $h: x_{-} \rightarrow x_{+}$of $A_{x}\left(x_{-}, x_{+}\right)$, as follows:

$$
I_{A}(\vec{\alpha})_{h}:=\Psi_{\vec{\alpha}, h}^{2}\left(\alpha_{+}\right)=\check{\Psi}_{\vec{\alpha}, h}^{2}\left(\neg \alpha_{-}\right) .
$$

That $I_{A}(\vec{\alpha})_{h}$ possesses the correct domain and codomain is an immediate consequence of the results of Section 5.6. 
$(\boldsymbol{n}+\mathbf{1})$-Cells. In general, given an $(n+1)$-cell $\vec{\varphi}: \vec{\alpha} \Rightarrow \vec{\beta}$ in $\int A \pi$ and $h: x_{-} \rightarrow$ $x_{+}$, we define

$$
I_{A}(\vec{\varphi})_{h}:=\Psi_{\vec{\varphi}, h}^{(n+1)}\left(\varphi_{+}\right)=\check{\Psi}_{\vec{\varphi}, h}^{(n+1)}\left(\neg \varphi_{-}\right) .
$$

Again, that $I_{A}(\vec{\varphi})_{h}$ possesses the correct domain and codomain follows directly from the definition of $I_{A}$ at lower dimensional cells together with the results of Section 5.6.

It remains only to verify that $I_{A}$ is functorial. To this end, we first prove that the data given in the definition are of the appropriate kinds. E.g., that $I(\vec{\alpha})$ is a natural transformation, etc.

Lemma 5.3. As defined above, when $\vec{\alpha}: \vec{f} \Rightarrow \vec{g}$ is a 2-cell of $\int A \pi, I_{A}(\vec{\alpha})$ is an $\omega$-natural transformation.

Proof. Explicitly, we must show that, for any $m$-cell $\gamma$ of $A_{x}\left(x_{-}, x_{+}\right)$with $m>0$ such that $s^{m}(\gamma)=h$ and $t^{m}(\gamma)=k$, the following "schematic" diagram commutes:

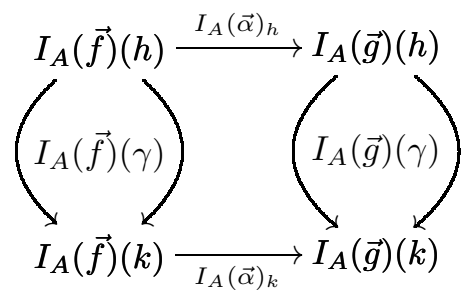

in $A_{y}\left(y_{-}, y_{+}\right)$. I.e., we must prove that

$$
I_{A}(\vec{\alpha})_{k} *_{1} I_{A}(\vec{f})(\gamma)=I_{A}(\vec{g})(\gamma) *_{1} I_{A}(\vec{\alpha})_{h} .
$$

By definition of $\Psi_{\vec{\alpha}, h}^{2}\left(\alpha_{+}\right)$and interchange it follows that the right-hand side of (5.8) is equal to

$$
\left(g_{+} *_{0} A_{g}(\gamma) *_{0} \neg \alpha_{-}\right) *_{1} \Psi_{\vec{f}, h}^{1}\left(\alpha_{+}\right) .
$$

Because $A_{\alpha}$ is itself a transformation $A_{f} \Rightarrow A_{g}$ we obtain

$$
\mathfrak{d}_{+}^{1}(\alpha) *_{0} A_{f}(\gamma)=A_{g}(\gamma) *_{0} \mathfrak{d}_{-}^{1}(\alpha) .
$$

Thus,

$$
\begin{aligned}
A_{g}(\gamma) *_{0} \neg \alpha_{-} & =\left(A_{g}(k) *_{1} A_{g}(\gamma)\right) *_{0}\left(\neg \alpha_{-} *_{1} \check{\mathfrak{d}}_{-}^{2}(\alpha)\right) \\
& =\left(A_{g}(k) *_{0} \neg \alpha_{-}\right) *_{1}\left(A_{g}(\gamma) *_{0} \check{\mathfrak{d}}_{-}^{2}(\alpha)\right) \\
& =\left(A_{g}(k) *_{0} \neg \alpha_{-}\right) *_{1}\left(\mathfrak{d}_{+}^{1}(\alpha) *_{0} A_{f}(\gamma) *_{0} f_{-}^{-1}\right) .
\end{aligned}
$$

Thus, the right-hand side of (5.8) is equal to

$$
\check{\Psi}_{\vec{g}, k}^{1}\left(\neg \alpha_{-}\right) *_{1}\left(\mathfrak{d}_{+}^{2}(\alpha) *_{0} A_{f}(\gamma) *_{0} f_{-}^{-1}\right) *_{1} \Psi_{\vec{f}, h}^{1}\left(\alpha_{+}\right) .
$$

Moreover, the interchange and unit laws yield

$$
\left(\mathfrak{d}_{+}^{2}(\alpha) *_{0} A_{f}(\gamma) *_{0} f_{-}^{-1}\right) *_{1} \Psi_{\vec{\alpha}, h}^{1}\left(\alpha_{+}\right)=\alpha_{+} *_{0} A_{f}(\gamma) *_{0} f_{-}^{-1} .
$$

Thus, the right-hand side of (5.8) is equal to

$$
\check{\Psi}_{\vec{\alpha}, k}^{1}\left(\neg \alpha_{-}\right) *_{1}\left(\alpha_{+} *_{0} A_{f}(\gamma) *_{0} f_{-}^{-1}\right)=I_{A}(\vec{\alpha})_{k} *_{1} I_{A}(\vec{f})(\gamma),
$$

as required.

A similar argument yields the following fact: 
Lemma 5.4. Given parallel $n$-cells $\vec{\alpha}$ and $\vec{\beta}$ in $\int A \pi$ bounded by 1 -cells $\vec{f}, \vec{g}: \vec{x}$ $\rightrightarrows \vec{y}$ together with a $(n+1)$-cell $\vec{\varphi}: \vec{\alpha} \Rightarrow \vec{\beta}, I_{A}(\vec{\varphi})$, as defined above, is a modification $I_{A}(\vec{\alpha}) \Rightarrow I_{A}(\vec{\beta})$.

Proposition 5.5. As defined, $I_{A}$ is a functor $\int(A \circ \pi) \rightarrow \omega-\mathbf{G p d}$.

Proof. First we consider the case of vertical composition. Let $p$-cells $\vec{\varphi}$ and $\vec{\psi}$ be given, for $p \geq m \geq n+1>1$, which are bounded by 0 -cells $\vec{x}$ and $\vec{y}$ and by $n$-cells $\vec{f}, \vec{g}$ and $\vec{h}$ as indicated in the following diagram:

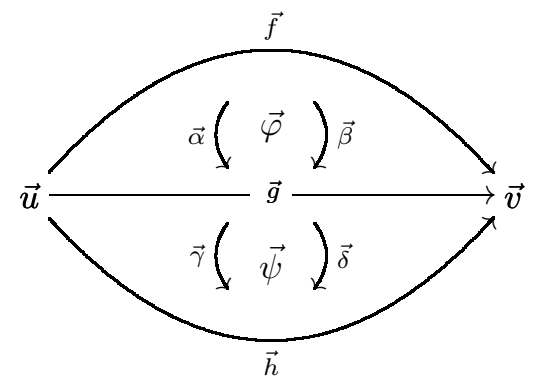

Then, for any object $k: x_{-} \rightarrow x_{+}$of $A_{x}\left(x_{-}, x_{+}\right)$, we will prove by induction on $m$ the stronger fact that when $m$ is odd

$$
\begin{aligned}
\Psi_{s^{p-m}\left(\vec{\psi} *_{n} \vec{\varphi}\right), k}^{m}\left(\left(\vec{\psi} *_{n} \vec{\varphi}\right)_{+}\right) & =\Psi_{s^{(p-m)} \vec{\psi}, k}^{m}\left(\psi_{+}\right) *_{n} \Psi_{s^{(p-m)} \vec{\varphi}, k}^{m}\left(\varphi_{+}\right), \\
\check{\Psi}_{t^{p-m}\left(\vec{\psi} *_{n} \vec{\varphi}\right), k}^{m}\left(\neg\left(\vec{\psi} *_{n} \vec{\varphi}\right)_{-}\right) & =\check{\Psi}_{t^{(p-m)} \vec{\psi}, k}^{m}\left(\neg \psi_{-}\right) *_{n} \check{\Psi}_{t^{(p-m)} \vec{\varphi}, k}^{m}\left(\neg \varphi_{-}\right) ;
\end{aligned}
$$

and

$$
\begin{aligned}
& \Psi_{t^{p-m}\left(\vec{\psi} *_{n} \vec{\varphi}\right), k}^{m}\left(\left(\vec{\psi} *_{n} \vec{\varphi}\right)_{+}\right)=\Psi_{t^{(p-m)} \vec{\psi}, k}^{m}\left(\psi_{+}\right) *_{n} \Psi_{t^{(p-m)} \vec{\varphi}, k}^{m}\left(\varphi_{+}\right),
\end{aligned}
$$

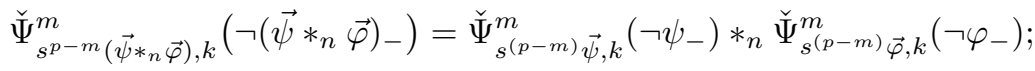

when $m$ is even.

First, assume $m=n+1$ is even. We also assume that $n+1>2$ since the case where $n+1=2$ is a straightforward calculation (using ideas essentially the same as those used here). Then

$$
\Psi_{\vec{\delta} *_{n} \vec{\beta}}^{n+1}\left(\left(\vec{\psi} *_{n} \vec{\varphi}\right)_{+}\right)=\check{\Psi}_{\vec{h}}^{n}\left(\neg\left(\vec{\delta} *_{n} \vec{\beta}\right)_{-}\right) *_{n} \Psi_{\vec{f}}^{n}\left(\left(\vec{\psi} *_{n} \vec{\varphi}\right)_{+}\right)
$$

And this is equal, by definition of composition, to

$$
\check{\Psi}_{\vec{h}}^{n}\left(\neg \delta_{-} *_{n}\left(\check{\mathfrak{d}}_{-}^{n}(\delta) *_{(n-1)} \neg \beta_{-}\right)\right) *_{n} \Psi_{\vec{f}}^{n}\left(\left(\psi_{+} *_{(n-1)} \mathfrak{d}_{+}^{n}(\beta)\right) *_{n} \varphi_{+}\right)
$$

Now we will investigate in more detail each of the larger terms in this composite.

First:

$$
\left.\check{\Psi}_{\vec{h}}^{n}\left(\neg \delta_{-} *_{n}\left(\check{\mathfrak{d}}_{-}^{n}(\delta) *_{(n-1)} \neg \beta_{-}\right)\right)=\check{\Psi}_{\vec{h}}^{n}\left(\neg \delta_{-}\right) *_{n} \check{\Psi}_{\vec{h}}^{n}\left(\check{\mathfrak{d}}_{-}^{n}(\delta) *_{(n-1)} \neg \beta_{-}\right)\right) .
$$

By definition of $\check{\Psi}^{n}$ and functoriality this is equal to

$$
\check{\Psi}_{\vec{h}}^{n}\left(\neg \delta_{-}\right) *_{n}\left(\Psi_{\vec{v}}^{n-1}\left(h_{+}\right) *_{(n-1)} \check{\Psi}_{\vec{u}}^{n-1}\left(\check{\mathfrak{d}}_{-}^{n}(\delta)\right) *_{(n-1)} \check{\Psi}_{\vec{u}}^{n-1}\left(\neg \beta_{-}\right)\right),
$$

which by (5.7) is equal to:

$$
\begin{aligned}
\check{\Psi}_{\vec{h}}^{n}\left(\neg \delta_{-}\right) *_{n}\left(\Psi_{\vec{v}}^{n-1}\left(h_{+}\right) *_{(n-1)}\right. & \left.\Psi_{\vec{v}}^{n-1}\left(\mathfrak{d}_{+}^{n}(\delta)\right) *_{(n-1)} \check{\Psi}_{\vec{u}}^{n-1}\left(\neg \beta_{-}\right)\right) \\
= & \check{\Psi}_{\vec{h}}^{n}\left(\neg \delta_{-}\right) *_{n}\left(\Psi_{\vec{v}}^{n-1}\left(\mathfrak{d}_{+}^{n+1}(\delta)\right) *_{(n-1)} \check{\Psi}_{\vec{u}}^{n-1}\left(\neg \beta_{-}\right)\right)
\end{aligned}
$$


Similarly, the other half of (5.10) is equal to

$$
\left(\Psi_{\vec{v}}^{n-1}\left(\psi_{+}\right) *_{(n-1)} \check{\Psi}_{\vec{u}}^{n-1}\left(\check{\mathfrak{d}}_{-}^{n+1}(\beta)\right)\right) *_{n} \Psi_{\vec{f}}^{n}\left(\varphi_{+}\right) .
$$

By these observations and a routine calculation it follows that (5.10) is equal to

$$
\check{\Psi}_{\vec{h}}^{n}\left(\neg \delta_{-}\right) *_{n}\left(\Psi_{\vec{v}}^{n-1}\left(\psi_{+}\right) *(n-1) \check{\Psi}_{\vec{u}}^{n-1}\left(\neg \beta_{-}\right)\right) *_{n} \Psi_{\vec{f}}^{n}\left(\varphi_{+}\right) .
$$

Finally, using the unit and interchange laws this is seen to be the same as $\Psi_{\vec{\delta}}^{n+1}\left(\psi_{+}\right)$ $*_{n} \Psi_{\vec{\beta}}^{n+1}\left(\varphi_{+}\right)$. The base cases where $n+1$ is odd are dual and the induction steps are trivial. Thus, $I_{A}$ is a functor.

\section{Reflexivity and elimination terms}

In this section we define the functors which will interpret reflexivity and elimination terms. As in [9] we will interpret terms as sections of the projection map $\int A \rightarrow \mathcal{C}$ associated to the functor $A$ which interprets their type. We begin by summarizing some of the basic facts about such sections and the related structures resulting from the Grothendieck construction.

6.1. Reflexivity terms. We end this section by describing briefly the "reflexivity term" associated to a functor $A: \mathcal{C} \rightarrow \omega$-Gpd. By definition, the reflexivity term should be a section $r_{A}$ :

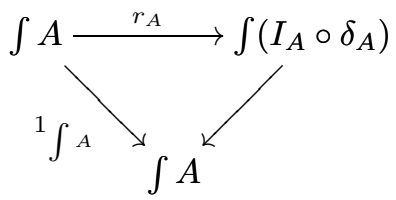

where $\delta_{A}$ is as in Section 5.2. However, we will require the explicit description of this map and we will also introduce some notation related to this map. For objects, given an object $\vec{x}$ of $\int A$, we must provide an object $\vec{x}_{r}$ of $I_{A}\left(x, x_{-}, x_{-}\right)$. I.e., $\vec{x}_{r}$ should be an arrow $x_{-} \rightarrow x_{-}$in $A_{x}$. We define $\vec{x}_{r}$ to be the identity $x_{-}$. (Note that here and throughout we omit the identity maps and write $x_{-}$instead of $i\left(x_{-}\right)$.) Next, given an arrow $\vec{f}: \vec{x} \rightarrow \vec{y}$, we need to provide an arrow

$$
(\partial I)_{r(\vec{x}), r(\vec{y})}^{1}(\vec{f}) \stackrel{\vec{f}_{r}}{\longrightarrow} \vec{y}_{r}=y_{-}
$$

in $A_{y}$, where $(\partial I)^{n}$ denotes the weighted face functor for $\int I_{A}$ (and so, in this case, also $\left.\int I_{A} \delta_{A}\right)$. But, by definition, $(\partial I)^{1}(\vec{f})$ is just $y_{-}$and we therefore define $\vec{f}_{r}$ to be $y_{-}$. Indeed, at every dimension $n \geq 1$, when $\vec{\varphi}$ is a $n$-cell of $\int A$ bounded by objects $\vec{x}$ and $\vec{y}$, we define $\vec{\varphi}_{r}$ to be $y_{-}$. Alternatively, the map $r_{A}$ may be constructed, as the anonymous referee was kind enough to mention to us, as the canonical section induced by the fact that

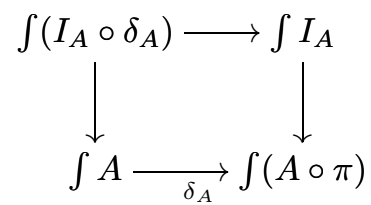

is a pullback. Note that $r$ is constant $y_{-}$in all dimensions $n \geq 1$ since $(\partial I)_{r(\vec{x}), r(\vec{y})}^{1}(\vec{\varphi})$ is equal to $y_{-}$for any $\vec{\varphi}$ in $\int A$ bounded by $\vec{x}$ and $\vec{y}$. As such, we define $\vec{\varphi}_{r}$ to be 
$y_{-}$in all dimensions $n \geq 1$. With this definition functoriality of $r$ is trivial and we have proved:

Lemma 6.1. Given $A: \mathcal{C} \rightarrow \omega$-Gpd as above, the assignment $r$ defined above induces a section $r_{A}$ as indicated in (6.1).

6.2. Setting up the construction of elimination terms. Turning now to elimination terms, suppose we are given a functor $D: \int I_{A} \rightarrow \omega$-Gpd together with a section $d: \int A \rightarrow \int D\left\{\delta_{A}\right\}_{I_{A}} r_{A}$ of the projection $\int D\left\{\delta_{A}\right\}_{I_{A}} r_{A} \rightarrow \int A$. We would like to prove that this extends to a section $J$ of $\int D \rightarrow \int I_{A}$. We begin by fixing notation.

As we are dealing with multiple cases of the Grothendieck construction it will be convenient to introduce some notation to deal with the different weighted face functions which occur. First, we denote by $\Theta^{n}(-)$ the weighted face functor for $\int D$. As usual, we denote by $\mathfrak{d}_{-}^{n}$ and $\mathfrak{d}_{+}^{n}$ the functors for the two projections of $\int I_{A}$. Finally, we denote by $\widetilde{\Theta}^{n}$ the weighted face functor for $\int D \circ\left\{\delta_{A}\right\}_{I_{A}} \circ r_{A}$.

Next, we observe that there is an endofunctor $\downarrow(-): \int I_{A} \rightarrow \int I_{A}$ defined as the following composite:

$$
\int I_{A} \stackrel{\pi_{0}}{\longrightarrow} \int A \stackrel{r_{A}}{\longrightarrow} \int I_{A} \circ \delta_{A} \rightarrow \int I_{A}
$$

I.e., $\downarrow$ sends an object $\vec{x}=\left(x, x_{-}, x_{+}, x_{\rightarrow}\right)$ to $\left(x, x_{-}, x_{-}, x_{-}\right)$and similarly for higher-dimensional cells. Here, as throughout, we omit mention of identity arrows. I.e., writing out identities we have that $\downarrow \vec{x}$ is $\left(x, x_{-}, x_{-}, i\left(x_{-}\right)\right)$or $\left(x, x_{-}, x_{-}, 1_{x_{-}}\right)$.

We will often be concerned with the situation where we consider, given $\vec{x}$ an object of $\int I_{A}$, the restriction $\left(x, x_{-}\right)$of $\vec{x}$ to $\int A$. Rather than write $\pi_{0}(\vec{x})$ every time for this object we instead denote this pair by $\breve{x}$. Similarly, $\breve{\gamma}$ denotes $\pi_{0}(\vec{\gamma})$ for general $n$-cells $\vec{\gamma}$ of $\int I_{A}$.

6.3. Naturality cells. The construction of the elimination terms is rather technical and proceeds in several stages. First, we describe "naturality cells" which exhibit what amounts to a (suitable notion of) pseudo natural transformation $\varepsilon_{-}$ from $\downarrow(-)$ to the identity $1 \int_{I_{A}}$. The construction proceeds by induction on dimension as usual.

Dimension 0. First, in dimension 0 , given $\vec{x}$ in $\int I_{A}$, we define an arrow $\varepsilon_{\vec{x}}: \downarrow(\vec{x}) \rightarrow \vec{x}$ as follows:

$$
\varepsilon_{\vec{x}}:=\left(x, x_{-}, x_{\rightarrow}, x_{\rightarrow}\right),
$$

where $\vec{x}=\left(x, x_{-}, x_{+}, x_{\rightarrow}\right)$. Next, holding 0 -cells $\vec{x}$ and $\vec{y}$ of $\int I_{A}$ fixed we define functors

$$
\left(\int I_{A}\right)_{1}(\vec{x}, \vec{y}) \stackrel{\nabla_{\vec{x}, \vec{y} ; \xi}^{1}}{\longrightarrow}\left(\int I_{A}\right)_{1}(\downarrow \vec{x}, \vec{y})
$$

for $\xi=-,+$ as follows:

$$
\nabla_{\vec{x}, \vec{y} ; \xi}^{1}:= \begin{cases}\left(-*_{0} \varepsilon_{\vec{x}}\right) & \text { if } \xi=-, \\ \left(\varepsilon_{\vec{y} *_{0} \downarrow} \downarrow(-)\right) & \text { if } \xi=+.\end{cases}
$$

As usual, we omit the subscripts $\vec{x}$ and $\vec{y}$ when these are understood. 
Note that with these definitions, when $\vec{\varphi}$ is any $m$-cell, with $m \geq 1$,

$$
\begin{aligned}
& \nabla_{-}^{1}(\vec{\varphi})=\left(\varphi, \varphi_{-}, \varphi_{+} *_{0} A_{f}\left(x_{\rightarrow}\right), \varphi_{\rightarrow}\right), \\
& \nabla_{+}^{1}(\vec{\varphi})=\left(\varphi, \varphi_{-}, y_{\rightarrow} *_{0} \varphi_{-}, y_{\rightarrow}\right),
\end{aligned}
$$

where $s^{m-1} \vec{\varphi}=\vec{f}$ and $\vec{f}: \vec{x} \rightarrow \vec{y}$ is as above.

Remark 6.2. Because we will sometimes want to refer to the different elements of such a pair $\nabla_{\xi}^{1}(\vec{\varphi})$ we denote by $\left[\nabla_{\xi}^{1}(\vec{\varphi})\right]_{k}$ the $k$ th component, for $k=0,1,2,3$. E.g., $\left[\nabla_{+}^{1}(\vec{\varphi})\right]_{2}$ is $\left(y_{\rightarrow} *_{0} \varphi_{-}\right)$,

Dimension 1. Next, we define, given an arrow $\vec{f}: \vec{x} \rightarrow \vec{y}$ in $\int I_{A}$, a 2-cell

$$
\nabla_{-}^{1}(\vec{f}) \stackrel{\varepsilon_{\vec{f}}}{\longrightarrow} \nabla_{+}^{1}(\vec{f}) .
$$

I.e., $\varepsilon_{\vec{f}}$ is as indicated in the following "naturality" diagram:

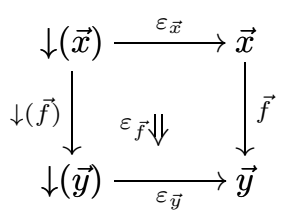

In particular, $\varepsilon_{\vec{f}}$ is defined to be $\left(f, f_{-}, f_{\rightarrow} *_{0} f_{-}, f_{\rightarrow}\right)$. This definition is easily seen to make sense using (6.2) and (6.3). Now, holding parallel arrows $\vec{f}$ and $\vec{g}$ fixed, we define functors

$$
\left(\int I_{A}\right)_{2}(\vec{f}, \vec{g}) \stackrel{\nabla_{\xi}^{2}}{\longrightarrow}\left(\int I_{A}\right)_{2}\left(\nabla_{-}^{1}(\vec{f}), \nabla_{+}^{1}(\vec{g})\right)
$$

by

$$
\nabla_{\xi}^{2}(\vec{\gamma}):= \begin{cases}\nabla_{+}^{1}(\vec{\gamma}) *_{1} \varepsilon_{\vec{f}} & \text { if } \xi=-, \\ \varepsilon_{\vec{g}} *_{1} \nabla_{-}^{1}(\vec{\gamma}) & \text { if } \xi=+.\end{cases}
$$

With these definitions it is straightforward to verify that

$$
\nabla_{-}^{2}(\vec{\varphi})=\left(\varphi, \varphi_{-}, f_{\rightarrow} *_{0} \varphi_{-}, f_{\rightarrow}\right),
$$

when $\vec{\varphi}$ is any cell of $\left(\int I_{A}\right)_{2}(\vec{f}, \vec{g})$. In order to obtain a similar analysis of $\nabla_{+}^{2}(\vec{\varphi})$ we require a further fact about the duality functor $\neg$.

Lemma 6.3. Given any $m$-cell, for $m \geq 1, \vec{\varphi}$ of $\int A$,

$$
\begin{aligned}
& \neg \varphi_{-} *_{0} \varphi_{-}=\mathfrak{d}_{\vec{x}, \vec{y}}^{1}(\varphi), \\
& \varphi_{-} *_{0} \neg \varphi_{-}=y_{-}
\end{aligned}
$$

where $\vec{x}$ and $\vec{y}$ are the 0 -cells bounding $\vec{\varphi}$.

Proof. This is a direct consequence of the easily proved fact that, for $0 \leq n \leq$ $m-2$

$$
\rho_{\partial \vec{\varphi}}^{m-n-2}\left(\varphi_{-}^{-1}\right) *_{n} \varphi_{-}=t^{m-n-1}(\vec{\varphi})_{-}^{-} *_{n}\left(\varphi_{-} *_{(n+1)} \rho_{\partial \vec{\varphi}}^{m-n-3}\left(\varphi_{-}^{-1}\right)\right)
$$

when $n$ is even (or 0 ), and

$$
\varphi_{-} *_{n} \rho_{\partial \vec{\varphi}}^{m-n-2}\left(\varphi_{-}^{-1}\right)=\left(\rho_{\partial \vec{\varphi}}^{m-n-3}\left(\varphi_{-}^{-1}\right) *_{(n+1)} \varphi_{-}\right) *_{n} s^{m-n-1}(\vec{\varphi})_{-}^{-1},
$$

when $n$ is odd. Iteratively applying these facts and canceling inverses yields the required result. 
Using Lemma 6.3 it follows, by a (lengthy but) straightforward calculation, that, where $\vec{\beta}$

$$
\nabla_{+}^{2}(\vec{\varphi})=\left(\varphi, \varphi_{-},\left(g_{\rightarrow} *_{1} \Psi_{\vec{\beta}, x_{\rightarrow}}^{2}\left(\varphi_{+}\right)\right) *_{0} \beta_{-}, \varphi_{\rightarrow}\right)
$$

for any $m$-cell $\vec{\varphi}$ of $\left(\int I_{A}\right)_{2}(\vec{f}, \vec{g})$ with $\vec{\beta}=t^{m-2} \vec{\varphi}$. Using (6.4) and (6.5) we define $\varepsilon_{\vec{\alpha}}$, for $\vec{\alpha}: \vec{f} \rightrightarrows \vec{g}$ a 2 -cell of $\int I_{A}$, as follows:

$$
\varepsilon_{\vec{\alpha}}:=\left(\alpha, \alpha_{-}, \alpha_{\rightarrow} *_{0} \alpha_{-}, \alpha_{\rightarrow}\right) .
$$

In higher dimensions this procedure is carried out as follows:

Dimension $(\boldsymbol{n}+\mathbf{1})$. Given $\vec{\alpha}$ and $\vec{\beta}$ of dimension $n$ together with the appropriate $\nabla_{-}^{n}$ we first observe that, using decompositions of $\nabla_{\xi}^{n}(\vec{\varphi})$ corresponding to (6.4) and (6.5), and proved by a standard calculation using Lemma 6.3, it follows that, if, $\vec{\varphi}: \vec{\alpha} \Rightarrow \vec{\beta}$ is a $(n+1)$-cell, then

$$
\nabla_{-}^{n}(\vec{\varphi})= \begin{cases}\left(\varphi, \varphi_{-}, \alpha_{\rightarrow} *_{0} \varphi_{-}, \alpha_{\rightarrow}\right) & \text { if } n \text { is even, } \\ \left(\varphi, \varphi_{-},(\partial I)^{n}(\vec{\varphi}) *_{0} \varphi_{-}, \varphi_{\rightarrow}\right) & \text { if } n \text { is odd }\end{cases}
$$

and

$$
\nabla_{+}^{n}(\vec{\varphi})= \begin{cases}\left(\varphi, \varphi_{-},(\partial I)^{n}(\vec{\varphi}) *_{0} \varphi_{-}, \alpha_{\rightarrow}\right) & \text { if } n \text { is even } \\ \left(\varphi, \varphi_{-}, \beta_{\rightarrow} *_{0} \varphi_{-}, \beta_{\rightarrow}\right) & \text { if } n \text { is odd }\end{cases}
$$

Thus we define

$$
\nabla_{-}^{n}(\vec{\alpha}) \stackrel{\varepsilon_{\vec{\varphi}}}{\longrightarrow} \nabla_{+}^{n}(\vec{\beta})
$$

by

$$
\left(\varphi, \varphi_{-}, \varphi_{\rightarrow} *_{0} \varphi_{-}, \varphi_{\rightarrow}\right) .
$$

Now, holding $\vec{\alpha}$ and $\vec{\beta}$ fixed, we define

$$
\left(\int I_{A}\right)_{n+1}(\vec{\alpha}, \vec{\beta}) \stackrel{\nabla_{\xi}^{n+1}}{\longrightarrow}\left(\int I_{A}\right)_{n+1}\left(\nabla_{-}^{n}(\vec{\alpha}), \nabla_{+}^{n}(\vec{\beta})\right)
$$

for $\xi=-,+$ as

$$
\nabla_{\xi}^{n+1}(\vec{\gamma}):= \begin{cases}\nabla_{+}^{n}(\vec{\gamma}) *_{n} \varepsilon_{\vec{\alpha}} & \text { if } \xi=-, \\ \varepsilon_{\vec{\beta}} *_{n} \nabla_{-}^{n}(\vec{\gamma}) & \text { if } \xi=+.\end{cases}
$$

6.4. Elimination terms in dimensions 0,1 . Assume we are given an object $\vec{x}=\left(x, x_{-}, x_{+}, x_{\rightarrow}\right)$ of $\int I_{A}$. We would like to provide a corresponding object, which for the sake of notational convenience we simply denote by $x_{J}$, of $D(\vec{x})$. This $x_{J}$ is obtained by a kind of Yoneda style argument. Namely, we observe that, by assumption there is a term $\breve{x}_{d}$ in $D(\downarrow(\vec{x}))$. Applying the functor $D\left(\varepsilon_{\vec{x}}\right)$ yields the required $x_{J}$ in $D(\vec{x})$. I.e.,

$$
x_{J}:=D\left(\varepsilon_{\vec{x}}\right)\left(\breve{x}_{d}\right) .
$$

Because it will greatly simplify matters in the later stages, we introduce a special notation for $D\left(\varepsilon_{\vec{x}}\right)$ and its higher-dimensional generalizations $D\left(\varepsilon_{\vec{\gamma}}\right)$. Namely, we define

$$
\langle\vec{\gamma}\rangle:=D\left(\varepsilon_{\vec{\gamma}}\right) .
$$

With this notation $x_{J}=\langle\vec{x}\rangle\left(\breve{x}_{d}\right)$. 
In dimension 1 , given an arrow $\vec{f}: \vec{x} \rightarrow \vec{y}$ in $\int I_{A}$ we have by hypothesis the arrow $\breve{f}_{d}: \widetilde{\Theta}_{\breve{x}, \breve{y}}^{1}(\breve{f}) \rightarrow \breve{y}_{d}$ in $D(\downarrow \vec{y})$ and, applying $\langle\vec{y}\rangle$,

$$
\langle\vec{y}\rangle\left(\widetilde{\Theta}^{1} \breve{f}\right) \stackrel{\langle\vec{y}\rangle\left(\breve{f}_{d}\right)}{\longrightarrow} y_{J}
$$

in $D(\vec{y})$. Now,

$$
\langle\vec{y}\rangle\left(\widetilde{\Theta}^{1} \breve{f}\right)=\langle\vec{y}\rangle\left(D(\downarrow \vec{f}) \breve{x}_{d}\right)=D\left(\nabla_{+}^{1} \vec{f}\right) \breve{x}_{d}
$$

Also,

$$
\Theta^{1}(\vec{f})=D(\vec{f})\left(x_{J}\right)=D\left(\nabla_{-}^{1} \vec{f}\right) \breve{x}_{d}
$$

and therefore we define $f_{J}$ to be the composite

$$
\Theta^{1}(\vec{f}) \stackrel{\langle\vec{f}\rangle_{\breve{x}_{d}}}{\longrightarrow} D\left(\nabla_{+}^{1} \vec{f}\right) \breve{x}_{d} \stackrel{\langle\vec{y}\rangle \breve{f}_{d}}{\longrightarrow} y_{J} .
$$

Again, it will be useful to introduce some additional notation to clarify the situation in higher-dimensions. First, holding fixed objects $\vec{x}$ and $\vec{y}$ of $\int I_{A}$, we define a functor

$$
\left(\int I_{A}\right)_{1}(\vec{x}, \vec{y}) \stackrel{\vartheta_{\vec{x}, \vec{y}}^{1}}{\longrightarrow} D(\vec{y})
$$

by

$$
\vartheta_{\vec{x}, \vec{y}}^{1}(\vec{\gamma}):=\langle\vec{y}\rangle\left(\widetilde{\Theta}_{\vec{x}, \vec{y}}^{1}(\breve{\gamma})\right) .
$$

The next ingredient is to define, for $\vec{f}: \vec{x} \rightarrow \vec{y}$, arrows $\triangleleft(\vec{f}): \Theta^{1}(\vec{f}) \rightarrow \vartheta^{1}(\vec{f})$ and $\triangleright(\vec{f}): \vartheta^{1}(\vec{f}) \rightarrow y_{J}$ as follows:

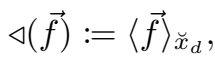

$$
\begin{aligned}
& \triangleright(\vec{f}):=\langle\vec{y}\rangle \breve{f}_{d} .
\end{aligned}
$$

Thus, with this notation $f_{J}$ is just $\triangleright(\vec{f}) *_{0} \triangleleft(\vec{f})$. We will see below that, in general, $\gamma_{J}$ will always be formed as a composite of the form $\triangleright(\vec{\gamma}) *_{k-1} \triangleleft(\vec{\gamma})$ along a $(k-1)$-cell $\vartheta^{k}(\vec{\gamma})$.

6.5. Elimination terms in dimensions 2 . In dimension 2 , let arrows $\vec{f}, \vec{g}: \vec{x}$ $\rightrightarrows \vec{y}$ in $\int I_{A}$ be given together with a 2-cell $\vec{\alpha}: \vec{f} \Rightarrow \vec{g}$. In order to define $\alpha_{J}$ we will describe 2-cells filling both the square and triangle as indicated in the following diagram:

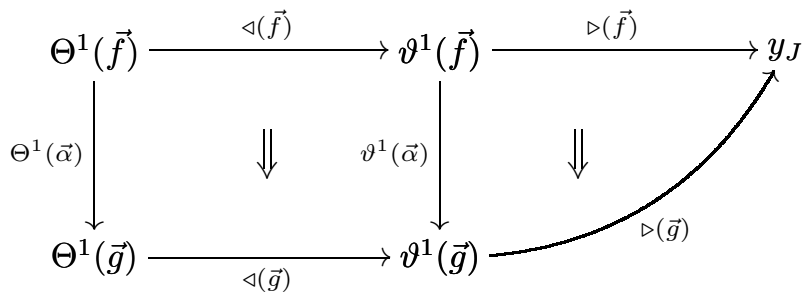

Defining the functor

$$
\left(\int I_{A}\right)_{2}(\vec{f}, \vec{g}) \stackrel{\vartheta_{\vec{f}, \vec{g}}^{2}}{\longrightarrow} D(\vec{y})_{1}\left(\Theta^{1}(\vec{f}), y_{J}\right)
$$

by

$$
\vartheta^{2}(-):=\triangleright(\vec{g}) *_{0} \vartheta^{1}(-) *_{0} \triangleleft(\vec{f})
$$


we see that our goal is precisely to provide 2 -cells

$$
f_{J} \stackrel{\triangleleft(\vec{\alpha})}{\longrightarrow} \vartheta_{\vec{f}, \vec{g}}^{2}(\vec{\alpha}) \stackrel{\triangleright(\vec{\alpha})}{\longrightarrow} \Theta_{\vec{f}, \vec{g}}^{2}(\vec{\alpha}) .
$$

The strategy for filling the square and triangle from above is fairly simple. For the triangle, we use $\breve{\alpha}_{d}$, and for the square we use the naturality cell $\varepsilon_{\vec{\alpha}}$. To begin with, we define

$$
\triangleleft(\vec{\alpha}):=\langle\vec{y}\rangle\left(\breve{\alpha}_{d}\right) *_{0} \triangleleft(\vec{f}) .
$$

For the square, we observe that

$$
\begin{aligned}
\Theta^{1}(\vec{\gamma}) & =D(\vec{\gamma})\left(x_{J}\right), \\
\vartheta^{1}(\vec{\gamma}) & =D\left(\nabla_{+}^{1} \vec{\gamma}\right)\left(\breve{x}_{d}\right)
\end{aligned}
$$

where $\vec{\gamma}$ is any cell of $\int I_{A}(\vec{x}, \vec{y})$. Thus,

$$
\begin{aligned}
\vartheta^{1}(\vec{\gamma}) *_{0} \triangleleft(\vec{f}) & =D\left(\nabla_{-}^{2} \vec{\gamma}\right)\left(\breve{x}_{d}\right), \\
\triangleleft(\vec{g}) *_{0} \Theta^{1}(\vec{\gamma}) & =D\left(\nabla_{+}^{2} \vec{\gamma}\right)\left(\breve{x}_{d}\right) .
\end{aligned}
$$

As such, we define

$$
\triangleright(\vec{\alpha}):=\triangleright(\vec{g}) *_{0}\langle\vec{\alpha}\rangle_{\breve{x}_{d}} .
$$

Thus, $\alpha_{J}$ is $\left(\triangleright(\vec{\alpha}) *_{1} \triangleleft(\vec{\alpha})\right)$. It will often be convenient to omit parentheses when dealing with the arrows $\triangleright(\vec{\alpha})$ and $\triangleleft(\vec{\alpha})$. In order to avoid confusion, we adopt the convention that $\triangleright$ and $\triangleleft$ bind more tightly than composition. I.e., $\triangleleft \vec{\gamma} *_{k} \vec{\varphi}$ should be read as $\triangleleft(\vec{\gamma}) *_{k} \vec{\varphi}$.

Before moving on to dimension 3, we first introduce some additional machinery which is the final technical ingredient required in order to make the induction to higher dimensions possible. Namely, for $\vec{f}, \vec{g}: \vec{x} \rightrightarrows \vec{y}$ parallel 1-cells, we define functors

$$
D(\vec{y})_{1}\left(\vartheta^{1} \vec{f}, y_{J}\right) \stackrel{\breve{H}_{\vec{f}, \vec{g}}^{1}}{\longrightarrow} D(\vec{y})_{1}\left(\Theta^{1} \vec{f}, y_{J}\right) \stackrel{H_{\vec{f}, \vec{g}}^{1}}{\longrightarrow} D(\vec{y})_{1}\left(\Theta^{1} \vec{f}, \vartheta^{1} \vec{g}\right)
$$

as follows:

$$
\begin{aligned}
& \breve{H}_{\vec{f}, \vec{g}}^{1}(-):=\left(-*_{0} \triangleleft \vec{f}\right), \\
& H_{\vec{f}, \vec{g}}^{1}(-):=\left(\triangleright \vec{g} *_{0}-\right) .
\end{aligned}
$$

With these functors at our disposal, we are in the position to make several remarks regarding their interaction with the other structures with which we are concerned. To begin with, when $\vec{\alpha}$ is a 2 -cell $\vec{f} \Rightarrow \vec{g}$,

$$
\begin{aligned}
& \triangleright \vec{\alpha}=H_{\vec{f}, \vec{g}}^{1}\left(\langle\vec{\alpha}\rangle_{\breve{x}_{d}}\right), \\
& \triangleleft \vec{\alpha}=\breve{H}_{\vec{f}, \vec{g}}^{1}\left(\langle\vec{y}\rangle\left(\breve{\alpha}_{d}\right)\right) .
\end{aligned}
$$

Also, these functors interact with $\vartheta^{2}$ in the sense that

$$
\breve{H}_{\vec{f}, \vec{g}}^{1}\left(\triangleright \vec{g} *_{0} \vartheta^{1}(\vec{\gamma})\right)=\vartheta_{\vec{f}, \vec{g}}^{2}(\vec{\gamma})=H_{\vec{f}, \vec{g}}^{1}\left(\vartheta^{1}(\vec{\gamma}) *_{0} \triangleleft \vec{f}\right) .
$$

In an informal sense, the problem of providing the elimination maps $\varphi_{J}$ will be seen to always amount, as above, to filling both a triangle and a square. In each case, the tactic is essentially the same as above and the functors $H^{k}$ and $\breve{H}^{k}$ allow us to express in the most perspicuous way the combinatorics of the situation for the squares and triangles, respectively. 
6.6. Elimination terms in dimension 3. In dimension 3 , given $\vec{\varphi}: \vec{\alpha} \Rightarrow \vec{\beta}$ a 3 -cell of $\int I_{A}$, we would like to describe the 3 -cells indicated in the following diagram:

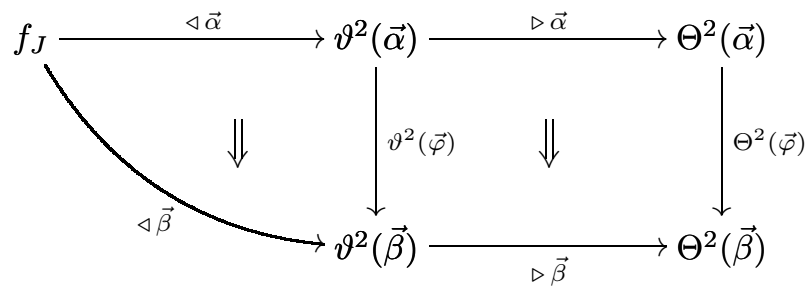

With this picture in mind we begin by defining, for fixed parallel 2-cells $\vec{\alpha}, \vec{\beta}: \vec{f} \rightrightarrows \vec{g}$, functors

$$
D(\vec{y})_{2}\left(f_{J}, \vartheta^{2} \vec{\beta}\right) \stackrel{\breve{H}_{\vec{\alpha}, \vec{\beta}}^{2}}{\longrightarrow} D(\vec{y})_{2}\left(f_{J}, \Theta^{2} \vec{\beta}\right) \stackrel{H_{\vec{\alpha}, \vec{\beta}}^{2}}{\longrightarrow} D(\vec{y})_{2}\left(\vartheta^{2} \vec{\alpha}, \Theta^{2} \vec{\beta}\right)
$$

as follows:

$$
\begin{aligned}
& \breve{H}_{\vec{\alpha}, \vec{\beta}}^{2}(-):=\triangleright \vec{\beta} *_{1} \breve{H}_{\vec{f}, \vec{g}}^{1}(-), \\
& H_{\vec{\alpha}, \vec{\beta}}^{2}(-):=H_{\vec{f}, \vec{g}}^{1}(-) *_{1} \triangleleft \vec{\alpha} .
\end{aligned}
$$

Next,

$$
\left(\int I_{A}\right)_{3}(\vec{\alpha}, \vec{\beta}) \stackrel{\vartheta_{\vec{\alpha}, \vec{\beta}}^{3}}{\longrightarrow} D(\vec{y})_{2}\left(f_{J}, \Theta^{2}(\vec{\beta})\right)
$$

is defined by

$$
\vartheta^{3}(-):=\triangleright(\vec{\beta}) *_{1} \vartheta^{2}(-) *_{1} \triangleleft(\vec{\alpha})
$$

Before going any further it is useful to establish several facts. First, we note that by a straightforward calculation:

$$
\vartheta_{\vec{f}, \vec{g}}^{2}(\vec{\gamma})=\breve{H}_{\vec{f}, \vec{g}}^{1}\left(\langle\vec{y}\rangle \widetilde{\Theta}^{2} \breve{\gamma}\right)
$$

We call (6.10) the triangle-law for dimension 2 and note that together with (6.7) it follows that the triangle from (6.9) may be filled with the 3-cell $\breve{H}_{\vec{f}, \vec{g}}^{1}\left(\langle\vec{y}\rangle \breve{\varphi}_{d}\right)$. Accordingly, we define

$$
\triangleright \vec{\varphi}:=\breve{H}_{\vec{\alpha}, \vec{\beta}}^{2}\left(\langle\vec{y}\rangle \breve{\varphi}_{d}\right) .
$$

Turning to the square, observe that

$$
\begin{aligned}
\Theta_{\vec{f}, \vec{g}}^{2}(\vec{\gamma}) *_{1} \triangleright \vec{\alpha} & =\left(g_{J} *_{0} \Theta^{1} \vec{\gamma}\right) *_{1}\left(\triangleright \vec{g} *_{0}\langle\vec{\alpha}\rangle_{\breve{x}_{d}}\right) \\
& =\left(\triangleright \vec{g} *_{0} \triangleleft \vec{g} *_{0} \Theta^{1} \vec{\gamma}\right) *_{1}\left(\triangleright \vec{g} *_{0}\langle\vec{\alpha}\rangle_{\breve{x}_{d}}\right) \\
& =\triangleright \vec{g} *_{0}\left(\left(\triangleleft \vec{g} *_{0} \Theta^{1} \vec{\gamma}\right) *_{1}\langle\vec{\alpha}\rangle_{\breve{x}_{d}}\right) \\
& =\triangleright \vec{g} *_{0} D\left(\nabla_{-}^{3} \vec{\gamma}\right)_{\breve{x}_{d}} .
\end{aligned}
$$

Consequently, we obtain the source square-law for dimension 2:

$$
\Theta_{\vec{f}, \vec{g}}^{2}(\vec{\gamma}) *_{1} \triangleright \vec{\alpha}=H_{\vec{f}, \vec{g}}^{1}\left(D\left(\nabla_{-}^{3} \vec{\gamma}\right)_{\breve{x}_{d}}\right) .
$$

Another straightforward calculation yields the target square-law for dimension 2:

$$
\triangleright \vec{\beta} *_{1} \vartheta_{\vec{f}, \vec{g}}^{2}(\vec{\gamma})=H_{\vec{f}, \vec{g}}^{1}\left(D\left(\nabla_{+}^{3} \vec{\gamma}\right)_{\breve{x}_{d}}\right) .
$$


Thus, the filler of the square in (6.9) is defined to be $H_{\vec{f}, \vec{g}}^{1}\left(\langle\vec{\varphi}\rangle_{\breve{x}_{d}}\right)$. Finally, we set

$$
\begin{aligned}
\triangleleft \vec{\varphi} & :=H_{\vec{\alpha}, \vec{\beta}}^{2}\left(\langle\vec{\varphi}\rangle_{\breve{x}_{d}}\right), \\
\varphi_{J} & :=\triangleright \vec{\varphi} *_{2} \triangleleft \vec{\varphi} .
\end{aligned}
$$

6.7. The construction in higher dimensions. Now, at higher dimensions, the construction of the elimination terms is by induction on dimension. In particular, we proceed by induction on $n \geq 2$ in such a way that at stage $(n+1)$-in addition to the existence of the required $(n+1)$-cells $\varphi_{J}$ - the following conditions are satisfied: I.e., .

(1) For all parallel $n$-cells $\vec{\alpha}, \vec{\beta}: \vec{f} \rightrightarrows \vec{g}$, there is a functor $\vartheta_{\vec{\alpha}, \vec{\beta}}^{n+1}$ parallel to $\Theta_{\vec{\alpha}, \vec{\beta}}^{n+1}$.

(2) For any $(n+1)$-cell $\vec{\varphi}: \vec{\alpha} \rightarrow \vec{\beta}$, there exist corresponding $(n+1)$-cells $\triangleright \vec{\varphi}$ and $\triangleleft \vec{\varphi}$ such that

$$
\alpha_{J} \stackrel{\triangleleft \vec{\varphi}}{\longrightarrow} \vartheta_{\vec{\alpha}, \vec{\beta}}^{n+1} \vec{\varphi} \stackrel{\triangleright \vec{\varphi}}{\longrightarrow} \Theta_{\vec{\alpha}, \vec{\beta}}^{n+1} \vec{\varphi}
$$

when $(n+1)$ is even, and

$$
\Theta_{\vec{\alpha}, \vec{\beta}}^{n+1} \vec{\varphi} \stackrel{\triangleleft \vec{\varphi}}{\longrightarrow} \vartheta_{\vec{\alpha}, \vec{\beta}}^{n+1} \vec{\varphi} \stackrel{\triangleright \vec{\varphi}}{\longrightarrow} \beta_{J}
$$

when $(n+1)$ is odd.

(3) There are, for $\vec{\alpha}$ and $\vec{\beta}$ parallel $n$-cells, functors $H_{\vec{\alpha}, \vec{\beta}}^{n}$ and $\breve{H}_{\vec{\alpha}, \vec{\beta}}^{n}$ such that

$$
\begin{array}{r}
D(\vec{y})_{n}\left(\vartheta^{n} \vec{\alpha}, g_{J}\right) \stackrel{\breve{H}_{\vec{\alpha}, \vec{\beta}}^{n}}{\longrightarrow} D(\vec{y})_{n}\left(\Theta^{n} \vec{\alpha}, g_{J}\right), \\
D(\vec{y})_{n}\left(\Theta^{n} \vec{\alpha}, \vartheta^{n} \vec{\beta}\right) \stackrel{H_{\vec{\alpha}, \vec{\beta}}^{n}}{\longrightarrow} D(\vec{y})_{n}\left(\Theta^{n} \vec{\alpha}, g_{J}\right)
\end{array}
$$

if $(n+1)$ is even; and

$$
\begin{array}{r}
D(\vec{y})_{n}\left(f_{J}, \vartheta^{n} \vec{\beta}\right) \stackrel{\breve{H}_{\vec{\alpha}, \vec{\beta}}^{n}}{\longrightarrow} D(\vec{y})_{n}\left(f_{J}, \Theta^{n} \vec{\beta}\right), \\
D(\vec{y})_{n}\left(\vartheta^{n} \vec{\alpha}, \Theta^{n} \vec{\beta}\right) \stackrel{H_{\vec{\alpha}, \vec{\beta}}^{n}}{\longrightarrow} D(\vec{y})_{n}\left(f_{J}, \Theta^{n} \vec{\beta}\right)
\end{array}
$$

if $(n+1)$ is odd.

(4) The following triangle-law is satisfied:

$$
\vartheta_{\vec{\alpha}, \vec{\beta}}^{n+1} \vec{\gamma}=\breve{H}_{\vec{\alpha}, \vec{\beta}}^{n}\left(\langle\vec{y}\rangle \widetilde{\Theta}^{n+1} \breve{\gamma}\right),
$$

when $\vec{\gamma}$ is any cell in the domain of $\vartheta^{n+1}$.

(5) If $\vec{\varphi}, \vec{\psi}: \vec{\alpha} \rightrightarrows \vec{\beta}$ are parallel $(n+1)$-cells, the following square-laws are satisfied:

$$
H_{\vec{\alpha}, \vec{\beta}}^{n}\left(D\left(\nabla_{-}^{n+2} \vec{\gamma}\right)_{\breve{x}_{d}}\right)= \begin{cases}\Theta^{n+1} \vec{\gamma} *_{n} \triangleright \vec{\varphi} & \text { if }(n+1) \text { is even, } \\ \vartheta^{n+1} \vec{\gamma} *_{n} \triangleleft \vec{\varphi} & \text { if }(n+1) \text { is odd; }\end{cases}
$$

and

$$
H_{\vec{\alpha}, \vec{\beta}}^{n}\left(D\left(\nabla_{+}^{n+2} \vec{\delta}\right)_{\breve{x}_{d}}\right)= \begin{cases}\triangleright \vec{\psi} *_{n} \vartheta^{n+1} \vec{\delta} & \text { if }(n+1) \text { is even, } \\ \triangleleft \vec{\psi} *_{n} \Theta^{n+1} \vec{\delta} & \text { if }(n+1) \text { is odd, }\end{cases}
$$

for appropriate cells $\vec{\gamma}$ and $\vec{\delta}$. Note that the $\nabla_{\xi}^{n+2}$ here are defined with respect to $\vec{\varphi}$ and $\vec{\psi}$. 
Assuming we have carried out the construction up to stage $n$, we claim that the following definitions at stage $(n+1)$ will satisfy the required conditions:

- For parallel $n$-cells $\vec{\alpha}$ and $\vec{\beta}$,

$$
\vartheta_{\vec{\alpha}, \vec{\beta}}^{n+1}(-):=\triangleright \vec{\alpha} *(n-1) \vartheta^{n}(-) *_{(n-1)} \triangleleft \vec{\beta} .
$$

- If $\vec{\alpha}$ and $\vec{\beta}$ are parallel $n$-cells $\vec{f} \rightrightarrows \vec{g}$, then we define

$$
H_{\vec{\alpha}, \vec{\beta}}^{n}(-):= \begin{cases}\triangleright \vec{\beta} *_{n} H_{\vec{f}, \vec{g}}^{n-1}(-) & \text { if }(n+1) \text { is even, } \\ H_{\vec{f}, \vec{g}}^{n-1}(-) *_{n} \triangleleft \vec{\alpha} & \text { if }(n+1) \text { is odd; }\end{cases}
$$

and

$$
\breve{H}_{\vec{\alpha}, \vec{\beta}}^{n}(-):= \begin{cases}\breve{H}_{\vec{f}, \vec{g}}^{n-1}(-) *_{n} \triangleleft \vec{\alpha} & \text { if }(n+1) \text { is even } \\ \triangleright \vec{\beta}_{n} \breve{H}_{\vec{f}, \vec{g}}^{n-1}(-) & \text { if }(n+1) \text { is odd. }\end{cases}
$$

- Given $\vec{\varphi}: \vec{\alpha} \Rightarrow \vec{\beta}$ a $(n+1)$-cell, we define

$$
\triangleleft \vec{\varphi}:= \begin{cases}\breve{H}_{\vec{\alpha}, \vec{\beta}}^{n}\left(\langle\vec{y}\rangle \breve{\varphi}_{d}\right) & \text { if }(n+1) \text { is even, } \\ H_{\vec{\alpha}, \vec{\beta}}^{n}\left(\langle\vec{\varphi}\rangle_{\breve{x}_{d}}\right) & \text { if }(n+1) \text { is odd; }\end{cases}
$$

and

$$
\triangleright \vec{\varphi}:= \begin{cases}H_{\vec{\alpha}, \vec{\beta}}^{n}\left(\langle\vec{\varphi}\rangle_{\breve{x}_{d}}\right) & \text { if }(n+1) \text { is even, } \\ \breve{H}_{\vec{\alpha}, \vec{\beta}}^{n}\left(\langle\vec{y}\rangle \breve{\varphi}_{d}\right) & \text { if }(n+1) \text { is odd. }\end{cases}
$$

- In all dimensions,

$$
\alpha_{J}:=\triangleright \vec{\alpha} *_{n} \triangleleft \vec{\alpha}
$$

when $\vec{\alpha}$ is a $(n+1)$-cell.

The reader can readily verify that we have already satisfied the conditions of the induction in the base case where $n=2$. We now turn to the induction step.

Lemma 6.4. With the definitions given above, the conditions of the construction are satisfied in all dimensions $(n+1)$.

Proof. First, assume $(n+1)$ is even with $n>2$ and let an $(n+1)$-cell $\vec{\varphi}: \vec{\alpha} \rightrightarrows \vec{\beta}$ in $\int I_{A}$ be given. Then, by the induction hypothesis and examination of the following diagram

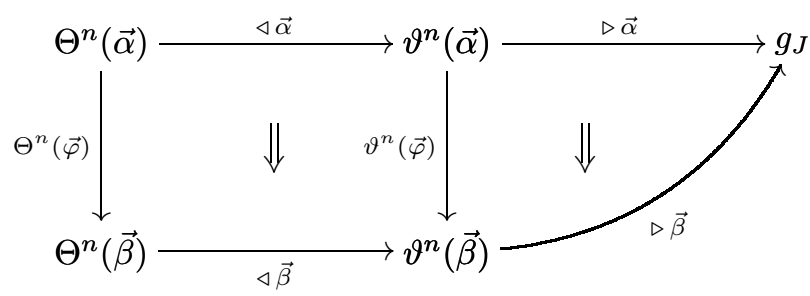

where $\vec{\alpha}, \vec{\beta}: \vec{f} \rightrightarrows \vec{g}$, it follows that conditions (1)-(3) are satisfied with the definitions given above. 
For the triangle law, we reason as follows:

$$
\begin{aligned}
\breve{H}_{\vec{\alpha}, \vec{\beta}}^{n}\left(\langle\vec{y}\rangle \widetilde{\Theta}^{n+1} \breve{\gamma}\right) & =\breve{H}_{\vec{f}, \vec{g}}^{n-1}\left(\langle\vec{y}\rangle \breve{\beta}_{d} *_{(n-1)}\langle\vec{y}\rangle \widetilde{\Theta}^{n} \breve{\gamma}\right) *{ }_{(n-1)} \triangleleft \vec{\alpha} \\
& =\breve{H}_{\vec{f}, \vec{g}}^{n-1}\left(\langle\vec{y}\rangle \breve{\beta}_{d}\right) *_{(n-1)} \breve{H}_{\vec{f}, \vec{g}}^{n-1}\left(\langle\vec{y}\rangle \widetilde{\Theta}^{n} \breve{\gamma}\right) *{ }_{(n-1)} \triangleleft \vec{\alpha} \\
& =\triangleright \vec{\beta} *_{(n-1)} \vartheta_{\vec{f}, \vec{g}}^{n}(\vec{\gamma}){ }_{(n-1)} \triangleleft \vec{\alpha} \\
& =\vartheta_{\vec{\alpha}, \vec{\beta}}^{n+1}(\vec{\gamma}),
\end{aligned}
$$

where the penultimate equation is by definition of $\triangleright \vec{\beta}$ and the induction hypothesis.

Next assume given $(n+1)$-cells $\vec{\varphi}, \vec{\psi}: \vec{\alpha} \rightrightarrows \vec{\beta}$. For the "source" square law, we have

$$
\begin{aligned}
& H_{\vec{\alpha}, \vec{\beta}}^{n}\left(D\left(\nabla_{-}^{n+2} \vec{\gamma}\right)_{\breve{x}_{d}}\right)=H_{\vec{\alpha}, \vec{\beta}}^{n}\left(D\left(\nabla_{+}^{n+1} \vec{\gamma}\right)_{\breve{x}_{d}} *_{n}\langle\vec{\varphi}\rangle_{\breve{x}_{d}}\right) \\
& =\left(\triangleright \vec{\beta} *_{(n-1)} H_{\vec{f}, \vec{g}}^{n-1}\left(D\left(\nabla_{+}^{n+1} \vec{\gamma}\right)\right)\right) *_{n} \triangleright \vec{\varphi} \\
& =\left(\triangleright \vec{\beta} *_{(n-1)} \triangleleft \vec{\beta} *_{(n-1)} \Theta^{n} \vec{\gamma}\right) *_{n} \triangleright \vec{\varphi} \\
& =\left(\beta_{J} *_{(n-1)} \Theta^{n} \vec{\gamma}\right) *_{n} \triangleright \vec{\varphi} \\
& =\Theta_{\vec{\alpha}, \vec{\beta}}^{n+1} \vec{\gamma} *_{n} \triangleright \vec{\varphi},
\end{aligned}
$$

where the third equation is by the induction hypothesis. For the "target" square law, we reason similarly and note that

$$
\begin{aligned}
H_{\vec{\alpha}, \vec{\beta}}^{n}\left(\langle\vec{\psi}\rangle_{\breve{x}_{d}} *_{n} D\left(\nabla_{-}^{n+1} \vec{\gamma}\right)_{\breve{x}_{d}}\right) & =\triangleright \vec{\psi} *_{n}\left(\triangleright \vec{\beta} *_{(n-1)} H_{\vec{f}, \vec{g}}^{n-1}\left(D\left(\nabla_{-}^{n+1} \vec{\gamma}\right)_{\breve{x}_{d}}\right)\right) \\
& =\triangleright \vec{\psi} *_{n}\left(\triangleright \vec{\beta} *_{(n-1)} \vartheta^{n} \vec{\gamma} *_{(n-1)} \triangleleft \vec{\alpha}\right) \\
& =\triangleright \vec{\psi} *_{n} \vartheta_{\vec{\alpha}, \vec{\beta}}^{n+1} \vec{\gamma},
\end{aligned}
$$

as required.

The induction step where $(n+1)$ is odd is essentially dual.

Using the lemma, we now have the following fundamental result.

Proposition 6.5. The cells of the form $\varphi_{J}$ constitute a section $J: \int I_{A} \rightarrow \int D$ of the projection map $\int D \rightarrow \int I_{A}$.

Proof. In light of Lemma 6.4 the only thing which remains is to verify that the assignment $(-)_{J}$ is functorial. This however is a consequence of the functoriality of $d$ and the construction of the "terms" $\varphi_{J}$ using the functors $H^{k}$ and $\breve{H}^{k}$.

\section{The main results}

With the machinery from the preceding sections at our disposal it is now possible to establish our main results.

7.1. Dependent sums and products. Before defining the dependent products and sums, we begin by describing some basic features of general setup. First, given a functor $A: \mathcal{C} \rightarrow \boldsymbol{\omega}$-Gpd we note that, by the basic properties of $\omega$-Gpd, there exists an $\omega$-groupoid denoted by $\Gamma(A)$ of sections of the projection $\int A \rightarrow \mathcal{C}$. 
I.e., the objects of $\Gamma(A)$ are sections

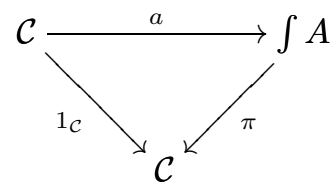

and arrows are 2-cells $\alpha: a \Rightarrow b$ for which $\pi \circ \alpha=1_{\mathcal{C}}$ and so forth at higher dimensions. Now, given a further functor $B: \int A \rightarrow \omega$-Gpd and an object $x$ of $\mathcal{C}$, we define a functor $\left\ulcorner B_{x}\right\urcorner: A_{x} \rightarrow \omega$-Gpd as follows:

Objects. Given an object $x_{-}$of $A_{x},\left\ulcorner B_{x}\right\urcorner\left(x_{-}\right)$is the $\omega$-groupoid $B\left(x, x_{-}\right)$.

1-Cells. Given an arrow $h: x_{-} \rightarrow x_{+}$in $A_{x}$, we define the functor $\left\ulcorner B_{x}\right\urcorner(h)$ : $B\left(x, x_{-}\right) \rightarrow B\left(x, x_{+}\right)$as follows:

$$
\left\ulcorner B_{x}\right\urcorner(h)(-):=B(x, h)(-) .
$$

Here we note that this is possible since $(x, h):\left(x, x_{-}\right) \rightarrow\left(x, x_{+}\right)$, where we have written $(x, h)$ instead of $\left(1_{x}, h\right)$.

$(\boldsymbol{n}+\mathbf{1})$-Cells Given a $(n+1)$-cell $\alpha: f \Rightarrow g$ in $A_{x}$ bounded by 0 -cells $x_{-}$and $x_{+}$, together with an object $y$ of $B\left(x, x_{-}\right)$, the transformation $\left\ulcorner B_{x}\right\urcorner(\alpha)$ is defined at $y$ by

$$
\left\ulcorner B_{x}\right\urcorner(\alpha)_{y}:=B(x, \alpha)_{y} .
$$

Now, we would like to describe, in this same setting, the dependent sum $\Sigma_{A, B}: \mathcal{C} \rightarrow$ $\omega$-Gpd. Note that, when $A$ and $B$ are apparent, we will often omit the subscripts.

Objects. Given an object $x$ of $\mathcal{C}$, we define

$$
\Sigma_{A, B}(x):=\int\left\ulcorner B_{x}\right\urcorner .
$$

Given $f: x \rightarrow y$ in $\mathcal{C}$ we would like to define $\Sigma_{A, B}(f): \int\left\ulcorner B_{x}\right\urcorner \rightarrow \int\left\ulcorner B_{y}\right\urcorner$, which we will write, for the sake of avoiding too many parentheses, as $\Sigma_{f}$. An object $\vec{v}$ of $\int\left\ulcorner B_{x}\right\urcorner$ is a pair $\left(v_{-}, v_{\sharp}\right)$ such that $v_{-}$is an object of $A_{x}$ and $v_{\sharp}$ is an object of $B\left(x, v_{-}\right)$. We adopt a similar notation in higher dimensions. We also write $\partial(x)^{n}$ for the weighted face functor of $\int\left\ulcorner B_{x}\right\urcorner$ and similarly for $\partial(y)^{n}$, etc. With this in mind we adopt the following definition (which we will discuss below):

1-Cells. Given $f: x \rightarrow y$ in $\mathcal{C}, \Sigma_{f}$ is defined on objects $\vec{v}$ of $\int\left\ulcorner B_{x}\right\urcorner$ by

$$
\Sigma_{f}(\vec{v}):=\left(v_{-} \cdot f, B\left(f, v_{-} \cdot f\right)\left(v_{\sharp}\right)\right),
$$

and on $(n+1)$-cells $\vec{\varphi}$ of $\int\left\ulcorner B_{x}\right\urcorner$ by

$$
\Sigma_{f}(\vec{\varphi}):=\left(\varphi_{-} \cdot f, B\left(f, w_{-} \cdot f\right)\left(\varphi_{\sharp}\right)\right),
$$

where $\vec{v}$ and $\vec{w}$ are the objects bounding $\vec{\varphi}$ at source and target position, respectively.

Note that this is a correct definition since, by functoriality of $B$, it is proved (simultaneously with the verification of the definitions) at each stage that

$$
B\left(f, w_{-} \cdot f\right)\left(\partial(x)_{\vec{\alpha}, \vec{\beta}}^{n+1}\left(\varphi_{-}\right)\right)=\partial(y)_{\Sigma_{f} \vec{\alpha}, \Sigma_{f} \vec{\beta}}^{n+1}\left(\varphi_{-} \cdot f\right),
$$

where $\vec{\alpha}$ and $\vec{\beta}$ are the $n$-cells bounding $\vec{\varphi}$.

In dimension $(n+1), \Sigma_{A, B}$ is given as follows: 
$(\boldsymbol{n}+\mathbf{1})$-Cells. Given a $(n+1)$-cell $\varphi: \alpha \rightarrow \beta$ in $\mathcal{C}, \Sigma_{\varphi}: \Sigma_{\alpha} \rightarrow \Sigma_{\beta}$ has as its component at the object $\vec{v}$ of $\int\left\ulcorner B_{x}\right\urcorner$ the $n$-cell

$$
\left(\Sigma_{\varphi}\right)_{\vec{v}}:=\left(v_{-} . \varphi, B\left(\varphi, v_{-} \cdot \varphi\right)\left(v_{\sharp}\right)\right)
$$

of $\int\left\ulcorner B_{y}\right\urcorner$.

To see that this makes sense, let $n$-cells $\lambda, \mu: \alpha \rightarrow \beta$ be given. Then it follows, by induction on $n$ that,

$$
\partial(y)_{\left(\Sigma_{\lambda}\right)_{\vec{v}},\left(\Sigma_{\mu}\right)_{\vec{v}}}^{(n+1)}\left(v_{-} \cdot \gamma\right)= \begin{cases}B\left(\mu, v_{-} \cdot \gamma\right)_{v_{\sharp}} & \text { if }(n+1) \text { is even, } \\ B\left(\lambda, v_{-} \cdot \gamma\right)_{v_{\sharp}} & \text { if }(n+1) \text { is odd, }\end{cases}
$$

where $\gamma$ is any $m$-cell with $m \geq n+1$ bounded by $\lambda$ and $\mu$ (i.e., $s^{m-n} \gamma=\lambda$ and $t^{m-n} \gamma=\mu$ ). This is a straightforward induction using functoriality of $B$. This completes the description of the interpretation $\Sigma_{A, B}$ of dependent sums in this setting. With these definitions, the verification of functoriality is a routine calculation.

Next, we define the dependent product $\Pi_{A, B}: \mathcal{C} \rightarrow \omega$-Gpd to be the functor which sends an object $x$ to the $\omega$-groupoid $\Gamma\left(\left\ulcorner B_{x}\right\urcorner\right)$ of sections of the projection $\int\left\ulcorner B_{x}\right\urcorner \rightarrow A_{x}$. Explicitly, $\Pi_{A, B}$ is as follows:

Objects. $\Pi_{A, B}(x)$ is defined to be $\Gamma\left(\left\ulcorner B_{x}\right\urcorner\right)$.

1-Cells. Given an arrow $f: x \rightarrow y$ in $\mathcal{C}, \Pi_{A, B}(f)$ is the functor sending a section $a$ in $\Gamma\left(\left\ulcorner B_{x}\right\urcorner\right)$ to the section $\Sigma_{f} \circ a \circ A_{f^{-1}}$. In general, $\Pi_{A, B}(f)$ sends an arbitrary cell $\varphi$ of $\Gamma\left(\left\ulcorner B_{x}\right\urcorner\right)$ to the composite $\Sigma_{f} \circ \varphi \circ A_{f^{-1}}$ as indicated in the following diagram:

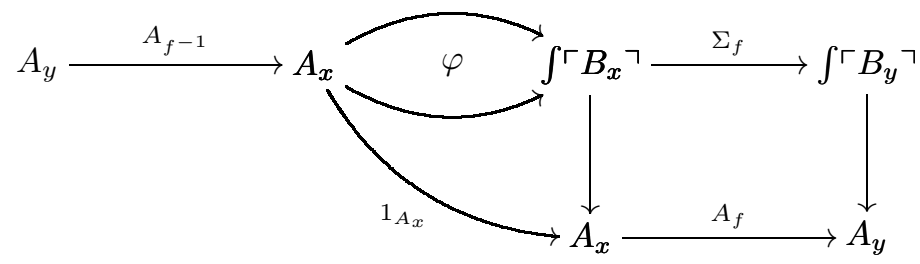

$(\boldsymbol{n}+\mathbf{1})$-Cells. In general, when $\gamma: \alpha \rightarrow \beta$ is a $(n+1)$-cell in $\mathcal{C}$ and $a$ is an object of $\Pi_{A, B}(x)$, the dependent product is defined by setting

$$
\Pi_{A, B}(\gamma)_{a}:=\Sigma_{\gamma} \circ a \circ A_{\sigma(\gamma)},
$$

where $\sigma: \mathcal{C} \rightarrow \mathcal{C}^{\text {op }}$ is the dual functor for $\mathcal{C}$ as described in Section 4 .

This completes the description of the dependent sums and products. The introduction and elimination terms are then obtained as generalizations of those given in $[9]$.

7.2. Results. With the interpretations given we obtain the following result extending the original groupoids model from ibid. to the setting of $\omega$-groupoids:

Theorem 7.1. The interpretation given above soundly interprets the basic form of intensional type theory with dependent sums, dependent products and identity types (i.e., the theory $\mathbb{T}_{\omega}$ from [1]). Moreover, the $\eta$-rules for dependent sums and products are also satisfied.

PROOF. Using the interpretation given above it is straightforward to verify that all of the required laws, including all of the Beck-Chevalley laws, are satisfied. 
Recall from $[1,27]$ that we employ the following notation for iterated identity types:

$$
\underline{A}^{n+1}\left(a_{1}, b_{1} ; \ldots ; a_{n+1}, b_{n+1}\right):=\underline{\underline{A^{n}}\left(a_{1}, b_{1} ; \ldots ; a_{n}, b_{n}\right)}\left(a_{n+1}, b_{n+1}\right) .
$$

In this notation, the principle of $n$-dimensional uniqueness of identity proofs is

$$
\frac{\vdash a_{n+1}, b_{n+1}: \underline{A}^{n}\left(a_{1}, b_{1} ; \ldots ; a_{n}, b_{n}\right)}{\vdash a_{n+1}=b_{n+1}: \underline{A}^{n}\left(a_{1}, b_{1} ; \ldots ; a_{n}, b_{n}\right)}
$$

It is a trivial consequence of the interpretation that, for all $n \geq 1$, the $\omega$-groupoid model refutes $\operatorname{UIP}_{n}$. To see this, form the globular set $G$ which is described as follows:

0-Cells. Two 0-cells $\perp^{0}$ and $\top^{0}$.

$(\boldsymbol{n}+\mathbf{1})$-Cells. Two parallel $(n+1)$-cells $\perp^{n+1}, \top^{n+1}: \perp^{n} \rightarrow \top^{n}$.

Then the free $\omega$-groupoid on $G$ refutes uniqueness of identity proofs at all dimensions (in both the forms which deal with definitional and propositional equality). This is the case because the terms of identity types in any type interpreted as this free groupoid are simply the cells of this groupoid. I.e., we have:

Corollary 7.2. For all $n \geq 1$, the principle $\mathrm{UIP}_{n}$ is not derivable in $\mathbb{T}_{\omega}$. Similar, the principle of uniqueness of identity proofs with respect to propositional equality is also not derivable.

Recall from [1] that the $n$-truncation rule is as follows:

$$
\frac{\vdash a_{n+1}, b_{n+1}: \underline{A}^{n}\left(a_{1}, b_{1} ; \ldots ; a_{n}, b_{n}\right) \quad \vdash p: \underline{A}^{n+1}\left(a_{1}, b_{1} ; \ldots ; a_{n+1}, b_{n+1}\right)}{\vdash a_{n+1}=b_{n+1}: \underline{A}^{n}\left(a_{1}, b_{1} ; \ldots ; a_{n}, b_{n}\right)} \mathrm{TR}_{n}
$$

Our main corollary of Theorem 7.1 then states that the identity types in intensional type theory are nontrivial in all dimensions in the sense that all of the truncation rules are refuted by the $\omega$-groupoid model. The same counterexample from above suffices to give this result as well. I.e., we have:

Corollary 7.3. For $n \geq 0, \mathrm{TR}_{n}$ is not derivable in $\mathbb{T}_{\omega}$.

We note that all of these results also apply in the case of type theory with the added $\eta$-rules for dependent products and sums, since these rules are valid in the model.

\section{Appendix A. Rules governing identity types, dependent products and dependent sums}

In this appendix we describe the syntax of the system considered in this paper (called $\mathbb{T}_{\omega}$ in $[1]$ ). All rules below are stated in an ambient context which is omitted for ease of presentation.

\section{A.1. Identity types.}

$$
\begin{aligned}
& \frac{a, b: A}{\vdash \underline{A}(a, b): \text { type }} \text { formation } \\
& \frac{a: A}{r(a): \underline{A}(a, a)} \text { introduction }
\end{aligned}
$$




$$
\begin{gathered}
x: A, y: A, z: \underline{A}(x, y) \vdash B(x, y, z): \text { type } \\
x: A \vdash \varphi(x): B(x, x, r(x)) \\
\frac{f: \underline{A}(a, b)}{\mathrm{J}(\varphi(x), a, b, f): B(a, b, f)} \text { elimination } \\
\frac{a: A}{\mathrm{~J}(\varphi(x), a, a, r(a))=\varphi(a): B(a, a, r(a))} \text { conversion }
\end{gathered}
$$

\section{A.2. Dependent products.}

$$
\begin{gathered}
\frac{x: A \vdash B(x): \text { type }}{\prod_{x: A} B(x): \text { type }} \prod \text { formation } \\
\frac{x: A \vdash f(x): B(x)}{\lambda_{x: A} f(x): \prod_{x: A} B(x)} \prod \text { introduction } \\
\frac{f: \prod_{x: A} B(x) \quad a: A}{\operatorname{app}(f, a): B(a) \cdot} \prod \text { elimination } \\
\frac{\lambda_{x: A} f(x): \prod_{x: A} B(x)}{\operatorname{app}\left(\lambda_{x: A} f(x), a\right)=f(a): B(a)} \prod \text { conversion }
\end{gathered}
$$

\section{A.3. Dependent sums.}

$$
\begin{gathered}
\frac{x: A \vdash B(x): \text { type }}{\sum_{x: A} B(x): \text { type }} \sum \text { formation } \\
\frac{a: A \quad b: B(a)}{\operatorname{pair}(a, b): \sum_{x: A} B(x)} \sum \text { introduction } \\
\frac{x: A, y: B(x) \vdash \psi(x, y): C(\operatorname{pair}(x, y))}{p: \sum_{x: A} B(x) \quad} \sum \text { elimination } \\
\frac{\mathrm{R}(\psi(x, y), p): C(p)}{a: A \quad b: B(a)} \quad x, y: B(x) \vdash \psi(x, y): C(\operatorname{pair}(x, y)) \\
\mathrm{R}(\psi(x, y), \operatorname{pair}(a, b))=\psi(a, b): C(\operatorname{pair}(a, b))
\end{gathered}
$$

\section{Appendix B. The universal property of the Grothendieck construction}

In this appendix we provide a detailed proof that the Grothendieck construction $\int A$ for strict $\omega$-categories introduced in Section 3 can be described as the coend $\int^{x}(x \downarrow \mathcal{C}) \times A_{x}$. First we turn to a more detailed description of the comma $\omega$ category $(x \downarrow \mathcal{C})$ when $x$ is a 0 -cell of an $\omega$-category $\mathcal{C}$. 
B.1. The comma $\omega$-category. Given an $\omega$-category $\mathcal{C}$ and an object $x$ of $\mathcal{C}$ the comma $\omega$-category $(x \downarrow \mathcal{C})$ will be constructed inductively in such a way that at each stage a $n$-cell of $(x \downarrow \mathcal{C})$ is a pair $\left(f, f_{-}\right)$such that $f$ is a $n$-cell of $\mathcal{C}$ and $f_{-}$is a $(n+1)$-cell of $\mathcal{C}$. At each stage it will also be necessary to construct auxiliary functors which describe the boundaries of the cells $f_{-}$in terms of $f$ and lower dimensional cells. These are like the weighted face functors discussed in the construction of the Grothendieck construction given above and we denote them also using the notation $\mathfrak{d}$. Because we will not require the combinatorial details of the comma category $(x \downarrow \mathcal{C})$ we will merely describe the different cells and leave the proof that this yields an $\omega$-category to the reader. We mention that slicing constructions in the higher-dimensional setting have been studied in recent work by Palm [20].

0-Cells. A 0 -cell of $(x \downarrow \mathcal{C})$ is a pair $\vec{y}=\left(y, y_{-}\right)$such that $y$ is an object of $\mathcal{C}$ and $y_{-}$is an arrow $x \rightarrow y$ in $\mathcal{C}$. For fixed 0 -cells $\vec{y}, \vec{z}$ define $\partial_{\vec{y}, \vec{z}}(-):=-*_{0} y_{-}$. When $\vec{y}, \vec{z}$ are understood the subscript will be omitted.

1-Cells. A 1-cell $\vec{y} \rightarrow \vec{z}$ of $(x \downarrow \mathcal{C})$ is a tuple $\vec{f}=\left(f, f_{-}\right)$with $f: y \rightarrow z$ a 1-cell in $\mathcal{C}$ and $f_{-}: \partial(f) \rightarrow z_{-}$a 2-cell in $\mathcal{C}$. Fixing parallel 1-cells $\vec{f}, \vec{g}: \vec{y} \rightarrow \vec{z}$, we define $\partial_{\vec{f}, \vec{g}}(-):=g_{-} *_{1} \partial(-)$.

$(\boldsymbol{n}+\mathbf{1})$-Cells for $(\boldsymbol{n}+\mathbf{1})$ even. A $(n+1)$-cell $\vec{\varphi}: \vec{\gamma} \rightarrow \vec{\delta}$ is given by a $(n+1)$ cell $\varphi: \gamma \rightarrow \delta$ in $\mathcal{C}$ and a $(n+2)$-cell $\varphi_{-}: \gamma_{-} \rightarrow \partial(\varphi)$ in $\mathcal{C}$. Given parallel $(n+1)$-cells $\vec{\varphi}$ and $\vec{\psi}$ we define $\partial_{\vec{\varphi}, \vec{\psi}}(-):=\partial(-) *_{n+1} \varphi_{-}$.

$(\boldsymbol{n}+\mathbf{1})$-Cells for $(\boldsymbol{n}+\mathbf{1})$ odd. A $(n+1)$-cell $\vec{\varphi}: \vec{\gamma} \rightarrow \vec{\delta}$ is given by a $(n+1)$ cell $\varphi: \gamma \rightarrow \delta$ in $\mathcal{C}$ and a $(n+2)$-cell $\varphi_{-}: \partial(\varphi) \rightarrow \delta_{-}$in $\mathcal{C}$. Given parallel $(n+1)$-cells $\vec{\varphi}$ and $\vec{\psi}$ we define $\partial_{\vec{\varphi}, \vec{\psi}}(-):=\psi_{-} *_{n+1} \partial(-)$.

Here we use the notation $\partial$ instead of $\mathfrak{d}$ to describe the required "weighted boundaries" of the construction since we will be mixing both the Grothendieck construction and its $\mathfrak{d}$ and comma categories in Section B.2 below.

Allowing $x$ to vary we obtain a functor (of $\omega$-categories) $(-\downarrow \mathcal{C}): \mathcal{C}^{\text {op }} \rightarrow \omega$-Cat which we describe as follows.

Dimension 1. Given an arrow $l: v \rightarrow x$ in $\mathcal{C}^{\text {op }}$ we have a functor $l^{*}:(x \downarrow \mathcal{C}) \rightarrow$ $(v \downarrow \mathcal{C})$ which sends a cell $\vec{\varphi}=\left(\varphi, \varphi_{-}\right)$of any dimension of $(x \downarrow \mathcal{C})$ to

$$
l^{*}(\vec{\varphi}):=\left(\varphi, \varphi_{-} *_{0} l\right) .
$$

This is trivially functorial.

Dimension 2. Given parallel arrows $l, m: v \rightarrow x$ in $\mathcal{C}$ and a 2-cell $\lambda: l \rightarrow m$ the induced transformation $\lambda^{*}: l^{*} \rightarrow m^{*}$ has as its component at an object $\vec{y}=$ $\left(y, y_{-}\right)$of $(x \downarrow \mathcal{C})$ the cell

$$
\left(\lambda^{*}\right)_{\vec{y}}:=\left(1_{y}, y_{-} *_{0} \lambda\right) .
$$

With this definition the naturality condition for natural transformations of $\omega$ categories is straightforward.

Dimension $\boldsymbol{n}>2$. Given parallel $(n-1)$-cells $\alpha$ and $\beta$ in $\mathcal{C}$ (bounded by $v$ and $x$, respectively, at dimension 0 ), and a $n$-cell $\varphi: \alpha \rightarrow \beta$, the component of the induced transformation $\varphi^{*}: \alpha^{*} \rightarrow \beta^{*}$ at an object $\vec{y}$ of $(x \downarrow \mathcal{C})$ is given by $\left(1_{y}, y_{-} * \varphi\right)$. This clearly satisfies the naturality condition.

This process is clearly functorial (since it is just given by precomposition). 
B.2. Description of the coend inclusions. We now turn to the description of the coend inclusion functors $\iota_{x}:(x \downarrow \mathcal{C}) \times A_{x} \rightarrow \int A$, for $A: \mathcal{C} \rightarrow \omega$-Cat. Again, $\iota_{x}$ is given by an inductive construction where we must verify certain equations at each stage. We will begin with the first few dimensions and then we will explain precisely the conditions of the induction.

0-Cells. A 0 -cell of $(x \downarrow \mathcal{C}) \times A_{x}$ is a tuple $\vec{y}=\left(y, y_{-}, y_{o}\right)$ such that $y$ is a 0 -cell of $\mathcal{C}, y_{-}$is a 1-cell $y_{-}: x \rightarrow y$ in $\mathcal{C}$, and $y_{o}$ is an object of $A_{x}$. We define

$$
\iota_{x}(\vec{y}):=\left(y, y_{o} \cdot y_{-}\right)
$$

So, in particular, $\iota_{x}(\vec{y})_{-}=y_{0} \cdot y_{-}$.

Now, given two 0-cells $\vec{y}$ and $\vec{z}$ of $(x \downarrow \mathcal{C}) \times A_{x}$ we have the weighted boundary functors $\partial_{\vec{y}, \vec{z}}(-)$ (for $(x \downarrow \mathcal{C})$ ) and $\mathfrak{d}_{\iota_{x}(\vec{y}), \iota_{x}(\vec{z})}(-)$ (for $\int A$ ). These two functors are moreover related by the law

$$
\mathfrak{d}_{\iota_{x}(\vec{y}), \iota_{x}(\vec{z})}(-)=y_{0} \cdot \partial_{\vec{y}, \vec{z}}(-)
$$

since

$$
\mathfrak{d}_{\iota_{x}(\vec{y}), \iota_{x}(\vec{z})}(-)=y_{o} \cdot y_{-} \cdot-=y_{0} \cdot\left(-*_{0} y_{-}\right)=y_{0} \cdot \partial_{\vec{y}, \vec{z}}(-) .
$$

1-Cells. A 1-cell $\vec{f}: \vec{y} \rightarrow \vec{z}$ of $(x \downarrow \mathcal{C}) \times A_{x}$ is a tuple $\vec{f}=\left(f, f_{-}, f_{o}\right)$ such that $f$ is a 1-cell $f: y \rightarrow z$ in $\mathcal{C}, f_{-}: \partial_{\vec{y}, \vec{z}}(f) \rightarrow z_{-}$is a 2-cell in $\mathcal{C}$, and $f_{o}: y_{o} \rightarrow z_{o}$ is a 1-cell in $A_{x}$. Then $\iota_{x}(\vec{f})$ is defined to be the pair $\left(f, \iota_{x}(\vec{f})_{-}\right)$where $\iota_{x}(\vec{f})_{-}$is the 1-cell

$$
\mathfrak{d}_{\iota_{x}(\vec{y}), \iota_{x}(\vec{z})}(f)=y_{o} \cdot \partial_{\vec{y}, \vec{z}}(f) \stackrel{y_{o} \cdot f_{-}}{\longrightarrow} y_{o} \cdot z_{-} \stackrel{f_{o} \cdot z_{-}}{\longrightarrow} z_{o} \cdot z_{-},
$$

where the equation is by (B.1). I.e.,

$$
\iota_{x}(\vec{f})_{-}:=\left(f_{o} \cdot z_{-}\right) *_{0}\left(y_{o} \cdot f_{-}\right) .
$$

Now, before moving on to the next stage we observe that, for 1-cells $\vec{f}, \vec{g}$ of $(x \downarrow$ $\mathcal{C}) \times A_{x}$, the following equation relating the weighted boundary functors holds:

$$
\mathfrak{d}_{\iota_{x}(\vec{f}), \iota_{x}(\vec{g})}(-)=\left(g_{o} \cdot z_{-}\right) *_{0}\left(y_{o} \cdot \partial_{\vec{f}, \vec{g}}(-)\right)
$$

since

$$
\begin{aligned}
\mathfrak{d}_{\iota_{x}(\vec{f}), \iota_{x}(\vec{g})}(-) & =\iota_{x}(\vec{g})_{-} *_{0} \mathfrak{d}_{\iota_{x}(\vec{y}), \iota_{x}(\vec{z})}(-) \\
& =\left(\left(g_{0} \cdot z_{-}\right) *_{0}\left(y_{o} \cdot g_{-}\right)\right) *_{0}\left(y_{o} \cdot \partial_{\vec{y}, \vec{z}}(-)\right) \\
& =\left(g_{0} \cdot z_{-}\right) *_{0}\left(y_{0} \cdot\left(g_{-} *_{1} \partial_{\vec{y}, \vec{z}}(-)\right)\right) \\
& =\left(g_{0} \cdot z_{-}\right) *_{0}\left(y_{0} \cdot \partial_{\vec{f}, \vec{g}}(-)\right),
\end{aligned}
$$

where the first equation is by definition of $\partial_{\iota_{x}(\vec{f}), \iota_{x}(\vec{g})}(-)$, the second equation is by (B.1), the third equation is by associativity of $*_{0}$ and functoriality of $A$, and the fourth equation is by definition of $\partial_{\vec{f}, \vec{g}}(-)$.

2-Cells. A 2-cell $\vec{\alpha}: \vec{f} \rightarrow \vec{g}$ in $(x \downarrow \mathcal{C}) \times A_{x}$ consists of a tuple $\vec{\alpha}=\left(\alpha, \alpha_{-}, \alpha_{o}\right)$ such that $\alpha: f \rightarrow g$ is a 2-cell in $\mathcal{C}, \alpha_{-}$is a 3-cell $\alpha_{-}: f_{-} \rightarrow \partial_{\vec{f}, \vec{g}}(\alpha)$ in $\mathcal{C}$, and $\alpha_{o}$ is a 2-cell $\alpha_{o}: f_{o} \rightarrow g_{o}$ in $A_{x}$. Then $\iota_{x}(\vec{\alpha})$ is a pair $\left(\alpha, \iota_{x}(\vec{\alpha})_{-}\right)$where $\iota_{x}(\vec{\alpha})_{-}$is the 
2-cell

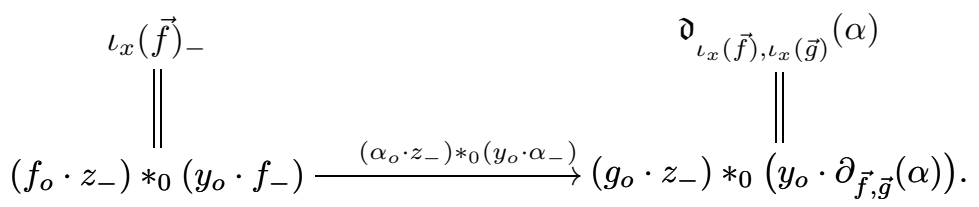

I.e.,

$$
\iota_{x}(\vec{\alpha})_{-}:=\left(\alpha_{o} \cdot z_{-}\right) *_{0}\left(y_{o} \cdot \alpha_{-}\right) .
$$

Again, for 2-cells $\vec{\alpha}$ and $\vec{\beta}$ of $(x \downarrow \mathcal{C}) \times A_{x}$, there is an equation

$$
\mathfrak{d}_{\iota_{x}(\vec{\alpha}), \iota_{x}(\vec{\beta})}(-)=\left(\alpha_{o} \cdot z_{-}\right) *_{0}\left(y_{o} \cdot \partial_{\vec{\alpha}, \vec{\beta}}(-)\right)
$$

relating the weighted boundary functors. This equation is obtained as follows:

$$
\begin{aligned}
\mathfrak{d}_{\iota_{x}(\vec{\alpha}), \iota_{x}(\vec{\beta})}(-) & =\mathfrak{d}_{\iota_{x}(\vec{f}), \iota_{x}(\vec{g})}(-) *_{1} \iota_{x}(\vec{\alpha})_{-} \\
& =\left(\left(g_{o} \cdot z_{-}\right) *_{0}\left(y_{o} \cdot \partial_{\vec{f}, \vec{g}}(-)\right) *_{1}\left(\left(\alpha_{o} \cdot z_{-}\right) *_{0}\left(y_{o} \cdot \alpha_{-}\right)\right)\right. \\
& =\left(\alpha_{o} \cdot z_{-}\right) *_{0}\left(y_{o} \cdot\left(\partial_{\vec{f}, \vec{g}}(-) *_{2} \alpha_{-}\right)\right) \\
& =\left(\alpha_{o} \cdot z_{-}\right) *_{0}\left(y_{o} \cdot \partial_{\vec{\alpha}, \vec{\beta}}(-)\right),
\end{aligned}
$$

where the penultimate equation is by the interchange law and functoriality of $A$.

3-Cells. A 3-cell $\vec{\varphi}: \vec{\alpha} \rightarrow \vec{\beta}$ of $(x \downarrow \mathcal{C}) \times A_{x}$ is a tuple $\vec{\varphi}=\left(\varphi, \varphi_{-}, \varphi_{o}\right)$ such that $\varphi: \alpha \rightarrow \beta$ is a 3 -cell in $\mathcal{C}, \varphi_{-}$is a 4 -cell $\varphi_{-}: \partial_{\vec{\alpha}, \vec{\beta}}(\varphi) \rightarrow \beta_{-}$in $\mathcal{C}$, and $\varphi_{o}: \alpha_{o} \rightarrow \beta_{o}$ is a 3 -cell in $A_{x}$. Then $\iota_{x}(\vec{\varphi})$ is the pair $\left(\varphi, \iota_{x}(\vec{\varphi})_{-}\right)$where $\iota_{x}(\vec{\varphi})_{-}$is the 3-cell

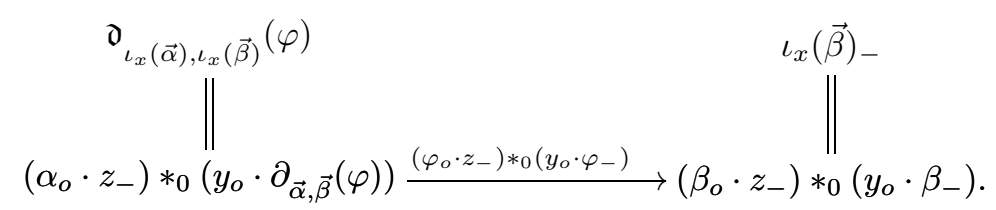

in $A_{z}$. I.e.,

$$
\iota_{x}(\vec{\varphi})_{-}:=\left(\varphi_{o} \cdot z_{-}\right) *_{0}\left(y_{0} \cdot \varphi_{-}\right) .
$$

Now, given two 3 -cells $\vec{\varphi}, \vec{\psi}: \vec{\alpha} \rightarrow \vec{\beta}$ in $(x \downarrow \mathcal{C}) \times A_{x}$, we have the equation

$$
\mathfrak{d}_{\iota_{x}(\vec{\varphi}), \iota_{x}(\vec{\psi})}(-)=\left(\psi_{o} \cdot z_{-}\right) *_{0}\left(y_{o} \cdot \partial_{\vec{\varphi}, \vec{\psi}}(-)\right),
$$

since

$$
\begin{aligned}
\mathfrak{d}_{\iota_{x}(\vec{\varphi}), \iota_{x}(\vec{\psi})}(-) & =\iota_{x}(\vec{\psi})_{-} *_{2} \mathfrak{d}_{\iota_{x}(\vec{\alpha}), \iota_{x}(\vec{\beta})}(-) \\
& =\left(\left(\psi_{o} \cdot z_{-}\right) *_{0}\left(y_{o} \cdot \psi_{-}\right)\right) *_{2}\left(\left(\alpha_{o} \cdot z_{-}\right) *_{0}\left(y_{o} \cdot \partial_{\vec{\alpha}, \vec{\beta}}(-)\right)\right) \\
& =\left(\psi_{o} \cdot z_{-}\right) *_{0}\left(y_{o} \cdot\left(\psi_{-} *_{3} \partial_{\vec{\alpha}, \vec{\beta}}(-)\right)\right) \\
& =\left(\psi_{o} \cdot z_{-}\right) *_{0}\left(y_{o} \cdot \partial_{\vec{\varphi}, \vec{\psi}}(-)\right)
\end{aligned}
$$

Indeed, as suggested by these cases, we define at all subsequent stages of the construction

$$
\iota_{x}(\vec{\xi})_{-}:=\left(\xi_{o} \cdot z_{-}\right) *_{0}\left(y_{o} \cdot \xi_{-}\right),
$$

and that we must verify that the equation

$$
\mathfrak{d}_{\iota_{x}(\vec{\xi}), \iota_{x}(\vec{\zeta})}(-)=\left(\zeta_{o} \cdot z_{-}\right) *_{0}\left(y_{o} \cdot \partial_{\vec{\xi}, \vec{\zeta}}(-)\right.
$$


holds. However, the verification that this equation holds generalizes directly from the cases of dimensions 2 and 3 given above (since the definitions of $\mathfrak{d}$ and $\partial$ also generalize directly from these cases). That $\iota_{x}$ is functorial is straightforward and is left to the reader.

The functors $\iota_{x}$, for $x$ an object of $\mathcal{C}$, constitute a dinatural transformation $(-\downarrow \mathcal{C}) \times A_{-} \rightarrow \int A$ in the sense that, for any $l, m: v \rightarrow x$ in $\mathcal{C}$ and any $n$-cell $\varphi$ bounded by $l$ and $m$ (including the degenerate case where $l=m=\varphi$ ), the diagram

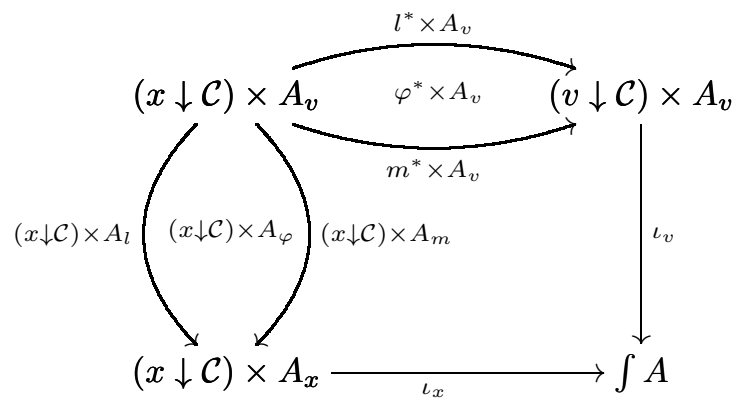

in $\omega$-Cat commutes. To see this note that, for 0 -cells $\vec{y}=\left(y, y_{-}, y_{o}\right)$ of $(x \downarrow \mathcal{C}) \times A_{v}$,

$$
\iota_{v} \circ\left(l^{*} \times A_{v}\right)(\vec{y})=\left(y, y_{o} \cdot\left(y_{-} *_{0} l\right)\right)=\left(y, y_{o} \cdot l \cdot y_{-}\right)=\iota_{x} \circ\left((x \downarrow \mathcal{C}) \times A_{l}\right)(\vec{y}),
$$

and, for $\vec{\varphi}$ any higher dimensional cell bounded by 0 -cells $\vec{y}$ and $\vec{z}$, we have

$$
\begin{aligned}
\iota_{v} \circ\left(l^{*} \times A_{v}\right)(\vec{\varphi}) & =\left(\varphi,\left(\varphi_{o} \cdot\left(z_{-} *_{0} l\right)\right) *_{0}\left(y_{o} \cdot\left(\varphi_{-} *_{0} l\right)\right)\right) \\
& =\left(\varphi,\left(\varphi_{o} \cdot l \cdot z_{-}\right) *_{0}\left(y_{o} \cdot l \cdot \varphi_{-}\right)\right) \\
& =\iota_{x} \circ\left((x \downarrow \mathcal{C}) \times A_{l}\right)(\vec{\varphi}) .
\end{aligned}
$$

B.3. Verification of the universal property. We will now show that the $\iota_{-}$constitute the universal dinatural transformation of this form. I.e., we prove that if $\mathcal{A}$ is any $\omega$-category together with a family of dinatural transformations (in the $\omega$-categorical sense of a $\omega$-categorical natural transformation) $\xi:(-\downarrow \mathcal{C}) \times A_{-} \rightarrow \mathcal{A}$, then there exists a canonical functor (of $\omega$-categories) $F: \int A \rightarrow \mathcal{A}$ such that

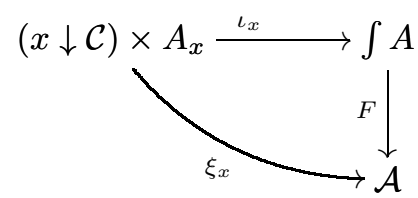

commutes for every object $x$ of $\mathcal{C}$. The construction of $F$ is a fairly involved induction on dimension. In particular, we will need to construct, for each pair of $n$-cells $\vec{\alpha}, \vec{\beta}$, auxiliary functors $b_{\vec{\alpha}, \vec{\beta}}(-,-)$ and $\sharp_{\vec{\alpha}, \vec{\beta}}(-)$ (the domains and codomains of these functors will be clear from the description given below). Moreover, we will prove at each stage that

$$
\sharp_{\vec{\alpha}, \vec{\beta}}\left(\mathfrak{d}_{\vec{\alpha}, \vec{\beta}}(-)\right)= \begin{cases}b_{\vec{\alpha}, \vec{\beta}}(\beta,-) & \text { if } n \text { is even, } \\ b_{\vec{\alpha}, \vec{\beta}}(\alpha,-) & \text { if } n \text { is odd. }\end{cases}
$$

Additionally, we will require that at even stages $F(\vec{\alpha})=b_{\vec{\alpha}, \vec{\beta}}\left(\alpha, 1_{\alpha}\right)$ and $F(\vec{\beta})=$ $\sharp_{\vec{\alpha}, \vec{\beta}}\left(\beta_{-}\right)$, and that the dual equations hold at odd stages. The final fact we require is that, as arrows in $(y \downarrow \mathcal{C})$, if $\varphi: \alpha \rightarrow \beta$ is an $n$-cell in $\mathcal{C}$ (bounded by 0 -cells $x$ and $y)$, then $\left(\varphi, 1_{\varphi}\right):\left(\alpha, 1_{\alpha}\right) \rightarrow(\beta, \varphi)$ when $n$ is odd. Similarly, when $n$ is 
even, $\left(\varphi, 1_{\varphi}\right):(\alpha, \varphi) \rightarrow\left(\beta, 1_{\beta}\right)$. This last fact is a straightforward induction, using the definition of the $\omega$-category $(y \downarrow \mathcal{C})$. The second condition will be trivially satisfied by definition in each case and therefore we omit explicit mention of it in the construction below. We will also omit the proof of functoriality of the auxiliary functors as this is also straightforward.

We describe $F$ as follows:

0-Cells. Given a 0 -cell $\vec{x}=\left(x, x_{-}\right)$of $\int A$, we define

$$
F(\vec{x}):=\xi_{x}\left(x, 1_{x}, x_{-}\right) .
$$

For 0 -cells $\vec{x}, \vec{y}$, we define

$$
\begin{aligned}
b_{\vec{x}, \vec{y}}(-,-) & :=\xi_{x}\left(-,-, x_{-}\right), \\
\sharp \vec{x}, \vec{y}(-) & :=\xi_{y}\left(y, 1_{y},-\right) .
\end{aligned}
$$

Then we have

$$
\begin{aligned}
\sharp_{\vec{x}, \vec{y}}\left(\mathfrak{d}_{\vec{x}, \vec{y}}(-)\right) & =\xi_{y}\left(y, 1_{y}, x_{-} \cdot-\right) \\
& =\xi_{y} \circ\left((y \downarrow \mathcal{C}) \times A_{-}\right)\left(y, 1_{y}, x_{-}\right) \\
& =\xi_{x} \circ\left(-^{*} \times A_{x}\right)\left(y, 1_{y}, x_{-}\right) \\
& =\xi_{x}\left(y,-, x_{-}\right) \\
& =b_{\vec{x}, \vec{y}}(y,-)
\end{aligned}
$$

where the third equation is by dinaturality of $\xi$.

$(\boldsymbol{n}+\mathbf{1})$-Cells for $(\boldsymbol{n}+\mathbf{1})$ odd. Given a $(n+1)$-cell $\vec{\varphi}=\left(\varphi, \varphi_{-}\right): \vec{\alpha} \rightarrow \vec{\beta}$, we define $F(\vec{\varphi}): F(\vec{\alpha}) \rightarrow F(\vec{\beta})$ as the following composite

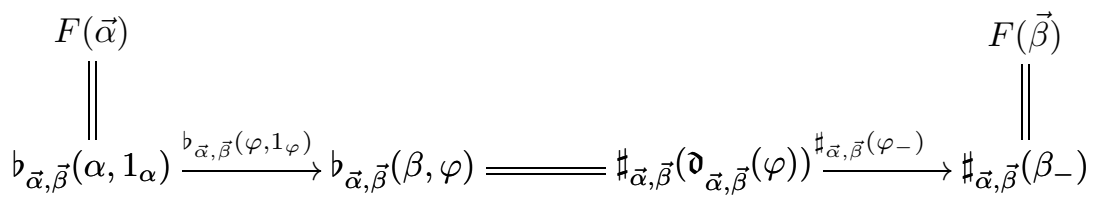

I.e.,

$$
F(\vec{\varphi}):=\sharp_{\vec{\alpha}, \vec{\beta}}\left(\varphi_{-}\right) *_{n} b_{\vec{\alpha}, \vec{\beta}}\left(\varphi, 1_{\varphi}\right) .
$$

Now, given parallel $(n+1)$-cells $\vec{\varphi}, \vec{\psi}: \vec{\alpha} \rightarrow \vec{\beta}$ we define

$$
b_{\vec{\varphi}, \vec{\psi}}(-,-):=\sharp_{\vec{\alpha}, \vec{\beta}}\left(\psi_{-}\right) *_{n} b_{\vec{\alpha}, \vec{\beta}}(-,-) \sharp_{\vec{\varphi}, \vec{\psi}}(-):=\sharp_{\vec{\alpha}, \vec{\beta}}(-) *_{n} b_{\vec{\alpha}, \vec{\beta}}\left(\varphi, 1_{\varphi}\right) .
$$

With this definition, we observe that

$$
\begin{aligned}
\sharp_{\vec{\varphi}, \vec{\psi}}\left(\mathfrak{d}_{\vec{\varphi}, \vec{\psi}}(-)\right) & =\sharp_{\vec{\varphi}, \vec{\psi}}\left(\psi_{-} *_{n} \mathfrak{d}_{\vec{\alpha}, \vec{\beta}}(-)\right) \\
& =\sharp_{\vec{\alpha}, \vec{\beta}}\left(\psi_{-} *_{n} \mathfrak{d}_{\vec{\alpha}, \vec{\beta}}(-)\right) *_{n} b_{\vec{\alpha}, \vec{\beta}}\left(\varphi, 1_{\varphi}\right) \\
& =\sharp_{\vec{\alpha}, \vec{\beta}}\left(\psi_{-}\right) *_{n} b_{\vec{\alpha}, \vec{\beta}}(\beta,-) *_{n} b_{\vec{\alpha}, \vec{\beta}}\left(\varphi, 1_{\varphi}\right) \\
& =\sharp_{\vec{\alpha}, \vec{\beta}}\left(\psi_{-}\right) *_{n} b_{\vec{\alpha}, \vec{\beta}}(\varphi,-) \\
& =b_{\vec{\alpha}, \vec{\beta}}(\varphi,-),
\end{aligned}
$$

where the third equation is by functoriality and the corresponding equation for $\sharp_{\vec{\alpha}, \vec{\beta}}(-)$. 
$(\boldsymbol{n}+\mathbf{1})$-Cells for $(\boldsymbol{n}+\mathbf{1})$ even. Given a $(n+1)$-cell $\vec{\varphi}: \vec{\alpha} \rightarrow \vec{\beta}$ we define $F(\vec{\varphi})$ to be the composite

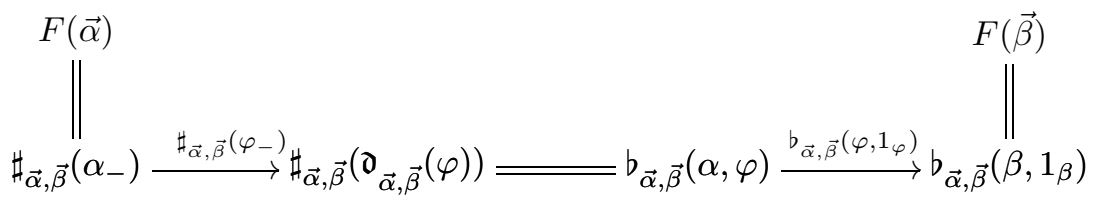

I.e.,

$$
F(\vec{\varphi}):=b_{\vec{\alpha}, \vec{\beta}}\left(\varphi, 1_{\varphi}\right) *_{n} \sharp_{\vec{\alpha}, \vec{\beta}}\left(\varphi_{-}\right) .
$$

Finally, for parallel $(n+1)$-cells $\vec{\varphi}, \vec{\psi}: \vec{\alpha} \rightarrow \vec{\beta}$ we define

$$
\begin{aligned}
b_{\vec{\varphi}, \vec{\psi}}(-,-) & :=b_{\vec{\alpha}, \vec{\beta}}(-,-) *_{n} \sharp_{\vec{\alpha}, \vec{\beta}}\left(\varphi_{-}\right), \\
\sharp_{\vec{\varphi}, \vec{\psi}}(-) & :=b_{\vec{\alpha}, \vec{\beta}}\left(\psi, 1_{\psi}\right) *_{n} \sharp_{\vec{\alpha}, \vec{\beta}}(-) .
\end{aligned}
$$

The verification that the corresponding equation holds is by an argument dual to the one for the case of $(n+1)$ odd given above.

Next, we will prove inductively that $F$ makes the diagram (B.7) commute. The key point which will allow us to prove this fact is that the action of the functor $F$ has a simplified description for those cells of $\int A$ in the image of $\iota_{x}$. (To prove the uniqueness of $F$ we will in fact show that all cells of $\int A$ occur as composites of cells in the images of the $\iota_{x}$, but more on this later.) In order to explain this it will be convenient to again introduce additional auxiliary operations $\diamond_{\vec{\alpha}, \vec{\beta}}(\vec{\varphi})$ and $\mathbf{\beta}_{\vec{\alpha}, \vec{\beta}}(\vec{\varphi})$ at each stage of the proof which are indexed by parallel $n$-cells $\vec{\alpha}, \vec{\beta}$ of $(x \downarrow \mathcal{C}) \times A_{x}$ and which act on $m$-cells $\vec{\varphi}$ of $(x \downarrow \mathcal{C}) \times A_{x}$ for $m \geq n$. We will also prove that these auxiliary functions interact in an appropriate way with each other and with $\sharp_{\iota_{x}(\vec{\alpha}), \iota_{x}(\vec{\beta})}(-)$ and $b_{\iota_{x}(\vec{\alpha}), \iota_{x}(\vec{\beta})}(-,-)$. These matters are described below in the proof.

0-Cells. Given $\vec{y}=\left(y, y_{-}, y_{o}\right)$ in $(x \downarrow \mathcal{C}) \times A_{x}$, we have

$$
F \circ \iota_{x}(\vec{y})=F\left(y, y_{o} \cdot y_{-}\right)=\xi_{y}\left(y, y, y_{o} \cdot y_{-}\right)=\xi_{x}\left(y, y_{-}, y_{o}\right),
$$

where the final equation is by dinaturality of the $\xi$.

Given two 0-cells $\vec{y}$ and $\vec{z}$, and a $n$-cell $\vec{\varphi}$ bounded by $\vec{y}$ and $\vec{z}$, respectively, we define

$$
\begin{aligned}
& \boldsymbol{\beta}_{\vec{y}, \vec{z}}(\vec{\varphi}):=\xi_{x}\left(z, \varphi_{-}, y_{o}\right) \text {, } \\
& \diamond_{\vec{y}, \vec{z}}(\vec{\varphi}):=\xi_{x}\left(\alpha, \alpha *_{0} y_{-}, y_{o}\right),
\end{aligned}
$$

and we observe that

$$
\begin{aligned}
\sharp_{\iota_{x}(\vec{y}), \iota_{x}(\vec{z})}\left(\iota_{x}(\vec{\varphi})_{-}\right) & =\xi_{x}\left(z, z_{-}, \varphi_{o}\right) *_{0} \boldsymbol{\phi}_{\vec{y}, \vec{z}}(\vec{\varphi}), \\
b_{\iota_{x}(\vec{y}), \iota_{x}(\vec{z})}(\varphi, \varphi) & =\diamond_{\vec{y}, \vec{z}}(\vec{\varphi}) .
\end{aligned}
$$

To see that these equations hold we reason as follows:

$$
\begin{aligned}
\sharp_{\iota_{x}(\vec{y}), \iota_{x}(\vec{z})}\left(\iota_{x}(\vec{\varphi})_{-}\right) & =\xi_{z}\left(z, z,\left(\varphi_{o} \cdot z_{-}\right) *_{0}\left(y_{o} \cdot \varphi_{-}\right)\right) \\
& =\xi_{z}\left(z, z, \varphi_{o} \cdot z_{-}\right) *_{0} \xi_{z}\left(z, z, y_{o} \cdot \varphi_{-}\right) \\
& =\xi_{x}\left(z, z_{-}, \varphi_{o}\right) *_{0} \xi_{x}\left(z, \varphi_{-}, y_{o}\right),
\end{aligned}
$$


where the final equation is by dinaturality of $\xi$, and similarly

$$
\begin{aligned}
b_{\iota_{x}(\vec{y}), \iota_{x}(\vec{z})}(\varphi, \varphi) & =\xi_{y}\left(\varphi, \varphi, y_{o} \cdot y_{-}\right) \\
& =\xi_{x}\left(\varphi, \varphi *_{0} y_{-}, y_{o}\right)
\end{aligned}
$$

where the final equation is by dinaturality of $\xi$.

We also note that, by definition,

$$
\boldsymbol{\beta}_{\vec{y}, \vec{z}}(\vec{\varphi}) *_{0} \diamond_{\vec{y}, \vec{z}}(\vec{\varphi})=\xi_{x}\left(f, f_{-}, y_{o}\right)
$$

1-Cells. Given $\vec{f}=\left(f, f_{-}, f_{o}\right): \vec{y} \rightarrow \vec{z}$ in $(x \downarrow \mathcal{C}) \times A_{x}$, we have

$$
\begin{aligned}
F \circ \iota_{x}(\vec{f}) & =\sharp_{\iota_{x}(\vec{y}), \iota_{x}(\vec{z})}\left(\iota_{x}(\vec{f})_{-}\right) *_{0} b_{\iota_{x}(\vec{y}), \iota_{x}(\vec{z})}(f, f) \\
& =\xi_{x}\left(z, z_{-}, f_{o}\right) *_{0} \boldsymbol{\beta}_{\vec{y}, \vec{z}}(\vec{f}) *_{0} \diamond_{\vec{y}, \vec{z}}(\vec{f}) \\
& =\xi_{x}\left(z, z_{-}, f_{o}\right) *_{0} \xi_{x}\left(f, f_{-}, y_{o}\right) \\
& =\xi_{x}\left(f, f_{-}, f_{o}\right) .
\end{aligned}
$$

where the second equation is by (B.8) and (B.9).

Now, given parallel 1-cells $\vec{f}, \vec{g}: \vec{y} \rightarrow \vec{z}$, we define

$$
\begin{aligned}
& \boldsymbol{\boldsymbol { \beta }}_{\vec{f}, \vec{g}}(\vec{\varphi}):=\boldsymbol{\alpha}_{\vec{y}, \vec{z}}(\vec{g}) *_{0} \diamond_{\vec{y}, \vec{z}}(\vec{\varphi}), \\
& \diamond_{\vec{f}, \vec{g}}(\vec{\varphi}):=\boldsymbol{\aleph}_{\vec{y}, \vec{z}}(\vec{\varphi}) *_{0} \diamond_{\vec{y}, \vec{z}}(\vec{f}),
\end{aligned}
$$

and we observe that

$$
\begin{aligned}
\sharp_{\iota_{x}(\vec{f}), \iota_{x}(\vec{g})}\left(\iota_{x}(\vec{\varphi})_{-}\right) & =\xi_{x}\left(z, z_{-}, \varphi_{o}\right) *_{0} \diamond_{\vec{f}, \vec{g}}(\vec{\varphi}), \\
b_{\iota_{x}(\vec{f}), \iota_{x}(\vec{g})}(\varphi, \varphi) & =\xi_{x}\left(z, z_{-}, g_{o}\right) *_{0} \boldsymbol{\leftrightarrow}_{\vec{f}, \vec{g}}(\vec{\varphi}) .
\end{aligned}
$$

The proof that these equations hold is immediate from the definition together with (B.8) and (B.9). Similarly, we also have that

$$
\boldsymbol{\beta}_{\vec{f}, \vec{g}}(\vec{\varphi}) *_{1} \diamond_{\vec{f}, \vec{g}}(\vec{\varphi})=\xi_{x}\left(\varphi, \varphi_{-}, y_{o}\right) \text {. }
$$

2-Cells. Given $\vec{\alpha}=\left(\alpha, \alpha_{-}, \alpha_{o}\right): \vec{f} \rightarrow \vec{g}$ in $(x \downarrow \mathcal{C}) \times A_{x}$ we have

$$
\begin{aligned}
F \circ \iota_{x}(\vec{\alpha}) & =b_{\iota_{x}(\vec{f}), \iota_{x}(\vec{g})}(\alpha, \alpha) *_{1} \sharp_{\iota_{x}(\vec{f}), \iota_{x}(\vec{g})}\left(\iota_{x}(\vec{\alpha})_{-}\right) \\
& =\left(\xi_{x}\left(z, z_{-}, g_{o}\right) *_{0} \boldsymbol{\beta}_{\vec{f}, \vec{g}}(\vec{\alpha})\right) *_{1}\left(\xi_{x}\left(z, z_{-}, \alpha_{o}\right) *_{0} \diamond_{\vec{f}, \vec{g}}(\vec{\alpha})\right) \\
& =\xi_{x}\left(z, z_{-}, \alpha_{o}\right) *_{0}\left(\boldsymbol{\beta}_{\vec{f}, \vec{g}}(\vec{\alpha}) *_{1} \boldsymbol{中}_{\vec{f}, \vec{g}}(\vec{\alpha})\right) \\
& =\xi_{x}\left(z, z_{-}, \alpha_{o}\right) *_{0} \xi_{x}\left(\alpha, \alpha_{-}, y_{o}\right) \\
& =\xi_{x}(\vec{\alpha}),
\end{aligned}
$$

where the first equation is (B.10) and (B.11). 
This calculation can be visualized as follows:

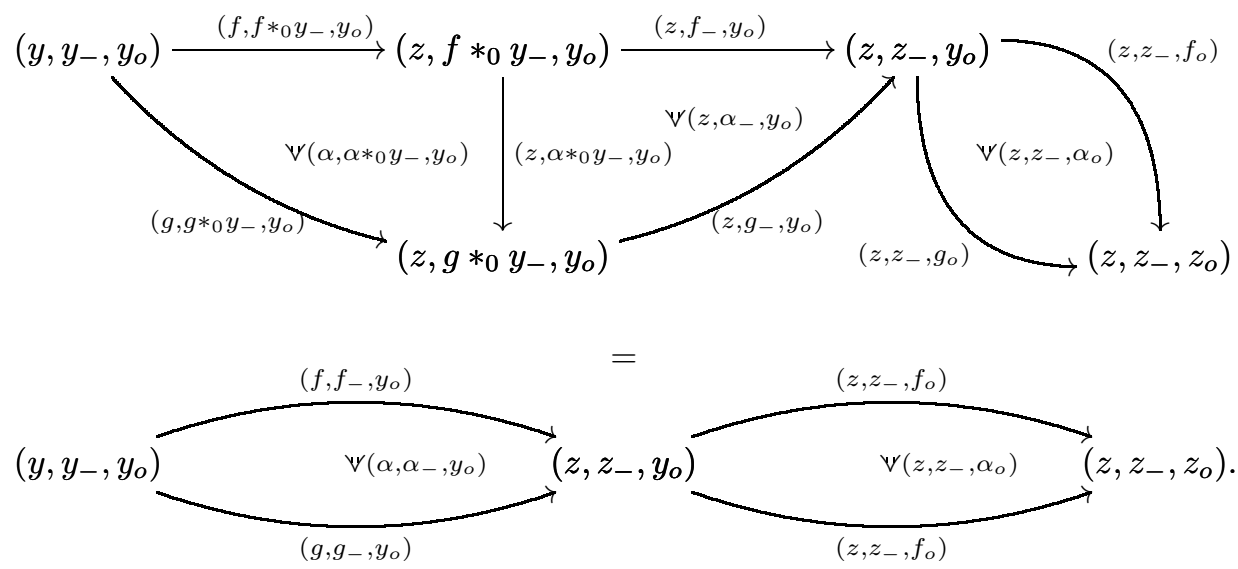

Now, given parallel 2-cells $\vec{\alpha}, \vec{\beta}: \vec{f} \rightarrow \vec{g}$ we define

$$
\begin{aligned}
& \boldsymbol{\leftrightarrow}_{\vec{\alpha}, \vec{\beta}}(\vec{\varphi}):=\boldsymbol{\alpha}_{\vec{f}, \vec{g}}(\vec{\beta}) *_{1} \diamond_{\vec{f}, \vec{g}}(\vec{\varphi}), \\
& \diamond_{\vec{\alpha}, \vec{\beta}}(\vec{\varphi}):=\boldsymbol{\leftrightarrow}_{\vec{f}, \vec{g}}(\vec{\varphi}) *_{1} \diamond_{\vec{f}, \vec{g}}(\vec{\alpha}) .
\end{aligned}
$$

It then follows that

$$
\begin{aligned}
b_{\iota_{x}(\vec{\alpha}), \iota_{x}(\vec{\beta})}(\varphi, \varphi) & =\xi_{x}\left(z, z_{-}, \alpha_{o}\right) *_{0} \diamond_{\vec{\alpha}, \vec{\beta}}(\vec{\varphi}), \\
\sharp_{\iota_{x}(\vec{\alpha}), \iota_{x}(\vec{\beta})}\left(\iota_{x}(\vec{\varphi})_{-}\right) & =\xi_{x}\left(z, z_{-}, \varphi_{o}\right) *_{0} \boldsymbol{\leftrightarrow}_{\vec{\alpha}, \vec{\beta}}(\vec{\varphi}) .
\end{aligned}
$$

and that

$$
\hat{\beta}_{\vec{\alpha}, \vec{\beta}}(\vec{\varphi}) *_{2} \diamond_{\vec{\alpha}, \vec{\beta}}(\vec{\varphi})=\xi_{x}\left(\varphi, \varphi_{-}, y_{o}\right) .
$$

All three of these equations are easily verified using the corresponding equations for $\boldsymbol{\beta}_{\vec{f}, \vec{g}}$ and $\diamond_{\vec{f}, \vec{g}}$.

We now describe the general structure of the inductive proof. At each stage $(n+1)$ we will prove that $F \circ \iota_{x}(\vec{\varphi})=\xi_{x}(\vec{\varphi})$, for $\vec{\varphi}$ a $(n+1)$-cell. We will also define, for parallel $(n+1)$-cells $\vec{\varphi}, \vec{\psi}: \vec{\alpha} \rightarrow \vec{\beta}$, Next, given parallel $(n+1)$-cells $\vec{\varphi}, \vec{\psi}: \vec{\alpha} \rightarrow \vec{\beta}$ we define

$$
\boldsymbol{\alpha}_{\vec{\varphi}, \vec{\psi}}(\vec{\gamma}):=\boldsymbol{\leftrightarrow}_{\vec{\alpha}, \vec{\beta}}(\vec{\psi}) *_{n} \diamond_{\vec{\alpha}, \vec{\beta}}(\vec{\gamma}) \diamond_{\vec{\varphi}, \vec{\psi}}(\vec{\gamma}):=\boldsymbol{\alpha}_{\vec{\alpha}, \vec{\beta}}(\vec{\gamma}) *_{n} \diamond_{\vec{\alpha}, \vec{\beta}}(\vec{\varphi}),
$$

$\mathbf{p}_{\vec{\varphi}, \vec{\psi}}$ and we will prove the corresponding equations:

$$
b_{\iota_{x}(\vec{\varphi}), \iota_{x}(\vec{\psi})}(\gamma, \gamma)= \begin{cases}\xi_{x}\left(z, z_{-}, \varphi_{o}\right) *_{0} \diamond_{\vec{\varphi}, \vec{\psi}}(\vec{\gamma}) & \text { for }(n+1) \text { even, } \\ \xi_{x}\left(z, z_{-}, \psi_{o}\right) *_{0} \boldsymbol{\phi}_{\vec{\varphi}, \vec{\psi}}(\vec{\gamma}) & \text { for }(n+1) \text { odd }\end{cases}
$$

and

$$
\sharp_{\iota_{x}(\vec{\varphi}), \iota_{x}(\vec{\psi})}\left(\iota_{x}(\vec{\gamma})_{-}\right)=\left\{\begin{array}{l}
\xi_{x}\left(z, z_{-}, \gamma_{o}\right) *_{0} \boldsymbol{\beta}_{\vec{\varphi}, \vec{\psi}}(\vec{\gamma}) \text { for }(n+1) \text { even, } \\
\xi_{x}\left(z, z_{-}, \gamma_{o}\right) *_{0} \diamond_{\vec{\varphi}, \vec{\psi}}(\vec{\gamma}) \text { for }(n+1) \text { odd. }
\end{array}\right.
$$

Finally, we will show at each stage that

$$
\boldsymbol{\beta}_{\vec{\varphi}, \vec{\psi}}(\vec{\gamma}) *(n+1) \diamond_{\vec{\varphi}, \vec{\psi}}(\vec{\gamma})=\xi_{x}\left(\gamma, \gamma_{-}, y_{o}\right) \text {. }
$$

We now turn to the proof. 
$(\boldsymbol{n}+\mathbf{1})$-Cells for $(\boldsymbol{n}+\mathbf{1})$ odd. Given a $(n+1)$-cell $\vec{\varphi}: \vec{\alpha} \rightarrow \vec{\beta}$ we have

$$
\begin{aligned}
F \circ \iota_{x}(\vec{\varphi}) & =\sharp_{\iota_{x}(\vec{\alpha}) \iota_{x}(\vec{\beta})}\left(\iota_{x}(\vec{\varphi})_{-}\right) *_{n} b_{\iota_{x}(\vec{\alpha}) \iota_{x}(\vec{\beta})}(\varphi, \varphi) \\
& =\left(\xi_{x}\left(z, z_{-}, \varphi_{o}\right) *_{0} \boldsymbol{\leftrightarrow}_{\vec{\alpha}, \vec{\beta}}(\vec{\varphi})\right) *_{n}\left(\xi_{x}\left(z, z_{-}, \alpha_{o}\right) *_{0} \diamond_{\vec{\alpha}, \vec{\beta}}(\vec{\varphi})\right) \\
& =\xi_{x}\left(z, z_{-}, \varphi_{o}\right) *_{0}\left(\boldsymbol{\leftrightarrow}_{\vec{\alpha}, \vec{\beta}}(\vec{\varphi}) *_{n} \diamond_{\vec{\alpha}, \vec{\beta}}(\vec{\varphi})\right) \\
& =\xi_{x}(\vec{\varphi}) .
\end{aligned}
$$

Next, given parallel $(n+1)$-cells $\vec{\varphi}, \vec{\psi}: \vec{\alpha} \rightarrow \vec{\beta}$, we prove that the required equations hold as follows:

$$
\begin{aligned}
b_{\iota_{x}(\vec{\varphi}), \iota_{x}(\vec{\psi})}(\gamma, \gamma) & =\sharp_{\iota_{x}(\vec{\alpha}), \iota_{x}(\vec{\beta})}\left(\iota_{x}(\vec{\psi})_{-}\right) *_{n} b_{\iota_{x}(\vec{\alpha}), \iota_{x}(\vec{\beta})}(\gamma, \gamma) \\
& =\left(\xi_{x}\left(z, z_{-}, \gamma_{o}\right) *_{0} \boldsymbol{\beta}_{\vec{\alpha}, \vec{\beta}}(\vec{\gamma})\right) *_{n}\left(\xi_{x}\left(z, z_{-}, \varphi_{o}\right) *_{0} \diamond_{\vec{\alpha}, \vec{\beta}}(\vec{\gamma})\right) \\
& =\xi_{x}\left(z, z_{-}, \gamma_{o}\right) *_{0}\left(\boldsymbol{\beta}_{\vec{\alpha}, \vec{\beta}}(\vec{\gamma}) *_{n} \diamond_{\vec{\alpha}, \vec{\beta}}(\vec{\gamma})\right) \\
& =\xi_{x}(\vec{\gamma}),
\end{aligned}
$$

where we have used the equations given by the induction hypothesis. Similarly, we see that

$$
\boldsymbol{\beta}_{\vec{\varphi}, \vec{\psi}}(\vec{\gamma}) *_{n+1} \diamond_{\vec{\varphi}, \vec{\psi}}(\vec{\gamma})=\xi_{x}\left(\gamma, \gamma_{-}, y_{o}\right) \text {. }
$$

$(n+1)$-Cells for $(n+1)$ even. The calculations here are dual (in the appropriate sense) to those for the odd case and are left to the reader.

This completes the proof of the following lemma:

Lemma B.1. With the definition of $F$ given above, (B.7) commutes.

Finally, in order to show that $F$ is the canonical functor $\int A \rightarrow \mathcal{A}$ making (B.7) commute, we will prove that every cell of $\int A$ can be written as a composite of cells in the image of the maps $\iota_{x}$. The construction of this decomposition of the cells of $\int A$, which is again by induction on dimension, involves constructing auxiliary operations (which mimic $b$ and $\sharp$ ) and then proving that these satisfy suitable equations.

0-Cells. Given a 0 -cell $\vec{x}=\left(x, x_{-}\right)$in $\int A$ we have that

$$
\vec{x}=\iota_{x}\left(x, x, x_{-}\right) \text {. }
$$

Given 0 -cells $\vec{x}$ and $\vec{y}$ and a $(n+1)$-cell $\vec{\varphi}$ bounded by $\vec{x}$ and $\vec{y}$ in $\int A$, we define

$$
\begin{aligned}
& \hat{b}_{\vec{x}, \vec{y}}(\vec{\varphi}):=\iota_{x}\left(\varphi, \varphi, x_{-}\right), \\
& \hat{\sharp}_{\vec{x}, \vec{y}}(\vec{\varphi}):=\iota_{y}\left(y, y, \varphi_{-}\right) .
\end{aligned}
$$

We observe that we have the equations

$$
\begin{aligned}
& \hat{b}_{\vec{x}, \vec{y}}(\vec{\varphi})=\left(\varphi, \mathfrak{d}_{\vec{x}, \vec{y}}(\varphi)\right), \\
& \hat{\sharp}_{\vec{x}, \vec{y}}(\vec{\varphi})=\left(y, \varphi_{-}\right),
\end{aligned}
$$

since

$$
\iota_{x}\left(-,-, x_{-}\right)_{-}=\left(x_{-} \cdot-\right) *_{0}\left(x_{-} \cdot-\right)=x_{-} \cdot-=\mathfrak{d}_{\vec{x}, \vec{y}}(-),
$$

and the first equation is trivial by definition of $\iota_{y}(y, y,-)$.

1-Cells. Given $\vec{f}=\left(f, f_{-}\right): \vec{x} \rightarrow \vec{y}$ we have

$$
\iota_{y}\left(y, y, f_{-}\right) *_{0} \iota_{x}\left(f, f, x_{-}\right)=\vec{f} .
$$

by the equations above (since $\mathfrak{d}_{\vec{x}, \vec{y}}(f)$ is the domain of $f_{-}$). 
Next, given parallel $\vec{f}, \vec{g}: \vec{x} \rightarrow \vec{y}$ in $\int A$, we define

$$
\begin{aligned}
& \hat{b}_{\vec{f}, \vec{g}}(\vec{\varphi}):=\hat{\sharp}_{\vec{x}, \vec{y}}(\vec{g}) *_{0} \hat{b}_{\vec{x}, \vec{y}}(\vec{\varphi}), \\
& \hat{\sharp}_{\vec{f}, \vec{g}}(\vec{\varphi}):=\hat{\sharp}_{\vec{x}, \vec{y}}(\vec{\varphi}) *_{0} \hat{b}_{\vec{x}, \vec{y}}(\vec{f}) .
\end{aligned}
$$

Now, we also have the equations

$$
\begin{aligned}
& \hat{b}_{\vec{f}, \vec{g}}(\vec{\varphi})=\left(\varphi, \mathfrak{d}_{\vec{f}, \vec{g}}(\varphi)\right), \\
& \hat{\sharp}_{\vec{f}, \vec{g}}(\vec{\varphi})=\left(f, \varphi_{-}\right),
\end{aligned}
$$

as the reader may readily verify.

We may now describe the general inductive construction. Explicitly, for parallel $(n+1)$-cells $\vec{\varphi}, \vec{\psi}: \vec{\alpha} \rightarrow \vec{\beta}$, we define

$$
\hat{b}_{\vec{\varphi}, \vec{\psi}}(\vec{\gamma}):= \begin{cases}\hat{\sharp}_{\vec{\alpha}, \vec{\beta}}(\vec{\psi}) *_{n} \hat{b}_{\vec{\alpha}, \vec{\beta}}(\vec{\gamma}) & \text { for }(n+1) \text { odd, } \\ \hat{b}_{\vec{\alpha}, \vec{\beta}}(\vec{\gamma}) *_{n} \hat{\sharp}_{\vec{\alpha}, \vec{\beta}}(\vec{\varphi}) & \text { for }(n+1) \text { even, }\end{cases}
$$

and

$$
\hat{\sharp}_{\vec{\varphi}, \vec{\psi}}(\vec{\gamma}):= \begin{cases}\hat{\sharp}_{\vec{\alpha}, \vec{\beta}}(\vec{\gamma}) *_{n} \hat{b}_{\vec{\alpha}, \vec{\beta}}(\vec{\varphi}) & \text { for }(n+1) \text { odd, } \\ \hat{b}_{\vec{\alpha}, \vec{\beta}}(\vec{\psi}) *_{n} \hat{\sharp}_{\vec{\alpha}, \vec{\beta}}(\vec{\gamma}) & \text { for }(n+1) \text { even. }\end{cases}
$$

We note that with these definitions one easily proves by induction that the equations

$$
\begin{aligned}
& \hat{b}_{\vec{\varphi}, \vec{\psi}}(\vec{\gamma})=\left(\gamma, \mathfrak{d}_{\vec{\varphi}, \vec{\psi}}(\gamma)\right), \\
& \hat{\sharp}_{\vec{\varphi}, \vec{\psi}}(\vec{\gamma})=\left(\varphi, \gamma_{-}\right)
\end{aligned}
$$

hold. So, if $\vec{\gamma}: \vec{\varphi} \rightarrow \vec{\psi}$ is any $(n+1)$-cell of $\int A$, then

$$
\vec{\gamma}=\hat{\sharp}_{\vec{\varphi}, \vec{\psi}}(\vec{\gamma}) *_{n} \hat{b}_{\vec{\varphi}, \vec{\psi}}(\vec{\gamma}) \text {. }
$$

Therefore, since each cell of the form $\hat{b}_{\vec{\varphi}, \vec{\psi}}(\vec{\gamma})$ or $\hat{\sharp}_{\vec{\varphi}, \vec{\psi}}(\vec{\gamma})$ is by construction a composite of cells in the image of the $\iota$ it follows that every cell of $\int A$ can be represented as a composite of cells in the image of the $\iota$ and therefore $F$ is the canonical map $\int A \rightarrow \mathcal{A}$ making (B.7) commute. This completes the proof of the following:

Theorem B.2. Given a functor $A: \mathcal{C} \rightarrow \omega$-Cat of $\omega$-categories, the Grothendieck construction $\int A$ described in Section 3 is the coend $\int^{x}(x \downarrow \mathcal{C}) \times A_{x}$.

\section{References}

1. S. Awodey, P. Hofstra, and M. A. Warren, Martin-Löf complexes, submitted, available at arXiv:0906.4521.

2. S. Awodey and M. A. Warren, Homotopy theoretic models of identity types, Math. Proc. Cambridge Philos. Soc. 146 (2009), no. 1, 45-55.

3. M. A. Batanin, Monoidal globular categories as a natural environment for the theory of weak n-categories, Adv. Math. 136 (1998), no. 1, 39-103.

4. P. Dybjer, Internal type theory, Types for Proofs and Programs (Torino, 1995) (Berardi Sefano and M. Coppo, eds.), Lecture Notes in Comput. Sci., vol. 1158, Springer, Berlin, 1996, pp. 120134 .

5. N. Gambino and R. Garner, The identity type weak factorisation system, Theoret. Comput. Sci. 409 (2008), no. 1, 94-109.

6. R. Garner, Two-dimensional models of type theory, Math. Structures Comput. Sci. 19 (2009), no. 4, 687-736.

7. C. Hermida, Some properties of Fib as a fibred 2-category, J. Pure Appl. Algebra 134 (1999), no. 1, 83-109. 
8. M. Hofmann, Syntax and semantics of dependent types, Semantics and Logics of Computation (Cambridge, 1995) (A. M. Pitts and P. Dybjer, eds.), Publ. Newton Inst., vol. 14, Cambridge Univ. Press, Cambridge, 1997, pp. 79-130.

9. M. Hofmann and T. Streicher, The groupoid interpretation of type theory, Twenty-Five Years of Constructive Type Theory (Venice, 1995) (G. Sambin and J. M. Smith, eds.), Oxford Logic Guides, vol. 36, Oxford Univ. Press, New York, 1998, pp. 83-111.

10. B. Jacobs, Categorical logic and type theory, Stud. Logic Found. Math., vol. 141, NorthHolland, Amsterdam, 1999.

11. M. M. Kapranov and V. A. Voevodsky, $\infty$-groupoids and homotopy types, Cahiers Topologie Géom. Différentielle Catég. 32 (1991), no. 1, 29-46.

12. Y. Lafont, F. Métayer, and K. Worytkiewicz, A folk model structure on omega-cat, Adv. Math. 224 (2010), no. 3, 1183-1231.

13. F. W. Lawvere, Adjointness in foundations, Repr. Theory Appl. Categ. 16 (2006), 1-16. Reprinted from Dialectica 23 (1969).

14. T. Leinster, Higher operads, higher categories, London Math. Soc. Lecture Note Ser., vol. 298, Cambridge Univ. Press, Cambridge, 2004.

15. P. L. Lumsdaine, Weak $\omega$-categories from intensional type theory, Typed Lambda Calculi and Applications (Brasília, 2009) (P.-L. Curien, ed.), Lecture Notes in Comput. Sci., vol. 5608, Springer, Berlin, 2009, pp. 172-187.

16. P. Martin-Löf, An intuitionistic theory of types: predicative part, Logic Colloquium '73 (Bristol, 1973) (H. E. Rose and J. C. Shepherdson, eds.), Stud. Logic Found. Math., vol. 80, North-Holland, Amsterdam, 1975, pp. 73-118.

17. F. Métayer, Resolutions by polygraphs, Theory Appl. Categ. 11 (2003), no. 7, 148-184.

18. I. Moerdijk and J.-A. Svensson, Algebraic classification of equivariant homotopy 2-types. I, J. Pure Appl. Algebra 89 (1993), no. 1-2, 187-216.

19. B. Nordström, K. Petersson, and J. M. Smith, Programming in Martin-Löf's type theory: An introduction, Internat. Ser. Monogr. Comput. Sci., vol. 7, Oxford Univ. Press, New York, 1990.

20. T. Palm, Categories with slicing, Theory Appl. Categ. 22 (2009), no. 5, 97-135.

21. R. Street, The algebra of oriented simplexes, J. Pure Appl. Algebra 49 (1987), no. 3, 283-335.

22. The petit topos of globular sets, J. Pure Appl. Algebra 154 (2000), no. 1-3, 299-315.

23. T. Streicher, Semantics of type theory, with a foreword by M. Wirsing, Progr. Theoret. Comput. Sci., Birkhäuser, Boston, MA, 1991.

24. _ Investigations into intensional type theory, Habilitationsschrift, Ludwig-MaximiliansUniversität München, 1993.

25. B. van den Berg and R. Garner, Types are weak $\omega$-groupoids, submitted, available at arXiv: 0812.0298.

26. D. Verity, Complicial sets characterising the simplicial nerves of strict $\omega$-categories, Mem. Amer. Math. Soc. 193 (2008), no. 905.

27. M. A. Warren, Homotopy theoretic aspects of constructive type theory, Ph.D. Thesis, Carnegie Mellon University, 2008.

28. A characterization of representable intervals, submitted, available at arXiv:0903. 3743.

Department of Mathematics and Statistics, University of Ottawa, 585 King Edward Ave., Ottawa, ON K1N 6N5, Canada

E-mail address: mwarren@uottawa.ca 